1995

\title{
Characterization of composite materials from temporal thermal response
}

Pierre Richard Emeric

College of William \& Mary - Arts \& Sciences

Follow this and additional works at: https://scholarworks.wm.edu/etd

Part of the Materials Science and Engineering Commons

\section{Recommended Citation}

Emeric, Pierre Richard, "Characterization of composite materials from temporal thermal response" (1995). Dissertations, Theses, and Masters Projects. Paper 1539623868.

https://dx.doi.org/doi:10.21220/s2-e3b0-ps87

This Dissertation is brought to you for free and open access by the Theses, Dissertations, \& Master Projects at W\&M ScholarWorks. It has been accepted for inclusion in Dissertations, Theses, and Masters Projects by an authorized administrator of W\&M ScholarWorks. For more information, please contact scholarworks@wm.edu. 


\section{INFORMATION TO USERS}

This manuscript has been reproduced from the microfilm master. UMI films the text directly from the original or copy submitted. Thus, some thesis and dissertation copies are in typewriter face, while others may be from any type of computer printer.

The quality of this reproduction is dependent upon the quality of the copy submitted. Broken or indistinct print, colored or poor quality illustrations and photographs, print bleedthrough, substandard margins, and improper alignment can adversely affect reproduction.

In the unlikely event that the author did not send UMI a complete manuscript and there are missing pages, these will be noted. Also, if unauthorized copyright material had to be removed, a note will indicate the deletion.

Oversize materials (e.g., maps, drawings, charts) are reproduced by sectioning the original, beginning at the upper left-hand corner and continuing from left to right in equal sections with small overlaps. Each original is also photographed in one exposure and is included in reduced form at the back of the book.

Photographs included in the original manuscript have been reproduced . xerographically in this copy. Higher quality $6^{n} \times 9^{n}$ black and white photographic prints are available for any photographs or illustrations appearing in this copy for an additional charge. Contact UMI directly to order.

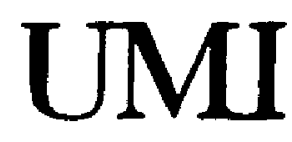

A Bell \& Howell Information Company 



\title{
CHARACTERIZATION OF COMPOSITE MATERIALS
}

FROM TEMPORAL THERMAL RESPONSE

\author{
A Dissertation \\ Presented to \\ The Faculty of the Applied Science Program \\ The College of William and Mary in Virginia
}

\author{
In Partial Fulfillment \\ Of the Requirements for the Degree of \\ Doctor of Philosophy
}

by

Pierre Emeric

1995 
UMI Number: 9537553

Copyright 1996 by

Emeric, Pierre Richard

All rights reserved.

UMI Microform 9537553

Copyright 1995, by UMI Company. All rights reserved.

This microform edition is protected against unauthorized copying under Title 17, Onited States code.

\section{UMI}

300 North Zeeb Road

Ann Arbor, MI 48103 


\section{APPROVAL SHEET}

This dissertation is submitted in partial fulfillment of

the requirements for the degree of

Doctor of Philosophy

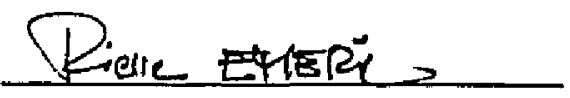

Pierre Emeric

Approved, May 1995

$$
\frac{\text { i.lliam R L } / 7 / 2}{\text { William P. Winfree, Ph.D. }}
$$

Adjunct Professor of Applied Science

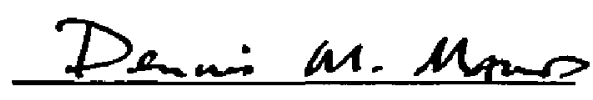

Dennis M. Manos, Ph.D.

CSX Professor of Applied Science and Physics

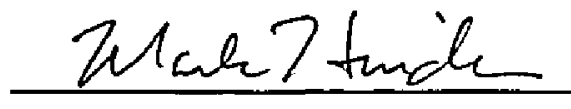

Mark K. Hinders, Ph.D.

Assistant Professor of Applied Science and Physics

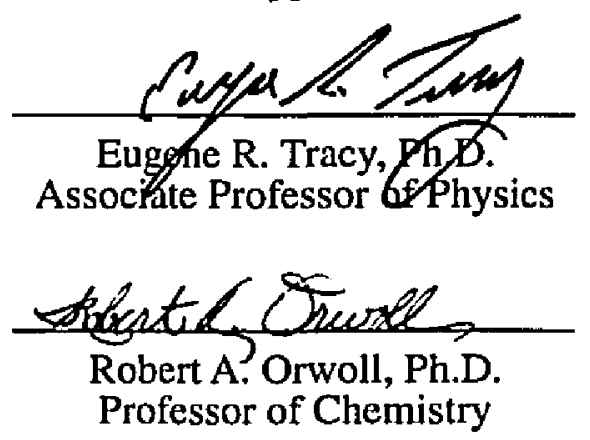


To the memory of my grandfather. 
TABLE OF CONTENTS

Page

ACKNOWLEDGEMENTS $\mathrm{v}$

LIST OF TABLES vi

LIST OF FIGURES $\quad$ vii

ABSTRACT

INTRODUCTION 2

$\begin{array}{lll}\text { CHAPTER I. HISTORICAL REVIEW } & 7\end{array}$

CHAPTER II. THEORETICAL MODELS FOR NEW MEASUREMENT

$\begin{array}{ll}\text { TECHNIQUE } & 18\end{array}$

CHAPTER III. EXPERIMENTAL PROCEDURES AND APPARATUS, SPECIMENS STUDIED $\quad 46$

CHAPTER IV. EXPERIMENTAL RESULTS $\quad 59$

CHAPTER V. QUANTITATIVE ANALYSIS AND INTERPRETATION

$\begin{array}{lr}\text { CONCLUSION } & 89\end{array}$

$\begin{array}{ll}\text { APPENDIX A } & 92\end{array}$

$\begin{array}{ll}\text { APPENDIX B } & 104\end{array}$

$\begin{array}{ll}\text { APPENDIX C } & 113\end{array}$

$\begin{array}{ll}\text { APPENDIX D } & 118\end{array}$

$\begin{array}{ll}\text { REFERENCES/ENDNOTES } & 131\end{array}$

$\begin{array}{ll}\text { BIBLIOGRAPHY } & 134\end{array}$ 


\section{ACKNOWLEDGEMENTS}

The writer wishes to thank Ray Parker and Dr. Meng-Chou Wu for their help and advice during the past two years. 


\section{LIST OF TABLES}

$\begin{array}{lll}\text { Table } & \text { Page }\end{array}$

2.1 Thermal properties for simulations in Fig. 2.3

2.2 Thermal properties for simulations in Fig. 2.4

2.3 Values for the parameters used in Fig. 2.9, 2.10, 2.11 and 2.12

5.1 Measurements of thermal diffusivity and conductivity for three layer specimens. 


\section{LIS'T OF FIGURES}

Figure

Page

1.1 Solid of volume V with outer surface S. 9

1.2 Principle of the steady-state longitudinal heat flow method. 13

$\begin{array}{lll}2.1 & \text { Model for a multilayer structure. } & 19\end{array}$

2.2 Interpretation of the analytical solution for $v_{3}$.

2.3 Simulated temperature profiles for two sets of parameters for a three layer structure with unit flash heating at $t=0$.

2.4 Simulated temperature profiles for one set of parameters for a two layer structure with unit constant heating starting at $t=0$.

2.5 Geometry for 2-D one fiber composite system and periodic heat source.

2.6 Point source at $S_{0}$ and expansion at point $P$.

2.7 Geometry and definitions used in simulations

2.8 Surface plot of the temperature distribution in the matrix due to the heat source.

2.9 Surface plot of the temperature distribution due to the scattering off a fiber less conductive/diffusive than the matrix.

2.10 Side view of the surface plot shown in Fig. 2.9

2.11 Surface plot of the temperature distribution due to the scattering off a fiber. 42

2.12 Side view of the temperature distribution shown in Fig. 2.11.

2.13 Simulations of the temperature profile as afunction of $y$ for fiber diameters 50,75 and $200 \mu \mathrm{m}$. 
Figure

2.14 Simulations of the temperature profile as a function of $y$ for depths $\mathrm{r}_{0}$ of 200,250 and $300 \mu \mathrm{m}$.

2.15 Simulations of the temperature profile as a function of depth for excitation frequencies ranging from 1 to $20 \mathrm{~Hz}$.

3.1 Optical schematic of infrared imager.

3.2 Experimental setup with laser and point infrared detector for data acquisition on two layer specimens.

3.3 Experimental setup with flash lamp and point infrared detector for data acquisition on three layer specimens.

3.4 Geometry of 3 layered specimen with simulated corrosion.

3.5 Geometry of 3 layered specimens with air and glycerin.

3.6 Principle of the experiment for characterization of composite materials.

3.7 Geometry of aluminum specimen.

3.8 Geometry of RBSN composite specimens.

3.9 Experimental setup for data acquisition on composite materials.

4.1 Example of raw data for temperature response of two layer specimens for an excitation frequency of $2 \mathrm{~Hz}$.

4.2 Example of normalized temperature response of two layer specimens for an excitation frequency of $2 \mathrm{~Hz}$.

4.3 Example of normalized temperature response of two layer specimens for an excitation frequency of $5 \mathrm{~Hz}$.

4.4 Thermal response of specimen 2 for an excitation frequency of $0.3 \mathrm{~Hz}$.

4.5 X-ray tomography and thermal images of simulated corrosion specimen.

4.6 Temperature profiles for simulated corrosion sample for two locations.

4.7 Infrared image of the specimen with glycerin inner layer with flash heating. 
Figure

Page

4.7 Infrared image of the specimen with glycerin inner layer with flash heating.

4.8 Temperature profiles of two experiments for specimen with glycerin inner layer.

4.9 Infrared image of the specimen with air inner layer with flash heating at the back.

4.10 Time-temperature profile for sample with epoxy inner layer with flash heat deposit.

4.11 Pixel by pixel Fourier transform for a multi-image.

4.12 Example of time and frequency domain contrast enhanced images of the temperature response of original sample RBSN1.

4.13 First harmonics of the frequency domain multi-image for RBSN1.

4.14 Frequency domain contrast enhanced images of the temperature response of degraded sample RBSN2.

4.15 First harmonics of the frequency domain multi-image for RBSN2.

4.16 First harmonic frequency domain image of aluminum specimen.

5.1 Estimated thermal diffusivity of coating as a function of frequency for two layer samples with varying coating thickness.

5.2 Estimated thermal conductivity of coating as a function of frequency for two layer samples with varying coating thickness.

5.3 a. Simulations of the temperature profiles in the fistt layer of two layer sample 1 after $0.1,1.0$, and 2.0 seconds.

b. Simulations of the temperature profiles in the fisrt layer of two layer sample 2 after $0.1,1.0$, and 2.0 seconds.

c. Simulations of the temperature profiles in the fisrt layer of two layer sample 3 after $0.1,1.0$, and 2.0 seconds.

5.4 Column average values of the third harmonic of th Fourier transform of the surface temperature for frabricated aluminum specimen with air hole. 
Figure

5.5 a. Column average for the $1^{\text {st }}$ harmonic freauency domain image of RBSN1. 85

b. Column average for the $3^{\text {rd }}$ harmonic freauency domain image of RBSN1. 85

c. Column average for the $5^{\text {th }}$ harmonic freauency domain image of RBSN1. 86

d. Column average for the $7^{\text {th }}$ harmonic freauency domain image of RBSN1. 86

5.6 a. Column average for the $1^{\text {st }}$ harmonic freauency domain image of RBSN2. 87

b. Column average for the $3^{\text {rd }}$ harmonic freauency domain image of RBSN2. 87

c. Column average for the $5^{\text {th }}$ harmonic freauency domain image of RBSN2. 88

d. Column average for the $7^{\text {th }}$ harmonic freauency domain image of RBSN2. 88

A.1 Heat capacity of solid according to the Debye approximation. 98

A.2 Thermal conductivity of nonmetallic material. 100

$\begin{array}{ll}\text { B.1 Model of ta two layer structure. } & 104\end{array}$

B.2 Contour intregral used for the inverse Laplace transform. 109

C.1 Model of a three layer structure. 113 


\begin{abstract}
There is an increasing interest in the characterization of complex structures with thermographic techniques. Typical of these structures are multilayered systems and fiber reinforced composites where structural thermal response is a function of the combined responses of the constituents. In this work, techniques are presented for measuring the early thermal response. For multilayer structures, the response is reduced to the thermal properties of the constituents. For fiber reinforced composites, the short term response enables detection of the fibers.

A measurement system was developed consisting of an infrared detector focused on a single point on the structure. An input heat flux was provided by either an Argon laser or a flash lamp depending on the configuration. To measure the spatial variations in the thermal response, specimens were mounted on a translation stage.

Initial measurements with the system were performed on multilayered structures. An analytical solution was developed for the thermal response of a two or three layer system, given the thermal properties of the layers. This solution was combined with a nonlinear estimation routine to enable the determination of the properties of the constituents from the thermal properties of the structure. This technique was applied to the characterization of a thermally thin layer on a thermally thick layer. It was also applied to the characterization of the center layer of a three layer structure.

Subsequent measurements were performed on a ceramic composite (reaction bonded silicon nitride reinforced with $\mathrm{SiC}$ fibers). Images of the spatial variations in the thermal response were obtained by translation of the composite. To give insights in the significance of the data, a two dimensional analytical solution was developed for the thermal response of a fiber embedded in a matrix material. Predicted responses of the structure were compared with the measured responses.
\end{abstract}


CHARACTERIZATION OF COMPOSITE MATERIALS FROM

TEMPORAL THERMAL RESPONSE 


\section{INTRODUCTION}

In the nineteenth century, Fourier proposed a theory of the propagation of heat in solids. Since, there has been much interest in measuring thermal properties of solids resulting in the development of various methods. Most measurement methods were designed to be implemented on macroscopically homogeneous specimens with a simple geometry. This work extends the possibilities of thermal nondestructive measurement to materials with more complex geometries such as one dimensional flat multilayer or two dimensional composite materials.

For the case of flat plates, techniques were developed to measure thermal properties such as conductivity and diffusivity. Ångström showed that periodic heat flow methods based on the measurement of the phase lag between the excitation and the resulting thermal signal can yield thermal properties [1]. First applied to long thin rods, the method required the recording of two temperature profiles along the sample. Variations based on Ångström's work allowed measurements on samples with a flat geometry [2,3]. More recent developments involving noncontacting detection devices allowed to obtain thermal properties from amplitude and phase frequency characteristics [4]. In the 1980's, photothermal techniques were developed including the optical beam deflection method known as the Mirage effect $[5,6,7]$. A modulated laser thermally excites the specimens. The deflection of a probe laser beam due to the local change of the index of refraction of the 
heated air surrounding the surface provides the temperature measurement. These steady state methods require that the samples reach a dynamic equilibrium which can lead to long measurement times for larger specimens. To remove this difficulty, thermal diffusivity can be determined from analytical solutions which incorporate the transient terms. Often referred to as a standard is the flash method described for the first time by Parker et al [8]. A short duration light pulse is absorbed on one side of a thin slab. Analysis of the resulting temperature history on the other side yields the thermal diffusivity and specific heat of the sample. Other techniques were developed later to remove some limitations, by taking into account, for instance, finite pulse-time and heat loss effects [9]. Advances in thermography have added new possibilities in the field of nondestructive testing. Imaging methods involving flash heating were applied successfully to the detection and characterization in depth and size of defects [10,11]. A Time Resolved Infrared Radiometry (TRIR) technique was recently developed by Murphy et al [12, 13]. It consists of monitoring the surface temperature while a heating pulse is applied. The shape of the temperature-time response provides quantitative information about the material. While the TRIR method was mainly applied to the detection of defects and hidden corrosion on the back surface of aluminum flat plates, a new method described in this work focuses on the nondestructive characterization of thermal properties of multilayered structures. Assuming a planar heat flow, analytical solutions of the temperature that include transient heat conduction were found for two and three layer geometries. To validate the theory, these solutions were compared to the results obtained by numerical methods. Experimental data was taken on samples composed of typical materials used in aerospace applications. The experimental setup used a focused infrared point detector to measure 
the temperature at the surface of the specimens with high temporal and spatial resolution. The material properties in each layer were found by fitting the thermal responses with the analytic solutions.

While multilayered materials are a relatively simple form of composite material, a more complex form consists of fibers embedded in a matrix. The latter is increasingly used in applications that require high strength to weight ratio and resistance to high temperatures. Typical recent applications of advanced composite materials include, for instance, tools for machining and critical parts of heat engines. It was Maxwell in the nineteenth century who started studying the properties of heterogeneous media [14]. His interest was the electrical and magnetic properties. Recent works specifically concerning thermal transfer have led to a better understanding of the relationship between constituents and the relative thermal properties. The main focus, in this case, is to obtain the effective thermal properties of an equivalent homogeneous medium that gives the same averaged thermal response as the composite. Nomura [15] modelled the temperature field, the effective thermal conductivity and specific heat [16] from a study of transient heat conduction that takes into account the geometry of the fibers as well as the entire shape of the composite. Hatta and Taya [17] have used an equivalent inclusion method originally designed for problems in elasticity to solve steady-state conduction problems. They found a solution for the long continuous fiber geometry as a special case of ellipsoidal inclusion. Another important factor in heat conduction in heterogeneous media is the interfacial thermal barrier effect. For the case of spherical inclusions, Benveniste [18] developed a model that allowed thermal contact resistance between constituents in order to obtain an 
expression for the effective thermal conductivity. Hasselman and Johnson [19] also reported that, when the barrier effect was considered, the dispersion size of the dispersed constituent had an influence in the effective thermal properties. They noted that the interfacial barrier effect was only significant if the fibers were nonparallel to the direction of heat flow. They also found that, in that case, the existence of a gap between constituents lowered the thermal conductivity/diffusivity of the composite. Using a flash method to measure thermal properties, Hasselman et al [20] revealed the presence of a thermal barrier effect at the matrix/fiber interface in SiC fiber reinforced Reaction Bonded Silicon Nitride thermally damaged composite samples. Their study revealed that, in the case of absence of contact between the constituents, the heat transfer is due to gaseous conduction at the interface. McCluskey et al, in a study of ceramic-ceramic composite specimens, also measured the thermal properties of the constituents with a flash method [21]. As in previous studies, thermal properties were found to be dependent on the whisker content. The specimens were thermally cycled to induce structural damages, but no significant changes in thermal properties were noted when the cracks developed parallel to the direction of heat flow.

In the present work, the experimental setup used for the nondestructive characterization of multilayered flat plates was modified to image the thermal response of fiber reinforced composite materials. The technique consists in rastering a laser beam, the heat source, at the surface of the specimens. At each point, the temperature is measured as a function of time. A multi-image, composed of the temperature time history at each pixel, is obtained. A model, predicting the temperature response of such composite materials is presented and compared to the experimental data. 
This dissertation consists of 5 parts. Chapter I introduces some basic information such as the equation of heat conduction in a solid body and the classical measurement techniques of thermal properties. Then, in chapter II, models for the new measurement technique predicting the thermal response for one dimensional flat multilayer structures and two dimensional composite materials are developed. Chapter III presents the experimental procedures and describes the specimens studied. Chapter IV shows the experimental results in the form of the typical temperature profiles obtained for multilayer and composite materials. In Chapter V, processed data is presented. Nonlinear least square fitting of the models to the experimental data provides the quantitative information about the material studied. The last part concludes this research.

Also, included at the end are the appendices. Appendix A is a theoretical introduction to the thermal properties of metallic and nonmetallic solids. Appendix B shows the detailed derivations for the approximate solution of the two layer model and appendix C the derivations for the solution of the three layer model. Appendix $D$ is a conference paper describing a diffusivity imaging technique applied to aluminum flat plates, epoxy resin and carbon-carbon composite materials. 


\section{CHAPTER I}

\section{HISTORICAL REVIEW}

The first part of this chapter introduces some background material on heat transfer in solids. The second part is a brief presentation of the existing measurement techniques of thermal properties for simple geometries.

\section{A Definitions and basic equations}

\section{Temperature and heat flux}

Heat can propagate by three different ways:

- radiation, which allows transfer of heat over large distances with or without matter. A body at any temperature greater than absolute zero emits a spectrum of electromagnetic radiation which is dependent on its temperature. This mode only becomes important when large temperature differentials exist between two bodies or a body and its external environment.

- $\underline{\text { conduction, }}$ is the principal transport mode within a solid. Physically, heat can spread through a solid by two different ways. One mechanism involves the vibration of the crystal lattice which dominates the transport in nonmetallic materials. Another is heat transport by free electrons in metallic materials. A theoretical approach of heat 
conduction, as well as some definitions of thermal properties in solids are presented in appendix $A$.

- convection, corresponds to heat transfer from the mass transport within a fluid. A good example are the thermal transfers between a wall and a surrounding fluid like air.

For most applications of interest to nondestructive evaluation, radiation and convection are negligible compared to heat conduction. Because this is also the case for the applications studied in this work, a general theory for heat conduction will be developed in this chapter and applied to specific cases of multilayer and cylindrical geometries.

Any study involving heat conduction in solids requires the knowledge of the temperature field. The temperature above ambient is a scalar function of position and time: $v(\vec{r}, t)$, where $\vec{r}$ is the vector position and the time. When a temperature differential exists in a solid, heat flow takes place. Heat flux is a vector characterized by its magnitude and direction. The magnitude at a given point is defined as the quantity of heat crossing a unit area perpendicular to the direction of heat flow, at the given position, per unit time. The heat flux vector always points towards decreasing temperatures. For a stationary, homogeneous and isotropic solid body, Fourier introduced the basic relationship:

$$
\vec{q}(\vec{r}, t)=-K \nabla v(\vec{r}, t)
$$

where $\vec{q}(\vec{r}, t)$ is the heat flux vector, $\nabla v(\vec{r}, t)$ is the temperature gradient vector and $K$ is 
the thermal conductivity, a scalar quantity that is a property of the material. Thermal conductivity is discussed in greater detail in appendix $\mathrm{A}$.

\section{Differential equation of heat}

An equation for time dependent distribution of heat in a solid can be derived from conservation of energy. Fig. 1.1 shows a stationary, homogeneous, isotropic solid of total volume V, limited on its boundary by the surface $S$.

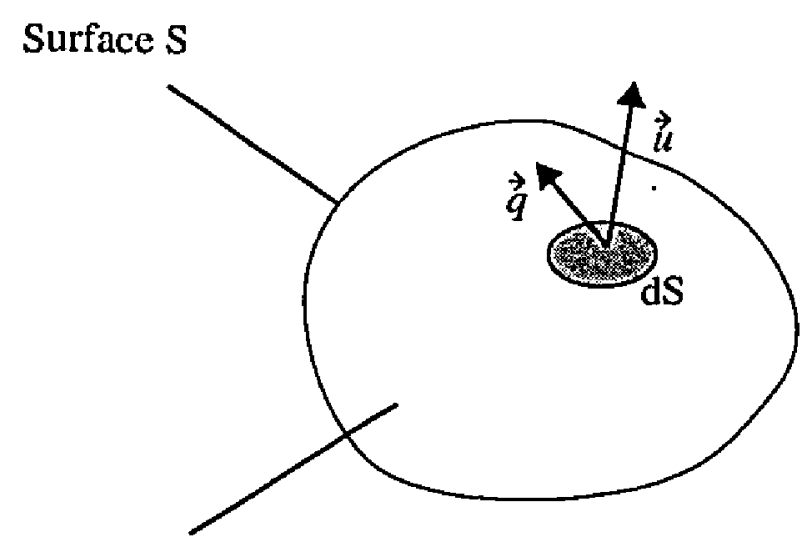

Volume $\mathrm{V}$

Fig. 1.1 Solid of volume V with outer surface $S . \vec{u}$ is a unit outward pointing vector perpendicular to the surface $d S$.

The conservation of energy requires the rate of heat increase within the volume to be equal to the rate of heat generated in the volume plus the heat flow through the surface. This is expressed with the equation:

$$
\frac{d H_{v}}{d t}=F_{s}+G_{v}
$$


where $H_{v}$ is the heat energy in the volume, $F_{s}$ is the rate of heat flow into the volume and $G_{v}$ is the rate of heat generated in the volume.

The rate of heat change in volume $\mathrm{V}$ can be written as:

$$
\frac{d H_{v}}{d t}=\int_{V} \rho C_{p} \frac{\partial v(\vec{r}, t)}{\partial t} d V
$$

where $\rho$ is density and $C_{p}$ the specific heat at constant pressure.

The rate of heat entering volume $V$ through surface $S$ is a surface integral of the flux normal to the surface which can be transformed into a volume integral by using Gauss' theorem:

$$
F_{s}=\int_{S}-(\vec{q}(\vec{r}, t) \cdot \vec{u}) d S=-\int_{V} \operatorname{div}(\vec{q}(\vec{r}, t)) d V
$$

The rate of heat generation in volume $\mathrm{V}$ is:

$$
G_{v}=\int_{V} g(r, t) d v
$$

The conservation of energy gives:

$$
\iint_{V}\left(\rho C_{p} \frac{\partial v(\vec{r}, t)}{\partial t}+\operatorname{div}(\vec{q}(\vec{r}, t))-g(\vec{r}, t)\right) d V=0
$$

By making use of the definition of the heat flux in Eq. (1):

$$
\int_{V}\left(\rho C_{p} \frac{\partial v(\vec{r}, t)}{\partial t}-\nabla \cdot(k \nabla v(\vec{r}, t))-g(\vec{r}, t)\right) d V=0
$$


Eq. (7) is satisfied if the integrand is zero or becomes:

$$
\rho C_{p} \frac{\partial v(\vec{r}, t)}{\partial t}=\nabla \cdot(K \nabla v(\vec{r}, t))+g(\vec{r}, t)
$$

Moreover, if conductivity $K$ is independent from temperature and position:

$$
\frac{1}{\alpha} \frac{\partial v(\vec{r}, t)}{\partial t}=\nabla^{2} v(\vec{r}, t)+\frac{g(\vec{r}, t)}{K}
$$

where $\alpha=\frac{K}{\rho C_{p}}$ is the thermal diffusivity of the material.

Eq. (9) is a general form for the heat equation. However, for most cases of interest, there is no heat generation in the media and Eq. (9) becomes:

$$
\frac{1}{\alpha} \frac{\partial v(\vec{r}, t)}{\partial t}=\nabla^{2} v(\vec{r}, t)
$$

The steady state condition is given by Laplace's equation:

$$
\nabla^{2} v(r, t)=0
$$

\section{B Existing measurement techniques}

The thermal properties that are measured in order to characterize heat transport are thermal conductivity or diffusivity. The heat capacity is also an important thermal property. It is typically measured by calorimetry and will not be presented here because it is not relevant to the present study.

Two classes of techniques have been widely used. While their original implementa- 
tions are simplistic in appearance, they form the basis for all current techniques. Broadly, one can distinguish between the steady state methods to measure thermal conductivity and the variable state methods to determine the thermal diffusivity. The nonsteady state methods were developed later because they are more sophisticated.

\section{Steady state techniques}

Measurements by steady state techniques may involve longitudinal or radial heat flow. The case were heat is generated within the solid is also presented.

\section{a Longitudinal flow}

As shown in Fig. 1.2, heat is supplied at one end of a rod of uniform cross-sectional area $\mathrm{A}$ at a known rate $H$ and is removed at the other end. Thermometers separated by a distance $L$ measure the temperature difference $\Delta v$.

Conductivity $K$ is obtained from the relation:

$$
K=\frac{H L}{A \Delta v}
$$

This method can be used provided that all the heat supplied at one end travels through the specimen to the colder end. For a very poor conductor, radiation losses may be too large to be neglected and the experiment should be conducted with caution. For good conductors, such as metals, heat losses are not a serious problem. 


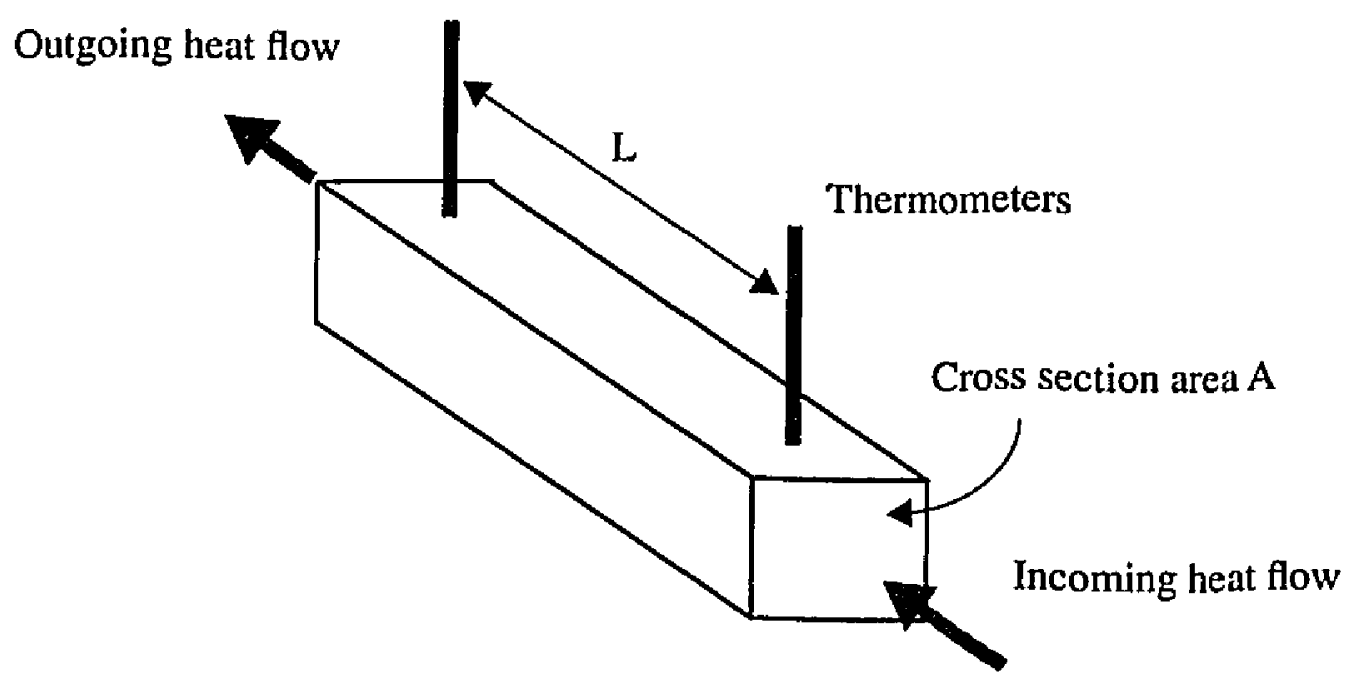

Fig. 1.2 Principle of the steady-state longitudinal heat flow method.

\section{b Radial heat flow}

A heater that is inserted along the axis of a cylinder provides heat uniformly along its length. The thermal conductivity is related to the heat flow per unit length by:

$$
K=\frac{H \ln \left(r_{2} / r_{1}\right)}{2 \pi\left(T_{r_{1}}-T_{r_{2}}\right)}
$$

where $T_{r_{1}}$ and $T_{r_{2}}$ are the temperatures measured at radii $r_{l}$ and $r_{2}$. Practically, the cylinder is composed of a stack of disks to avoid longitudinal flow in the cylinder. In this way, the radial flow is not affected and the longitudinal flow is limited by the interface resistance of the disks. Generating heat internally can be done in many ways, one of them is eddy current heating in a metallic rod with an external coil. 


\section{c Direct heating methods for metals}

A current can generate heat in a metallic sample itself. For a cylinder, the thermal conductivity is related to the difference in temperature between the axis and the surface by:

$$
K=\frac{V_{\varepsilon}^{2} r^{2}}{4\left(T_{a x i s}-T_{r}\right) \rho}
$$

where $V_{\varepsilon}$ is the potential drop along the conductor per unit length, $r$ the radius at the surface and $\rho$ the density.

\section{Variable state techniques}

The variable state methods measure variations in the temperature distribution in a specimen. The rate of change depends on the ratio of the thermal conductivity to the heat capacity per unit volume which is defined as the thermal diffusivity. In general, nonsteady state methods are more difficult to implement than the traditional steady state methods because they require a knowledge of boundary conditions that change during the measurements.

Two types of diffusivity measurements are typically performed in nondestructive evaluation. One involves the application of heat at one location on the sample for a limited time. The diffusivity is determined from the subsequent temperature response at other locations in the specimen. The other type of measurement applies periodic heating to one location and measures the temperature variations at another location.

\section{a Ångström's temperature-wave method}

In this method [1], the temperature at one end of a sample, namely at $x=0$ along the $\mathrm{x}-$ 
axis, is made to vary sinusoidally with frequency $\frac{\omega}{2 \pi}$. The attenuation of the amplitude $v_{O}$ and the time lag of the temperature at the other end is measured. Either of these measurements enables the determination of the thermal diffusivity.

If the input temperature at face $x=0$ of the sample is assumed to be:

$$
v(x, t)=v_{0} \sin (\omega t)
$$

For the no heat losses case, the temperature at a distance $x$ is:

$$
v(x, t)=v_{0} e^{-\sqrt{\frac{\omega}{2 \alpha}} x} \sin \left(\omega t-\sqrt{\frac{\omega}{2 \alpha}} x\right)
$$

where $\alpha$ is the thermal diffusivity of the material.

The decaying exponential factor corresponds to the measurement of the attenuation factor. The term $\sqrt{\frac{\omega}{2 \alpha}} x$ in the sine function corresponds to the measurement of the phase lag.

The diffusivity can be given by :

$$
\alpha=\frac{\omega x^{2}}{2 \ln ^{2}(\Lambda)}=\frac{\omega x^{2}}{2 \psi^{2}}
$$

where $\Lambda$ and $\psi$ are the measured attenuation and phase lag.

Practically, however, both measures are made because, if the experiment is run at room or higher temperatures, the heat losses are not negligible any more. For this case, the 
more accurate measurement of the diffusivity is given by:

$$
\alpha=\frac{\omega x^{2}}{2 \psi \ln (\Lambda)}
$$

An important limitation of the Ångström method is that its practical application involves an excitation source which has a mean temperature that is different from the initial temperature of the specimen. The periodic variation in temperature is therefore superimposed on a much longer variation as the mean temperature of the specimen approaches the mean temperature of the source. Two techniques can separate these two variations. One is to allow the specimen to come to the mean temperature of the source before performing the measurement. The other consists of performing a band pass filter on the temperature measurement to isolate the periodic response. Both of these measurements may require a significant time if the sample is large.

\section{b Transitory heating methods}

Typical of this type of method is Parker's [8] which involves a short pulse of radiant heat with a laser or a flash tube and monitoring of the variations in temperature at the opposite face of the sample. For plate geometries, this allows the determination of the diffusivity with a simple computation. The measurement relative to periodic technique is short in duration enabling the heat losses by convection to be neglected. Depending on the geometry of the sample, a relationship between the time to attain a desired temperature and the diffusivity is obtained:

$$
\alpha=1.38 \frac{L^{2}}{\pi^{2} t_{1 / 2}}
$$


where $L$ is the flat plate thickness and $t_{I / 2}$ the time required for the back surface to reach half the maximum temperature rise. 


\section{CHAPTER II}

\section{THEORETICAL MODELS FOR NEW MEASUREMENT TECHNIQUE}

The first chapter introduced some background material on heat transfer in solids followed by a brief presentation of the existing measurement techniques of thermal properties. With such techniques, difficulties arise for large samples. The steady state methods require that the samples reach a dynamic equilibrium which can lead to long measurement times. Moreover, the existing transitory heating methods only apply to macroscopically homogeneous specimens with a simple geometry. Improved characterization of new materials and structures requires new methods. To enable this, it is necessary to develop models to predict the temperature response of complex structures. This chapter presents models of the transient temperature response for flat multilayered structures and fiber reinforced composites.

\section{A Multilayer structures}

Multilayer structures are broadly used in industrial applications and include, for instance, all riveted structures. For such geometries, Laplace transforms are used to solve the heat equation. A frequency domain solution can be found easily for any number of layers, but inversion of the solutions by the standard complex integral method is not trivial. An expansion technique is used to obtain the temperatures in the case of two and three 
layers. Simulations are compared to results obtained from a numerical inversion technique.

\section{Frequency domain solutions}

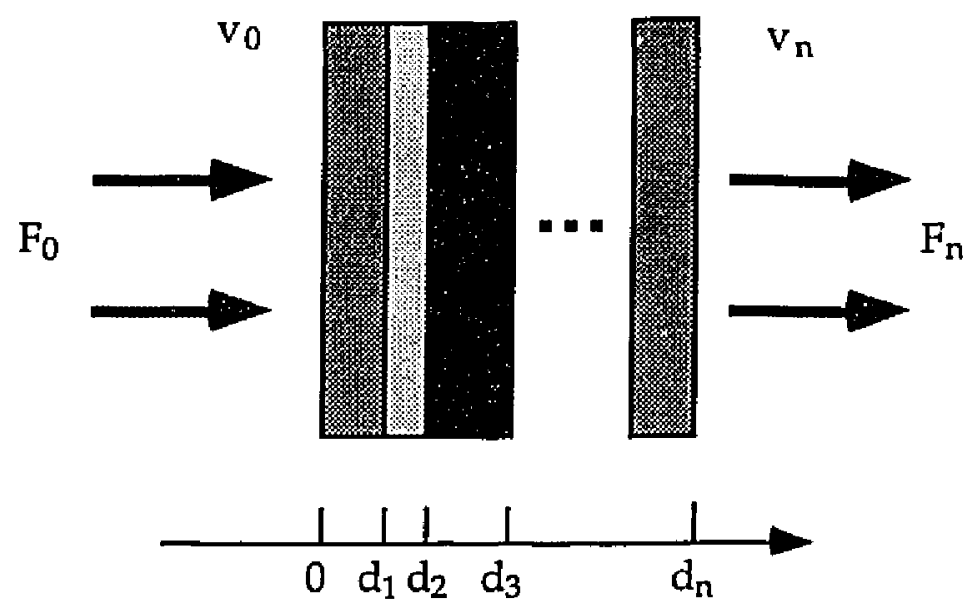

Fig. 2.1 Model for a multilayer structure.

The multilayered structure is represented by the one dimensional model shown in Fig 2.1. Layer $\mathrm{r}$ has a thermal diffusivity $\alpha_{r}$ and conductivity $K_{r}$ and is in perfect thermal contact with adjacent layers. The heat fluxes at the front and back surfaces are given by $F_{0}$ and $F_{n}$ respectively. Convection losses have been neglected and the resulting temperatures at the front and back surfaces are $v_{0}$ and $v_{n}$.

The general relationship between the Laplace transforms of the flux and the temperature on each side of a multilayer slab [22] is given by Eq. (1):

$$
\left[\begin{array}{l}
\bar{v}_{n} \\
\overline{F_{n}}
\end{array}\right]=T_{n} \cdot \ldots \cdot T_{r} \cdot \ldots \cdot T_{2} \cdot T_{1}\left[\begin{array}{c}
\overline{v_{0}} \\
\overline{F_{0}}
\end{array}\right]
$$


where the transfer matrix for the $\mathrm{r}^{\text {th }}$ layer is given by:

$$
T_{r}=\left[\begin{array}{cc}
\cosh \left(l_{r} q_{r}\right) & -\frac{\sinh \left(l_{r} q_{r}\right)}{K_{r} q_{r}} \\
-K_{r} q_{r} \sinh \left(l_{r} q_{r}\right) & \cosh \left(l_{r} q_{r}\right)
\end{array}\right] \quad \text { with } \quad q_{r}=\sqrt{\frac{p}{\alpha_{r}}}
$$

where $p$ is the Laplace variable, and $l_{r}=d_{r}-d_{r-1}$ is the thickness of the layer $\mathrm{r}$.

For the case of interest, the fiux is only applied to a single surface and the heat losses at the opposite surface are neglected, reducing Eq. (1) to:

$$
\left[\begin{array}{l}
\overline{v_{n}} \\
0
\end{array}\right]=\left[\begin{array}{ll}
A & B \\
C & D
\end{array}\right]\left[\begin{array}{l}
\overline{v_{0}} \\
\overline{F_{0}}
\end{array}\right]
$$

where $A, B, C$ and $D$ are obtained by multiplying the 2 by 2 transfer matrices. They depend on the thicknesses and the thermal properties of the $\mathrm{n}$ layers. If at least one layer has non-zero heat capacitance, $C$ is non-zero. Then, the Laplace transforms of the temperatures at the front and back surfaces are respectively given by:

$$
\overline{v_{0}}=\left(-\frac{D}{C}\right) \overline{F_{0}}
$$

and

$$
\overline{v_{n}}=\left(B-\frac{A D}{C}\right) \overline{F_{0}}
$$

There is no particular difficulty in the computation of $A, B, C$ and $D$. However, even for a two layer system, inversion by complex integration is non trivial. This is illustrated by Eq. (6) which is the Laplace transform of the front surface temperature for a two layer 
structure with constant heat flux $F_{0}$ starting at $t=0$.

$$
\frac{\cosh \left(l_{1} q_{1}\right) \cosh \left(l_{2} q_{2}\right)+\frac{K_{2} q_{2} \sinh \left(l_{1} q_{1}\right) \sinh \left(l_{2} q_{2}\right)}{K_{1} q_{1}}}{\left.\left.K_{1} q_{1} \cosh \left(l_{2} q_{2}\right) \sinh \left(l_{1} q_{1}\right)\right)-K_{2} q_{2} \cosh \left(l_{1} q_{1}\right) \sinh \left(l_{2} q_{2}\right)\right)} F_{0}
$$

Inversion is performed by numerical routine or by expansion of the Laplace transforms to series with simpler terms that can be analytically inverted.

\section{Inversion to time domain by the expansion method}

\section{a Two layer model}

A case of practical importance is a thermally thin layer backed by a second thermally thick layer. It includes coated or painted structures. For this case with a constant incoming heat flux $F_{O}$ starting at time $t=0$, the long time solution is found by expanding $\bar{v}_{O}$ about $q_{/}$equal to zero $(t \rightarrow \infty)$. The first order of the expansion approximates the first layer as a contact resistance (i.e. without specific heat). The transfer matrix for the first layer has the form:

$$
T_{1}=\left[\begin{array}{cc}
1+O\left(q_{1}^{2}\right) & -\left(\frac{l_{1}}{K_{1}}\right)+O\left(q_{1}^{2}\right) \\
O\left(q_{1}^{2}\right) & 1+O\left(q_{1}^{2}\right)
\end{array}\right]
$$

The first order approximation for $\overline{v_{0}}$ is:

$$
\overline{v_{0}}=F_{0}\left(\frac{l_{1}}{K_{1} p}+\frac{\operatorname{coth}\left(l_{2} q_{2}\right)}{K_{2} q_{2} p}\right)+O\left(q_{1}^{2}\right)
$$


Inverting the Laplace transform $\bar{v}_{O}$ in Eq. (8) gives a first order approximation for $v_{0}$ :

$$
v_{0}=F_{0}\left(\frac{l_{1}}{K_{1}}+\frac{2 \sqrt{\alpha_{2} t}}{K_{2}} \sum_{n=0}^{\infty}\left(\operatorname{ierfc}\left(\frac{n l_{2}}{\sqrt{\alpha_{2} t}}\right)+i e r f c\left(\frac{(n+1) l_{2}}{\sqrt{\alpha_{2} t}}\right)\right)\right)
$$

where ierfc is the integral complimentary Error function. At $t=0$, the first term of this solution results in an instantaneous jump proportional to the heat flux. This is a result of the approximation of the first layer having no heat capacitance. The second term is equivalent to the thermal response of the second layer for the case of no first layer. The initial discontinuity in the solution makes comparison with data difficult because the model cannot represent it accurately.

In the third order approximation, the first layer is a thermally thin layer and $\bar{v}_{0}$ is still simple to invert. The transfer matrix for the first layer becomes:

$$
T_{1}=\left[\begin{array}{cc}
1+\frac{l_{1}^{2} q_{1}^{2}}{2}+O\left(q_{1}^{4}\right) & -\frac{l_{1}}{K_{1}}-\frac{l_{1}^{3} q_{1}^{2}}{6 K}+O\left(q_{1}^{4}\right) \\
-K_{1} l_{1} q_{1}^{2}+O\left(q_{1}^{4}\right) & 1+\frac{l_{1}^{2} q_{1}^{2}}{2}+O\left(q_{1}^{4}\right)
\end{array}\right]
$$

The third order approximation for $\bar{v}_{0}$ is:

$$
\begin{aligned}
& \bar{v}_{0}=F_{0}\left(\frac{l_{1}}{K_{1} p}+\frac{\operatorname{coth}\left(l_{2} q_{2}\right)}{K_{2} q_{2} p}\right)+\frac{l_{1} q_{1}^{2} \operatorname{csch}^{2}\left(l_{2} q_{2}\right)}{6 K_{1} K_{2}^{2} q_{2}^{2} p}\left(-3 K_{1}^{2}\left(1+\cosh \left(2 l_{2} q_{2}\right)\right)\right. \\
& \left.+K_{2}^{2} l_{1}^{2} q_{2}^{2}\left(1-\cosh \left(2 l_{2} q_{2}\right)\right)-3 K_{1} K_{2} l_{1} q_{2} \sinh \left(2 l_{2} q_{2}\right)\right)+O\left(q_{1}^{4}\right)
\end{aligned}
$$

where the first term is the first order approximation. The remaining terms can be inverted one by one. Eq. (12) shows the solution where Erf is the Error function. The first term of Eq. (12) is equivalent to Eq. (9), the second line is the second term of the expansion. 


$$
\begin{aligned}
& v_{0}=F_{0}\left(\frac{l_{1}}{K_{1}}+\frac{2 \sqrt{\alpha_{2} t}}{K_{2}}\left(1+2 \sum_{n=1}^{\infty} \operatorname{ierfc}\left(\frac{n l_{2}}{\sqrt{\alpha_{2} t}}\right)\right)\right. \\
& -\frac{\alpha_{2} l_{1}^{2}}{l_{2} K_{2} \alpha_{1}}\left(1+2 \sum_{n=1}^{\infty} e^{\left.-\left(\frac{n^{2} \pi^{2} \alpha_{2} t}{l_{2}^{2}}\right)\right)}-\frac{K_{1} l_{1} \alpha_{2}}{K_{2}^{2} \alpha_{1}} \sum_{n=1}^{\infty} 4 n\left(1-\operatorname{Erf}\left(\frac{n 1_{2}}{\sqrt{\alpha_{2} t}}\right)\right)\right)
\end{aligned}
$$

\section{b Three layer model}

For the case of a three layer structure with flash heating, a through-the-thickness measurement simplifies the characterization of the inner layer. The early time expansion of $\bar{v}_{3}$ is given by expanding about $e^{-l q}$ equal to zero $(p \rightarrow \infty)$. This corresponds to an expansion about $t=0$. Assuming the first and last layers are identical, the temperature $\bar{v}_{3}$ is given by:

$$
\begin{gathered}
\overline{v_{3}}=\left(\frac{4 K_{2}}{\alpha_{2} \sqrt{p}}\right) /\left(\left(\frac{K_{1}}{\sqrt{\alpha_{1}}}+\frac{K_{2}}{\sqrt{\alpha_{2}}}\right)^{2} \sinh \left(\sqrt{p}\left(\frac{2 l_{1}}{\sqrt{\alpha_{1}}}+\frac{l_{2}}{\sqrt{\alpha_{2}}}\right)\right)\right. \\
\left.-\left(\frac{K_{1}}{\sqrt{\alpha_{1}}}-\frac{K_{2}}{\sqrt{\alpha_{2}}}\right)^{2} \sinh \left(\sqrt{p}\left(\frac{2 l_{1}}{\sqrt{\alpha_{1}}}-\frac{l_{2}}{\sqrt{\alpha_{2}}}\right)\right)+2\left(\frac{K_{2}^{2}}{\alpha_{2}}-\frac{K_{1}^{2}}{\alpha_{1}}\right) \sinh \left(\frac{l_{2} \sqrt{p}}{\sqrt{\alpha_{2}}}\right)\right)
\end{gathered}
$$

It is possible to evaluate the coefficients of the series expansion of $\bar{v}_{3}$. Inverting term by term gives a solution for $v_{3}$ shown in Eq. (14) and (15):

$$
v_{3}=\frac{8 F_{0} K_{2}}{\sqrt{\pi \alpha_{2} t}}\left(\sum_{n=0}^{\infty} \sum_{n=0}^{\infty} \psi_{e} \frac{\left((2 n+1) \frac{l_{2}}{\sqrt{\alpha_{2}}}+(m+1) \frac{l_{1}}{\sqrt{\alpha_{1}}}\right)^{2}}{4 t}\right)
$$

where 


$$
\begin{aligned}
& \psi=\sum_{i=0}^{m}\left(\left(\sum_{j=0}^{m-i}\left(\begin{array}{l}
n \\
j
\end{array}\right)\left(\begin{array}{c}
j \\
m-j-i
\end{array}\right)\left(\frac{K_{1}}{\sqrt{\alpha_{1}}}+\frac{K_{2}}{\sqrt{\alpha_{2}}}\right)^{2(m-i-j)}\left(\frac{K_{1}}{\sqrt{\alpha_{1}}}-\frac{K_{2}}{\sqrt{\alpha_{2}}}\right)^{2(n-j)}\left(\left(\frac{K_{1}^{2}}{\alpha_{1}}-\frac{K_{2}^{2}}{\alpha_{2}}\right)\right)^{i+2 j-m}\right)\right. \\
& \left.\left(\sum_{s=0}^{i}(-1-n)\left(\begin{array}{c}
s \\
s-s
\end{array}\right)\left(\frac{K_{1}}{\sqrt{\alpha_{1}}}+\frac{K_{2}}{\sqrt{\alpha_{2}}}\right)^{2(-1-n-s)}\left(\frac{K_{1}}{\sqrt{\alpha_{1}}}-\frac{K_{2}}{\sqrt{\alpha_{2}}}\right)^{2(i-s)}\left(2\left(\frac{K_{1}^{2}}{\alpha_{1}}-\frac{K_{2}^{2}}{\alpha_{2}}\right)\right)^{-i+2 s}\right)\right)
\end{aligned}
$$

The analytical solution proposed in Eq.(14) can be interpreted in terms of thermal path lengths. As Fig. 2.2 shows, each term in the doubly infinite series can be assigned to a thermal path in the three layer structure. $n$ determines the number of reflections in the inner layer, $m$ in the outer ones. There are multiple possibilities for $m>0$.

Unlike the propagation of sound, the diffusion of heat in solids is strongly attenuated. In practice, very few reflections are to be taken into account to accurately represent the temperature at the surface.

\begin{tabular}{|c|c|}
\hline $\mathbf{n}$ & $\mathrm{m}$ \\
\hline 0 & 0 \\
0 & 1 \\
0 & 2 \\
1 & 0 \\
2 & 0 \\
\hline
\end{tabular}

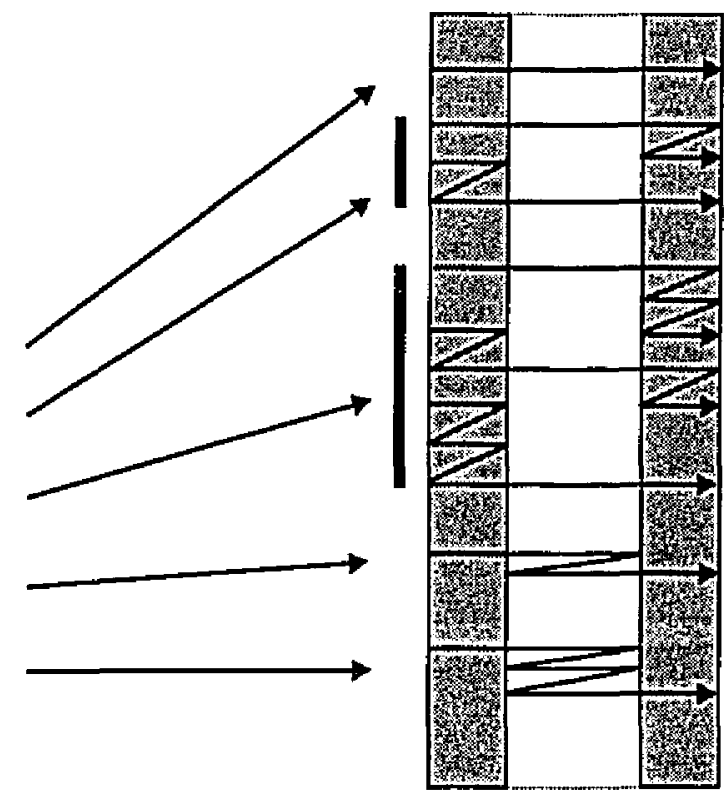

Fig. 2.2 Interpretation of the analytical solution for $v_{3} . m$ and $n$ are the summation indices in Eq. (14) and (15). 


\section{Simulations}

The model for two layer structures has an intrinsic limitation. The technique involves an expansion of the Laplace transforms of the temperatures. For simplicity, only the first two terms are inverted. As a consequence, the surface layer is assumed to be thermally thin. When the excitation frequency is too high for a significant amount of heat to reach the second layer, the model does not apply anymore.

Another limitation comes from the mathematical nature of the solutions. For both two and three layer structures, the solution has the form of an infinite series. Practically, a choice concerning the number of terms to be evaluated is necessary.

The following part discusses the accuracy of the expansion method from examples of predicted temperature profiles. The results obtained with the method were compared to those obtained with a numerical inversion technique for different sets of parameters.

\section{a Numerical inversion}

A numerical inversion method is used in this work as a validation for the expansion method. For the case of Laplace transforms applied to the diffusion equation, instability problems arise when discrete data are inverted. This is due to the smoothing action of the forward transform. As time moves forward, high frequency components of the solutions are more strongly attenuated. This is why an initial temperature profile becomes progressively smoother with time [26]. From a computational point of view, this is not a problem for the forward transform but the inversion process uses all frequencies. Typically, data noise can be mistaken for high frequency information. As a consequence, numerical inversion of Laplace transform is a ill-posed problem and care must be taken in using such 
methods. It is expected that the error increases with time. However, this is not a serious concern because typical transient thermal responses are short.

The Laplace transforms $\bar{v}_{0}$ and $\bar{v}_{n}$ shown in Eq. (4) and (5) are evaluated and provided to a numerical inversion routine based on Stehfest method [24]. Given a Laplace transform $P(s)$, the inversion produces an approximate value $F_{a}$ of the inverse $F(t)$ at time $T$ :

$$
F_{a}=\frac{\ln 2}{T} \sum_{i=1}^{N} V_{i} P\left(\frac{\ln 2}{T} i\right)
$$

where $V_{i}$ depends on $N$ only.

\section{b Comparison}

Fig. 2.3 shows the predicted back surface temperature profiles for a three layer structure with unit flash heating at $t=0$ at the front. Since, in this case, both methods have errors increasing with time, a good agreement is expected for $t$ close to zero. To obtain an accurate result for longer times, more terms are needed in the expansions. The temperature profiles in Fig. 2.3 (a) are practically identical. However, they diverge in Fig. 2.3 (b) after 3 seconds. This is due to the increased thermal properties mismatch between the outer and inner layers. The increased outer layer's thermal diffusivity and lower thermal conductivity of the inner layer in Fig. 2.3 (b) are responsible for the faster time of the thermal response and its larger amplitude. Table 2.1 provides the thermal properties used in the simulations. Fig. 2.4 shows the predicted front side temperature profiles for a two layer structure with constant flux heating starting at $t=0$. The properties used for both Fig. 2.4 (a) and (b) are identical. They are given in Table 2.2. In this case, because the expansion is performed about $\mathrm{e}^{-\mathrm{lq}}$ equal to zero, a good agreement is expected for long times. 
An increasing number of terms are needed in the expansions for accurate results for $t$ close to zero. Fig. 2.4 (b) shows the first $0.1 \mathrm{~ms}$ of the simulations in Fig. 2.4 (a). The expansion model diverges from the numerical inversion for $t<0.03 \mathrm{~ms}$. For longer times, the expansion and the numerical models give identical results.

Fig. 2.3 and 2.4 display typical behaviors of numerical and expansion methods. For the three layer model, the accurate results are expected for $t$ close to zero. For the two layer model, the temperature is best estimated for long times. For least square fitting using both models, care must be taken to include a sufficient numbers of terms to accurately represent the temperature profiles for the estimated parameters. Including too many terms does not bring more information and slows down the least square fitting algorithm. 


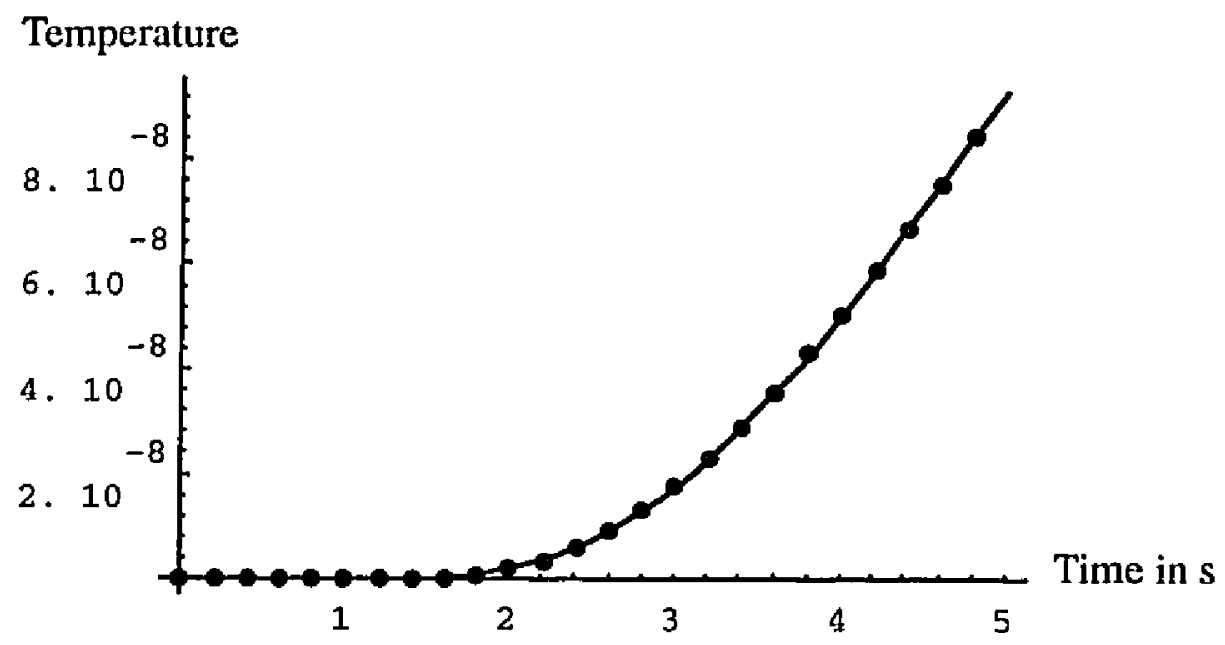

(a)

Temperature

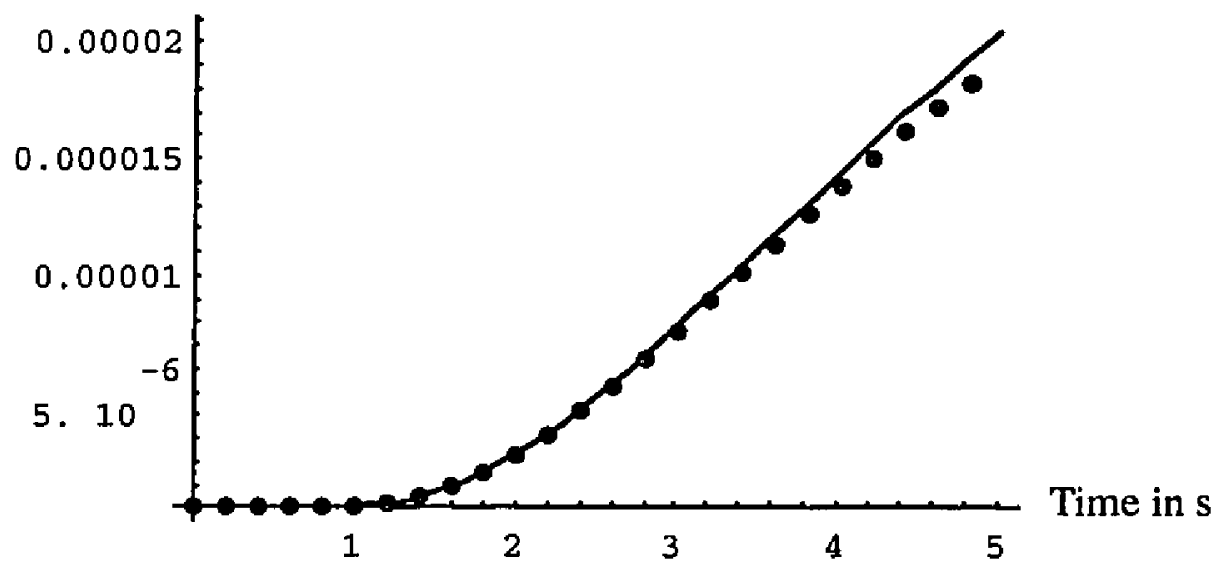

(b)

Fig. 2.3 (a,b). Simulated time temperature profiles for two sets of parameters for three layer structure with unit flash heating at $t=0$. The temperature profile in dots were obtained by the expansion method, the ones in continuous line by numerical inversion. Temperature differences are in Kelvin per unit flux. 


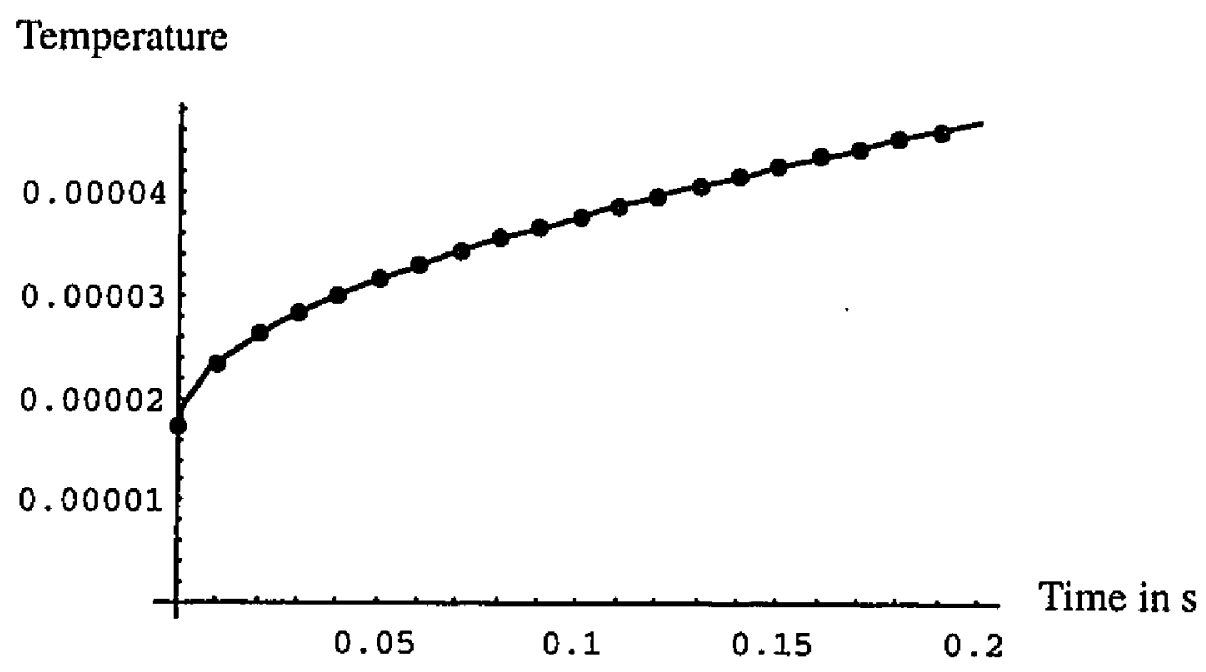

(a)

\section{Temperature}

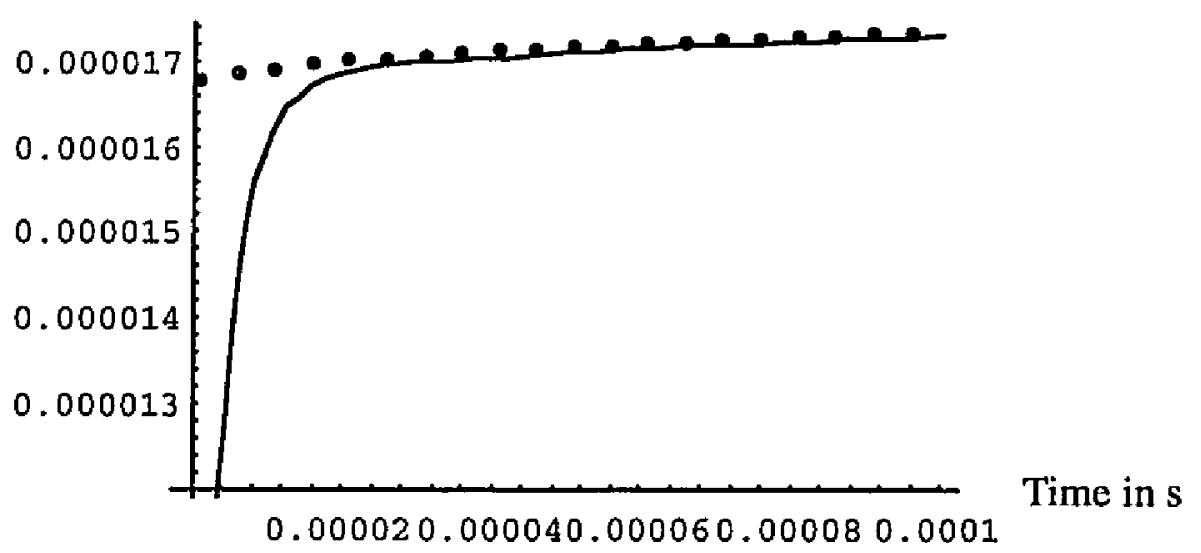

(b)

Fig. 2.4 (a,b). Simulated time temperature profiles for one set of parameters for a two layer structure with unit constant heating starting at $t=0$. The temperature profiles in dots were obtained by the expansion method, the ones in continuous line by numerical inversion. Temperature differences are in Kelvin per unit flux. 


\begin{tabular}{|l|r|r|r|r|}
\hline & \multicolumn{2}{|c|}{ Model (a) } & \multicolumn{2}{c|}{ Model (b) } \\
\hline & outer layers & inner layer & outer layers & inner layer \\
\hline \hline Layer thickness in $\mathrm{mm}$ & 1.016 & 0.5 & 1.016 & 0.5 \\
\hline $\begin{array}{l}\text { Thermal diffusivity } \\
\text { in } \mathrm{m}^{2} / \mathrm{s}\end{array}$ & $0.610^{-6}$ & $0.0110^{-6}$ & $8610^{-6}$ & $0.0110^{-6}$ \\
\hline $\begin{array}{l}\text { Thermal conductivity } \\
\text { in W/m K }\end{array}$ & 230 & 30 & 230 & 0.3 \\
\hline
\end{tabular}

Table 2.1 Thermal properties for simulations (a) and (b) in Fig. 2.3.

\begin{tabular}{|l|r|r|}
\hline & first layer & \multicolumn{1}{c|}{ second layer } \\
\hline \hline Layer thickness in $\mathrm{m}$ & $510^{-6}$ & $510^{-3}$ \\
\hline Thermal diffusivity in $\mathrm{m}^{2} / \mathrm{s}$ & $310^{-6}$ & $5010^{-6}$ \\
\hline Thermal conductivity in $\mathrm{W} / \mathrm{m} \mathrm{K}$ & 0.3 & 120 \\
\hline
\end{tabular}

Table 2.2 Thermal properties for simulation in Fig. 2.4

\section{B Solutions for cylindrical geometry}

There has been an increasing interest in the thermal characterization of fiber reinforced composite materials. This section presents a two dimensional solution for the temperature response of a fiber embedded in a matrix to a periodic point heat source. The response is found by expanding the thermal excitation function in terms of cylindrical functions. For the case of multiple fibers, a closed form solution is not available. An approximate solution can be obtained by superposition of the individual solutions. 
In the last part of this section, surface plots of the temperature field in the matrix with an embedded fiber and temperature profiles for different parameters are shown and discussed

\section{Thermal response of single fiber embedded in matrix}

Fig. 2.5 shows the geometry, in two dimensions, of the studied system. It is assumed that material 1 (matrix) and 2 (fiber) are homogeneous, isotropic and in perfect thermal contact with each other. However, the material properties of the matrix and fiber are allowed to be arbitrarily different from each other, and the size and distance from the heat source of the fiber are not restricted.

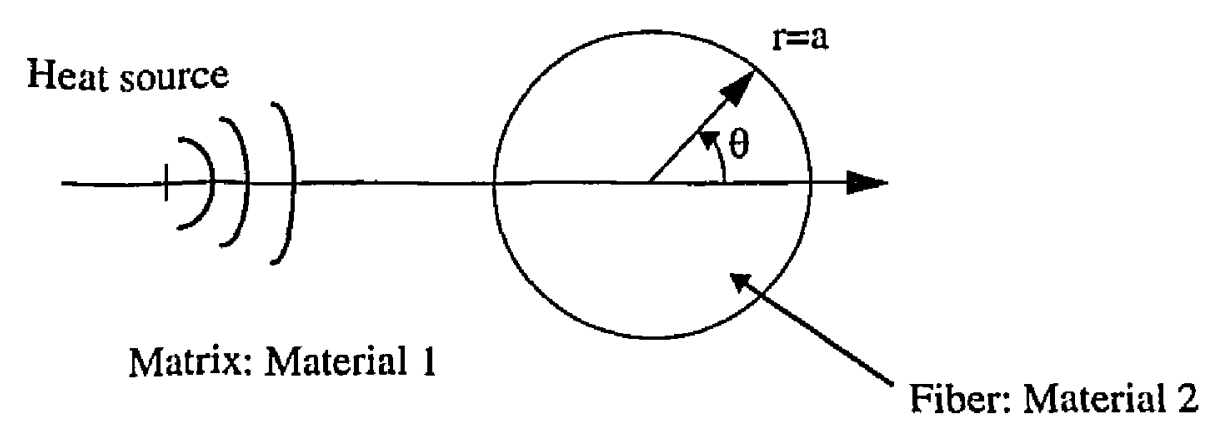

Fig. 2.5 Geometry for 2 dimensional one fiber composite system and periodic point heat source.

The thermal response for a source with a time dependence given by $e^{i \omega t}$ is $v(x) e^{i \omega t}$, where $v(x)$ is the solution to:

$$
\left(\nabla^{2}-\frac{i \omega}{\alpha}\right) v(x)=0
$$


where $v(x)$ is spatial temperature dependence. Let $k=\sqrt{\frac{\omega}{i \alpha}}$, Eq. (17) becomes Helmholtz's equation:

$$
\nabla^{2} v(x)+k^{2} v(x)=0
$$

In a cylindrical coordinate system, Eq. (18) has general solution of the form:

$$
v=\sum_{n=0}^{\infty} \varepsilon_{n} A_{n}\left(\begin{array}{l}
H_{n}^{(1)}(k r) \\
H_{n}^{(2)}(k r)
\end{array}\right)\left(\begin{array}{c}
\cos n \theta \\
\sin n \theta
\end{array}\right)
$$

where $H_{n}^{(1)}(k r)$ and $H_{n}^{(2)}(k r)$ are the Hankel functions of the first and second kind.

The brackets indicate that the general solution is a linear combination of the terms shown. $\varepsilon_{n}$ has value $l$ for $n=0$ and 2 otherwise.

The temperature field in the matrix can be written down as the sum of the temperature field due to the heat source and of the temperature response due to the fiber. Solution in the matrix is:

$$
v_{1}=v_{\text {incoming }}+\sum_{n=0}^{\infty} \varepsilon_{n} B_{n} H_{n}^{(2)}\left(k_{1} r\right)\left(\begin{array}{c}
\cos n \theta \\
\sin n \theta
\end{array}\right)
$$

where $k_{1}=\sqrt{\frac{\omega}{i \alpha_{1}}}$ and the Hankel function of the second kind is chosen so that the temperature field tends to zero when $r$ tends to infinity. The disturbance caused by the fiber must die out at large distances. 
For the two dimensional case, the temperature distribution due to a point source [23] is:

$$
v_{\text {incoming }}=\frac{K_{0}\left(i k_{1}\left|\vec{r}-\vec{r}_{0}\right|\right)}{2 \pi \alpha_{1}}=\frac{i}{4 \alpha_{1}} H_{0}^{(1)}\left(-k_{1}\left|\vec{r}-\vec{r}_{0}\right|\right)
$$

where $\vec{r}_{0}$ is the position of the source. Fig. 2.6 shows the geometry for a point source located at $S_{0}$.

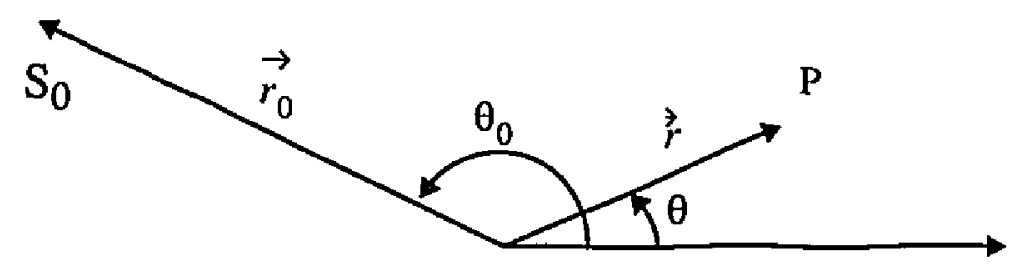

$$
R=\left|\overrightarrow{r_{0}}-\vec{r}\right|
$$

Fig. 2.6 Point source at $S_{0}$ and expansion at point $P$.

$v_{\text {incoming }}$ can be expanded in terms of functions centered at the origin [25]. For

$\left|\overrightarrow{r_{0}}\right|>|\vec{r}|$, the expansion takes the form:

$$
H_{0}(k R)=\sum_{n=0}^{\infty} \varepsilon_{n} H_{n}\left(k r_{0}\right) J_{n}(k r) \cos \left(n\left(\theta-\theta_{0}\right)\right)
$$

where $\left(r_{0}, \theta_{0}\right)$ are the polar coordinates for $\vec{r}_{0}$. In the present case, the heat source is located at $\theta_{0}=\pi$ :

$$
v_{\text {incoming }}=\frac{i}{4 \alpha_{1}} \sum_{n=0}^{\infty} \varepsilon_{n} H_{n}^{(1)}\left(-k_{1} r_{0}\right) J_{n}\left(-k_{1} r\right) \cos (n(\theta-\pi))
$$


Since $H_{n}^{(1)}\left(k_{1} r_{0}\right)$ is known, $v_{\text {incoming }}$ can be more conveniently rewritten as:

$$
v_{\text {incoming }}=\sum_{n=0}^{\infty} \varepsilon_{n} D_{n} J_{n}\left(-k_{1} r\right) \cos n \theta
$$

where $D_{n}=\frac{(-1)^{n} i H_{n}^{(1)}\left(-k_{1} r_{0}\right)}{4 \alpha_{1}}$

Solution within the fiber is, since the temperature at the center of the fiber is finite:

$$
v_{2}=\sum_{n=0}^{\infty} \varepsilon_{n} C_{n} J_{n}\left(-k_{2} r\right)\left(\begin{array}{c}
\cos n \theta \\
\sin n \theta
\end{array}\right)
$$

where $k_{2}=\sqrt{\frac{\omega}{i \alpha_{2}}}$.

At the interface between the fiber and the matrix, there is continuity of temperature and flux, which is expressed by:

$$
\left.v_{1}\right|_{r=a}=\left.v_{2}\right|_{r=a}
$$

and

$$
\left.K_{1} \frac{\partial v_{1}}{\partial r}\right|_{r=a}=\left.K_{2} \frac{\partial v_{2}}{\partial r}\right|_{r=a}
$$

Initially, the two equations that correspond to the boundary conditions appear complicated since they involve infinite summations over index $n$. However, due to the orthogonality of the sine and cosine functions, the expansions must be equal term by term. The condition on temperature gives, for any $n$ : 


$$
D_{n} J_{n}\left(-k_{1} a\right)+B_{n} H_{n}^{(2)}\left(k_{1} a\right)=C_{n} J_{n}^{\left(-k_{2} a\right)}
$$

The condition on flux gives:

$$
-k_{1} K_{1} D_{n} J_{n}^{\prime}\left(-k_{1} a\right)+k_{1} K_{1} B_{n} H_{n}^{(2)}{ }^{\prime}\left(k_{1} a\right)=-k_{2} K_{2} C_{n} J_{n}^{\prime}\left(-k_{2} a\right)
$$

where the prime defines the derivative with respect to the variable. For instance:

$$
J_{n}{ }^{\prime}\left(k_{1} a\right)=\left.\frac{\partial}{\partial\left(k_{1} r\right)} J_{n}\left(k_{1} r\right)\right|_{r=a}
$$

It is now possible to write the two boundary condition equations as a matrix system:

$$
\left[\begin{array}{cc}
H_{n}^{(2)}\left(k_{1} a\right) & -J_{n}\left(-k_{2} a\right) \\
k_{1} K_{1} H_{n}^{(2)}\left(k_{1} a\right) & k_{2} K_{2} J_{n}^{\prime}\left(-k_{2} a\right)
\end{array}\right]\left[\begin{array}{l}
B_{n} \\
C_{n}
\end{array}\right]=D_{n}\left[\begin{array}{c}
-J_{n}\left(-k_{1} a\right) \\
k_{1} K_{1} J_{n}^{\prime}\left(-k_{1} a\right)
\end{array}\right]
$$

The solution to this system is:

$$
B_{n}=D_{n} \frac{-k_{2} K_{2} J_{n}\left(-k_{1} a\right) J_{n}{ }^{\prime}\left(-k_{2} a\right)+k_{1} K_{1} J_{n}{ }^{\prime}\left(-k_{1} a\right) J_{n}\left(-k_{2} a\right)}{k_{1} K_{1} J_{n}\left(-k_{2} a\right) H_{n}^{(2){ }^{\prime}}\left(k_{1} a\right)+k_{2} K_{2} H_{n}^{(2)}\left(k_{1} a\right) J_{n}{ }^{\prime}\left(-k_{2} a\right)}
$$

and

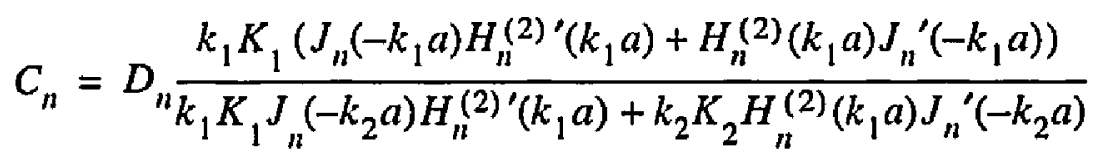

The temperature responses are obtained by replacing $B_{n}$ and $C_{n}$ in the expressions for $v_{l}$ and $v_{2}$. In the limiting case where the properties for matrix and fiber are the same, $B_{n}$ becomes zero for any $n$ and Eq. (33) becomes $C_{n}=D_{n}$. The scattered field due to the properties mismatch vanishes. In the matrix, the temperature field is due to the heat 
source only. In the fiber, the temperature is given by Eq. (25) where $C_{n}$ is replaced by $D_{n}$ :

$$
v_{2}=\sum_{n=0}^{\infty} \varepsilon_{n} D_{n} J_{n}\left(-k_{2} r\right) \cos n \theta
$$

and, since $k_{l}=k_{2}$, Eq. (34) is the expression for $v_{\text {incoming. }}$.

\section{Theoretical response for multiple fibers embedded in a matrix}

The thermal response for multiple fibers is given by the superposition of the thermal response of each of the fibers, when the separation of the fibers is large relative to the inverse of $k_{l}$. Within this limit, the interaction between the fibers can be neglected. This condition is achieved by increasing the frequency of the heat source until only one or two fibers are in the region of significant thermal excitation.

\section{Simulations}

In general, for complex geometries such as the one representing fiber reinforced composites, no closed form solutions for the temperature field are available. Instead, the expressions giving the temperature are an infinite series. Fortunately, this is not too limiting an approximation. Typically, the terms in the series quickly decay and it is not necessary to consider a large number to obtain convergence. Potential problems arise from the assumptions that were made in the formulation of the model. For instance, fiber and matrix are assumed to be in perfect thermal contact with each other (i.e: continuity of temperature and flux across the boundary). If it is a reasonable approximation in the case of 
the original specimens where fibers are in intimate contact with the surrounding matrix, it is not for the thermally damaged ones. For Reaction Bonded Silicon Nitride composites reinforced with $\mathrm{SiC}$ fibers, other studies $[19,20]$ have revealed the existence of a carbon depleted region at the interface between constituents of heat damaged specimens. Effectively, disbonding occurs between the components. Predicted data must then be understood in terms of effective properties, the equivalent properties for a degraded fiber in perfect thermal contact with the matrix. This is mainly an interpretation problem.

Another approximation is made when the propagation of heat is assumed to be 2dimensional whereas it is really 3 -dimensional. But taking into account the planar heat flow in the direction of the fibers leads to a much more complicated problem. The heat source term must be expanded in terms of spherical functions while the geometry of the system is best expressed in cylindrical coordinates with the $z$-axis along the fiber and the origin located at its center. Such an approximation becomes a problem when in-plane components of the heat flow are important. For materials with relatively low thermal properties, that component can be ignored.

In order to better understand heat propagation in fiber reinforced composites, simulations of the temperature response were made for cases where the fiber is more conductive/ diffusive than the matrix material and vice versa. Fig. 2.7 defines the parameters used in the simulations. A cylindrical coordinate system has its origin at the center of the fiber. A point $P$ has coordinates $(r, \theta)$. A heat source is located at a distance $r_{0}$ along the horizontal axis at the back of the fiber. It is assumed that the heat source is in the matrix of the composite material. The distance $r_{0}$ is always greater than the radius of the fiber $a$. The heat source is periodic with frequency $\omega$. The matrix has thermal conductivity $K_{I}$ and diffu- 
sivity $\alpha_{1}$, the fiber has properties $K_{2}$ and $\alpha_{2}$.

Simulations of the temperature fields due to the heat source and the scattering off the fiber were made for various material properties. Results are presented as surface plots.

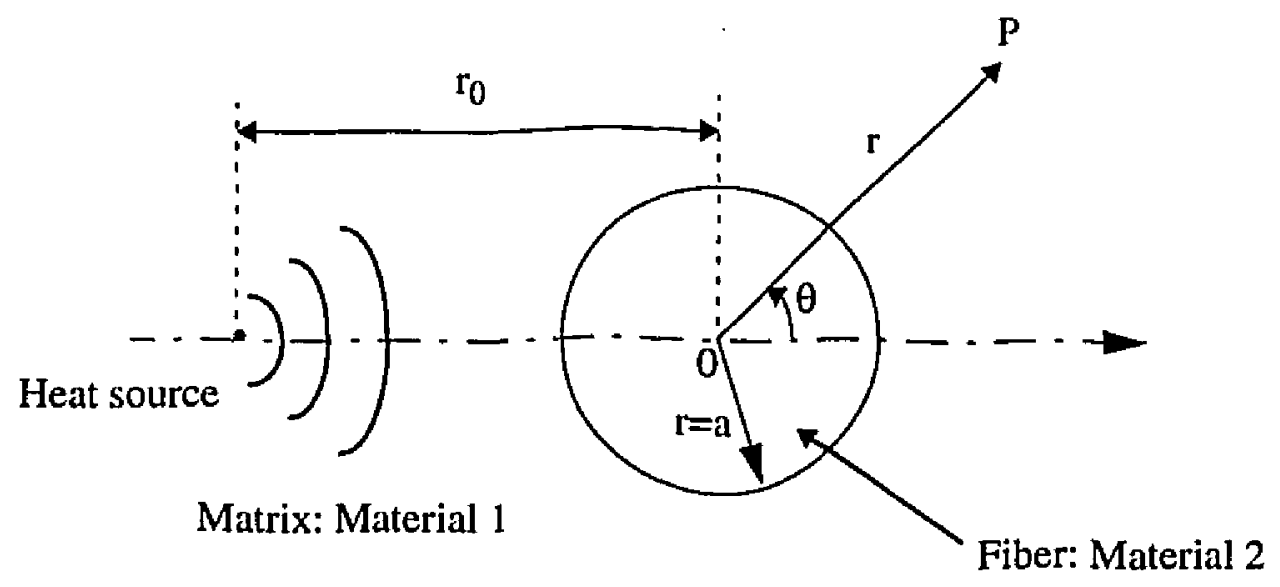

Fig. 2.7 Geometry and definitions used in simulations.

\section{a Temperature distribution due to the heat source only}

Fig. 2.8 shows a surface plot of the temperature field in an infinite solid due to a periodic point heat source located at $(0,0)$. The vertical axis is the temperature. The temperature is computed in the horizontal plane. As expected for an isotropic homogeneous material, Fig. 2.8 shows that the distribution is symmetric around the source. 


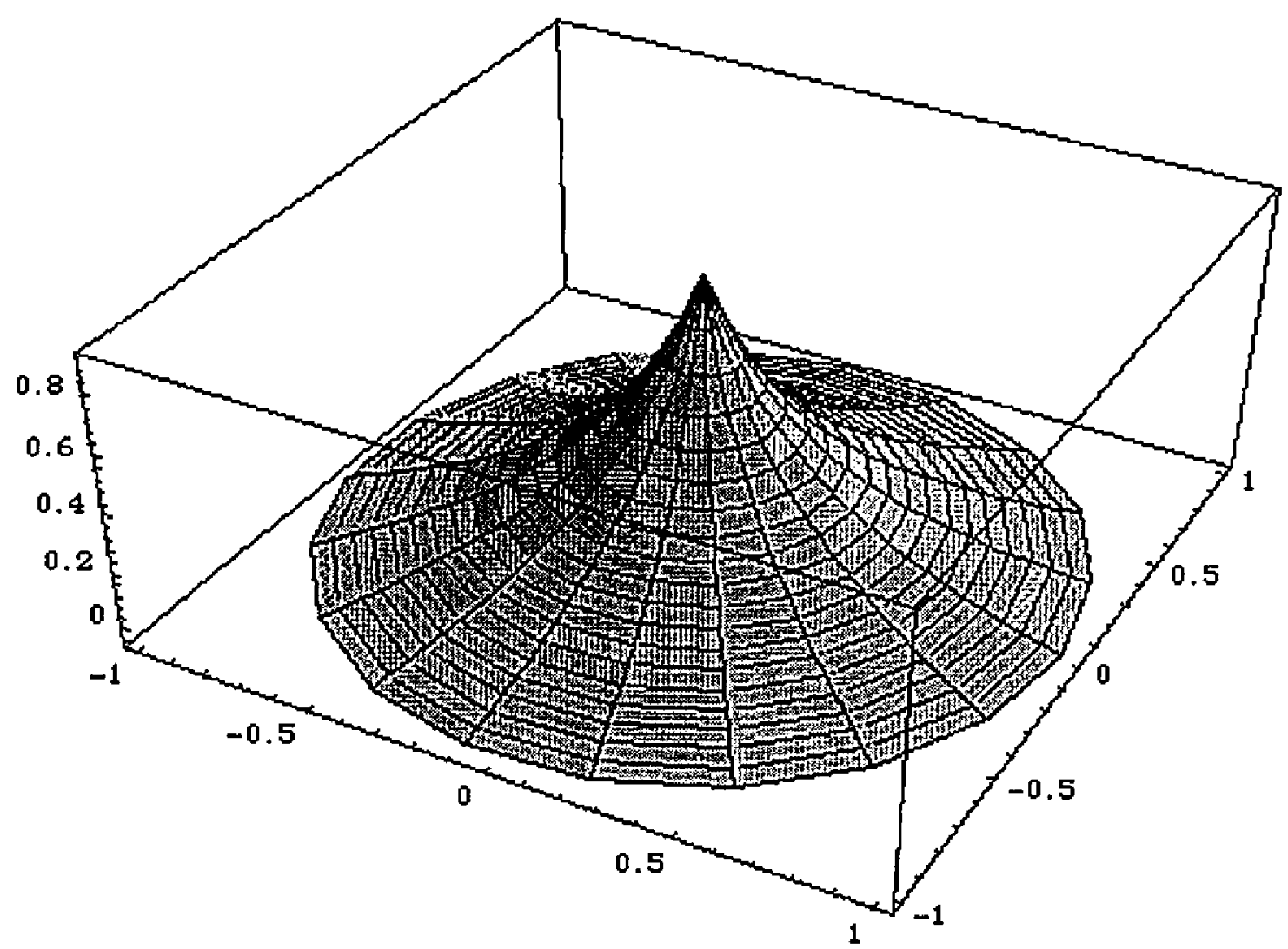

Fig. 2.8 Surface plot of the temperature distribution in the matrix due to the heat source only. The scaling factor on the vertical axis is $210^{-4}$. The horizontal axis are distances in terms of $\mathbf{r}_{0}$.

\section{b Temperature distribution due to the scattering off the fiber}

Fig. 2.9 and 2.10 show surface plots of the temperature distribution for the case where the fiber has thermal conductivity equal to zero. The arrow represents the axis along which the heat source and the center of the fiber are aligned. For such a case, heat diffuses from the source into the matrix and is blocked by the less conductive fiber. As heat accumulates in front of the fiber, the temperature locally increases. At the opposite side of the 
fiber, the temperature is lower than that of the surrounding matrix. The fiber acts as a heat shield. As seen in Fig. 2.10, the distribution is not antisymmetric. The amplitude of the thermal response due to the fiber is larger at the front than the back.

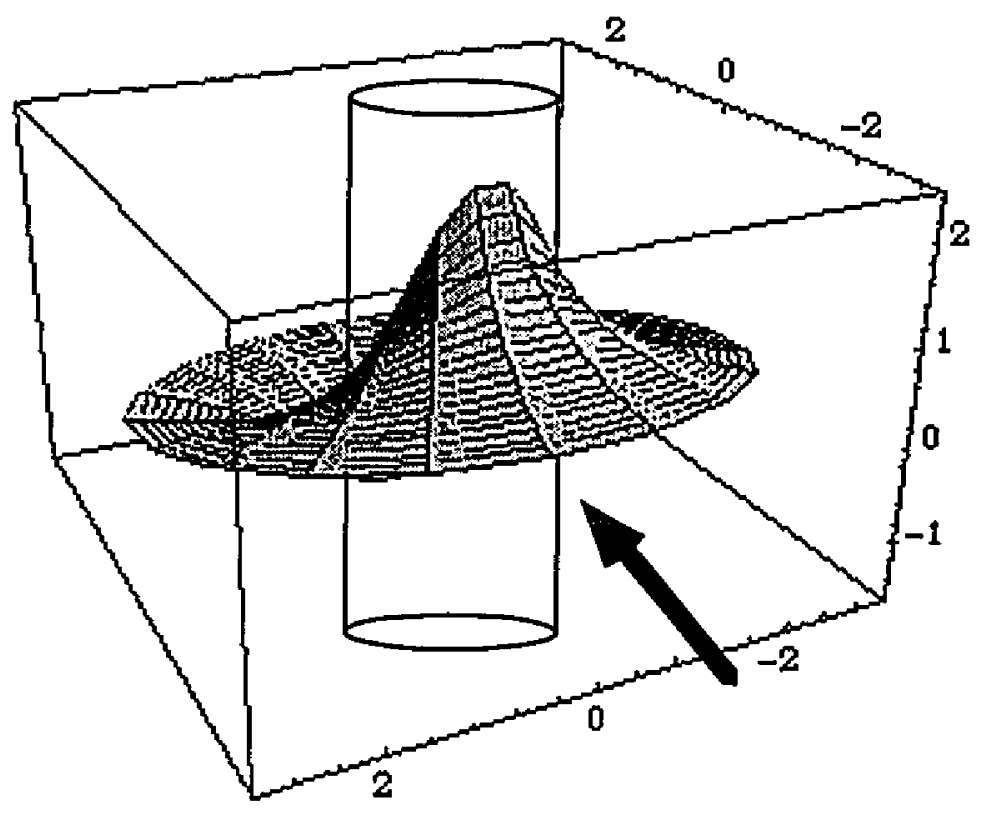

Fig. 2.9 Surface plot of the temperature distribution due to the scattering off a fiber less conductive/diffusive than the matrix. The fiber is indicated and has thermal conductivity equal to zero. The vertical axis represents temperature. The fiber has a radius of 75 $\mu \mathrm{m}$. The temperature field is represented inside a radius of $225 \mu \mathrm{m}$ around the fiber. The $\mathrm{X}-\mathrm{Y}$ dimensions are multiplied by $100 \mu \mathrm{m}$. The arrow indicate the direction of heat diffusion. 


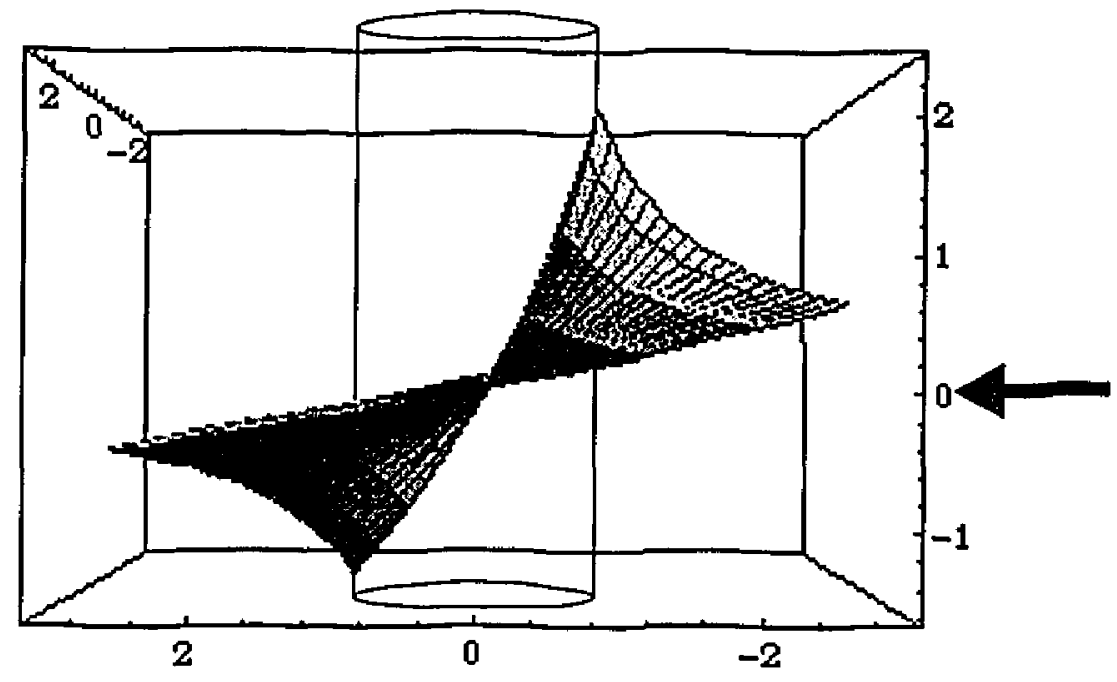

Fig. 2.10 Side view of the surface plot shown in Fig. 2.9. The fiber is also indicated.

Fig. 2.11 and 2.12 show surface plots of the temperature distribution for the case where the fiber is more conductive than the matrix. In this case, the fiber acts as a heat sink. As heat diffuses from the source toward the fiber, it is absorbed at a faster rate than in the surrounding matrix. As a result, the temperature in the matrix at the front of the fiber is lower. In the fiber, heat diffuses faster and, at its back, is blocked by the matrix, which acts as a heat shield. The temperature in the matrix at the opposite side of the source is higher than in the surrounding matrix. Fig. 2.12 shows a reverse situation of the case where the fiber is less conductive. The amplitude of the temperature drop on the side of the heat source is larger than the increase at the opposite side. 


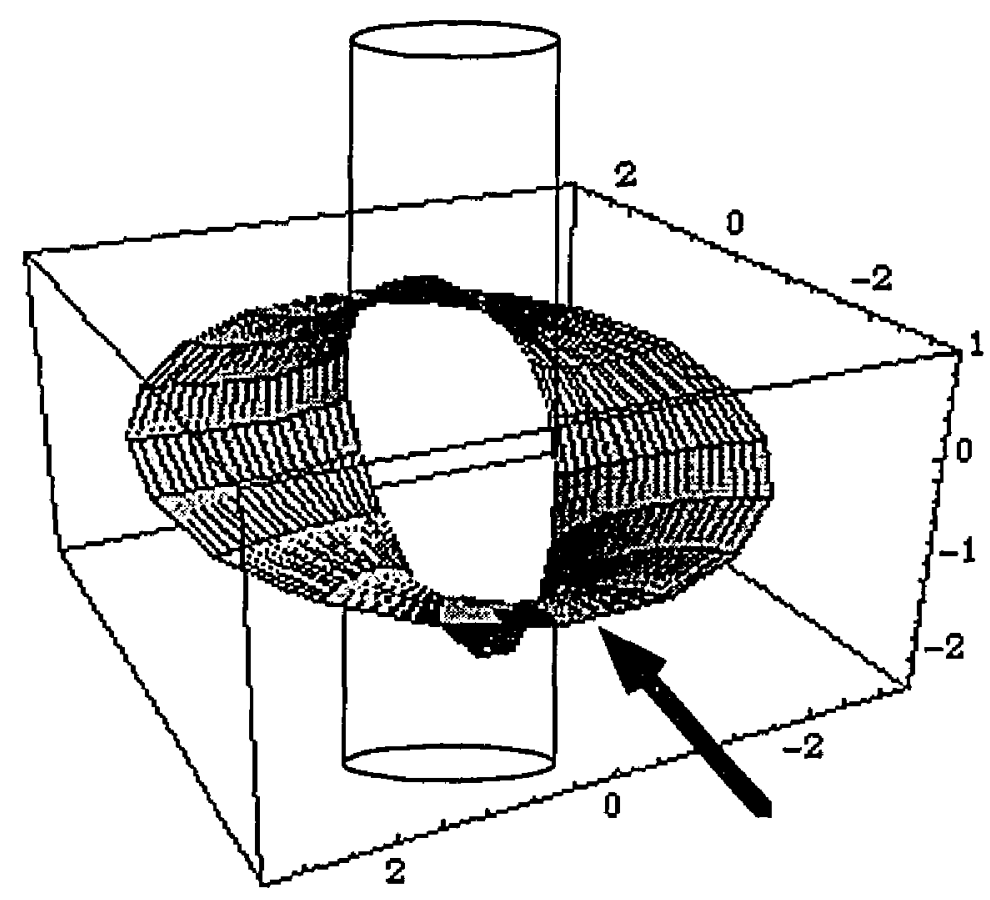

Fig. 2.11 Surface plot of the temperature distribution due to the scattering off a fiber. The fiber has higher thermal conductivity and diffusivity than the matrix. The fiber is also represented.

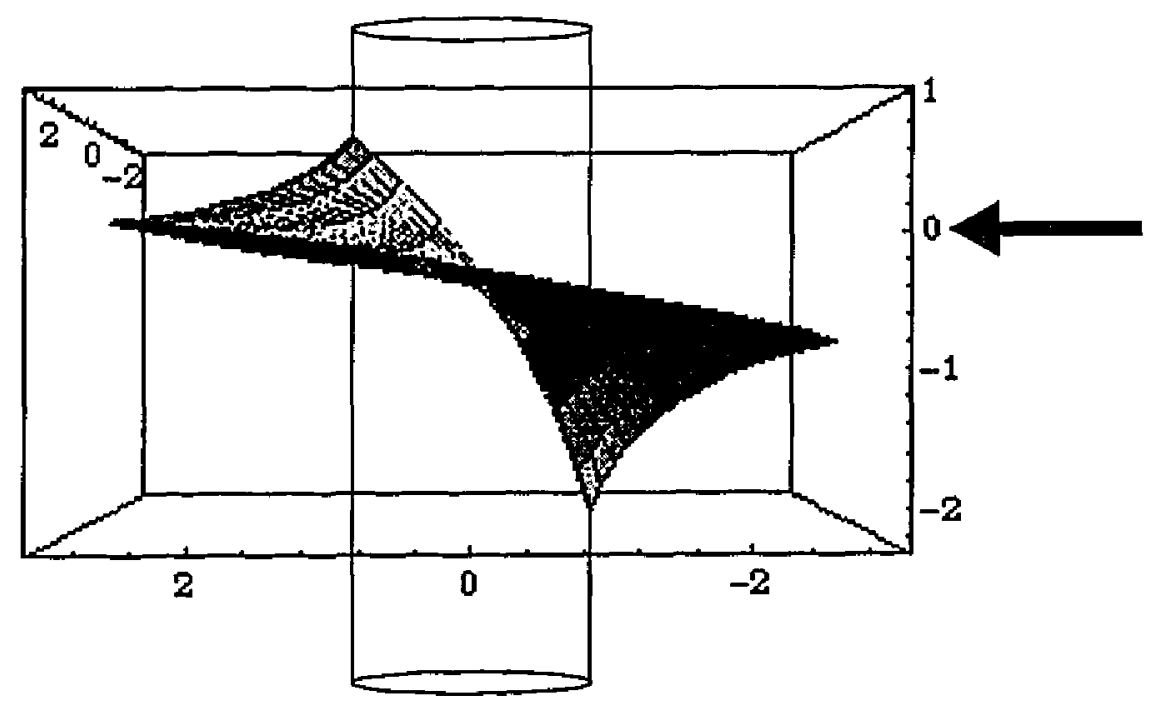

Fig. 2.12 Side view of the temperature distribution shown in Fig. 2.11. The fiber is 
also indicated.

\begin{tabular}{|l|r|r|r|r|}
\hline \multicolumn{1}{|c|}{ Figures } & $\begin{array}{c}\alpha_{1} \\
\left(10^{6} \mathrm{~m}^{2} / \mathrm{s}\right)\end{array}$ & $\begin{array}{c}\alpha_{2} \\
\left(10^{6} \mathrm{~m}^{2} / \mathrm{s}\right)\end{array}$ & $\begin{array}{c}K_{1} \\
(\mathrm{~W} / \mathrm{m} \mathrm{K})\end{array}$ & $\begin{array}{c}K_{2} \\
(\mathrm{~W} / \mathrm{m} \mathrm{K})\end{array}$ \\
\hline \hline 2.9 and 2.10 & 50 & 5 & 120 & 0 \\
\hline 2.11 and 2.12 & 0.5 & 1 & 1 & 10 \\
\hline
\end{tabular}

Table 2.3 Values for the parameter used in the simulations of Fig. 2.9, 2.10, 2.11 and 2.12. All simulations were made for an excitation frequency of $10 \mathrm{~Hz}$. Radii $a=150 \mu \mathrm{m}$ and $r_{0}=300 \mu \mathrm{m}$.

The simulations shown in Fig. 2.9 to 2.12 helped understand the propagation of heat in a composite material. But measurements described in chapter IV were made at the location of the heat source when it is moved slowly at the surface of the specimens in a direction perpendicular to the fibers. Fig. 2.13 and 2.14 show the predicted temperature profiles for such a configuration. Predicted profiles are displayed for different fiber diameters in Fig. 2.13 and for different fiber depths in Fig. 2.14. In all simulations, the fiber is less conductive than the surrounding matrix. The thermal conductivity of the fiber is set to zero.

In Fig. 2.13, the diameter of the fiber is changed and the depth is left constant. As expected, the model predicts that the amplitude increases with the diameter. No noticeable change in the shape of the temperature profiles is found for this range of parameters.

Fig. 2.14 illustrates the changes in thermal response when the depth of the fiber is changed. In this case, not only the amplitude changes dramatically but the shape is also altered. As the fiber is located closer to the source, the thermal response has a higher amplitude. At the same time, the peak is getting sharper whereas the temperature profiles for $y>0.0005 \mathrm{~m}$ are almost identical. This is characteristic of any diffusive process. The 
temperature distribution due to the scattered field is broader if the fiber is deeper into the matrix.

In Fig. 2.15, the depth and diameter of the fiber are left constant. The frequency of the excitation varies from 1 to $20 \mathrm{~Hz}$. The temperature profiles in the matrix along the horizontal axis reveal that an increase in frequency results in a decrease of the amplitude of the scattered field.

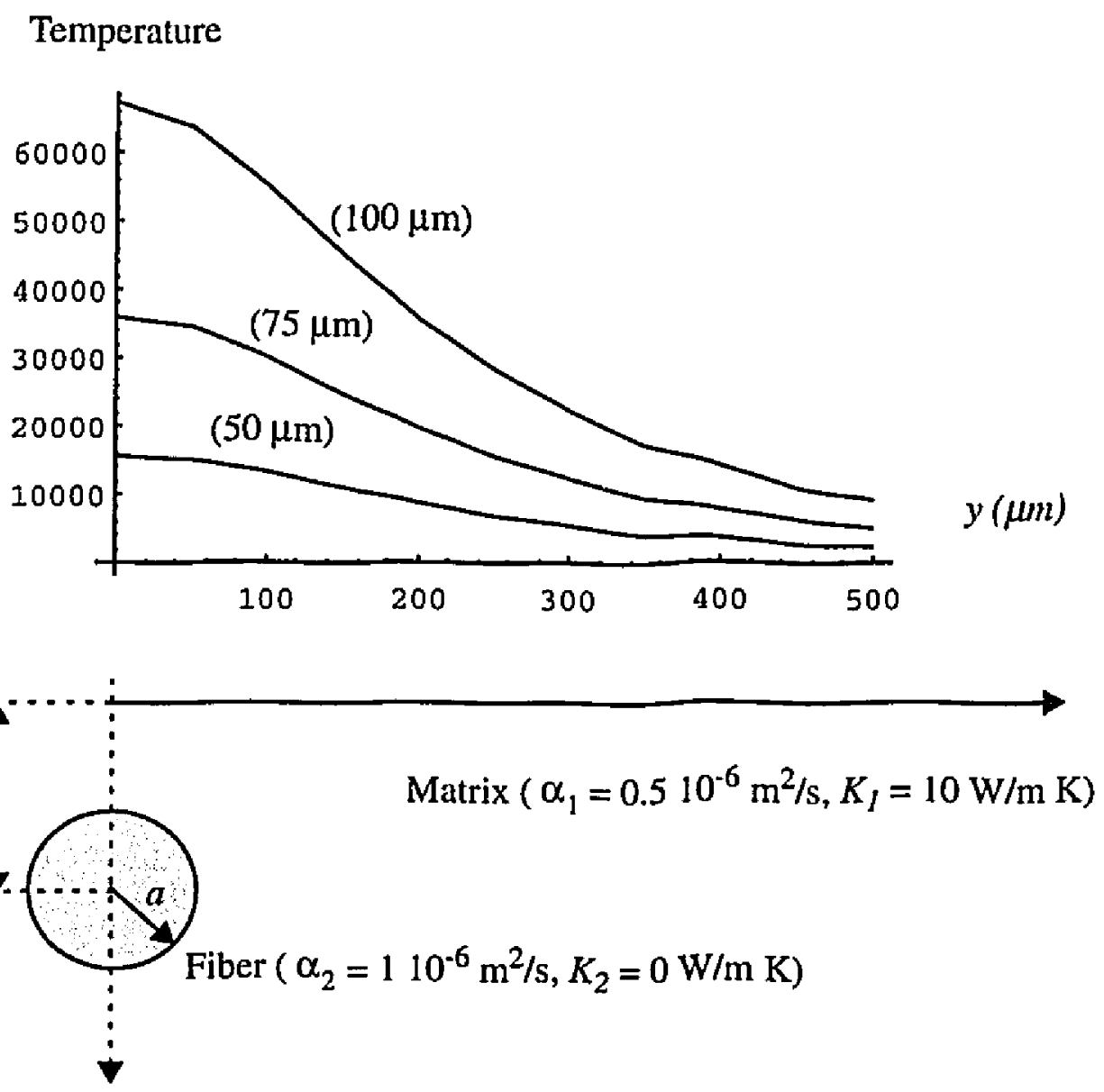

Fig 2.13 Simulation of the temperature profile as a function of $y$ for fiber diameters 50, 75 and $100 \mu \mathrm{m}$. The excitation frequency is $1 \mathrm{~Hz}$. Distance $r_{0}=225 \mu \mathrm{m}$. Units for temperature are arbitrary. 


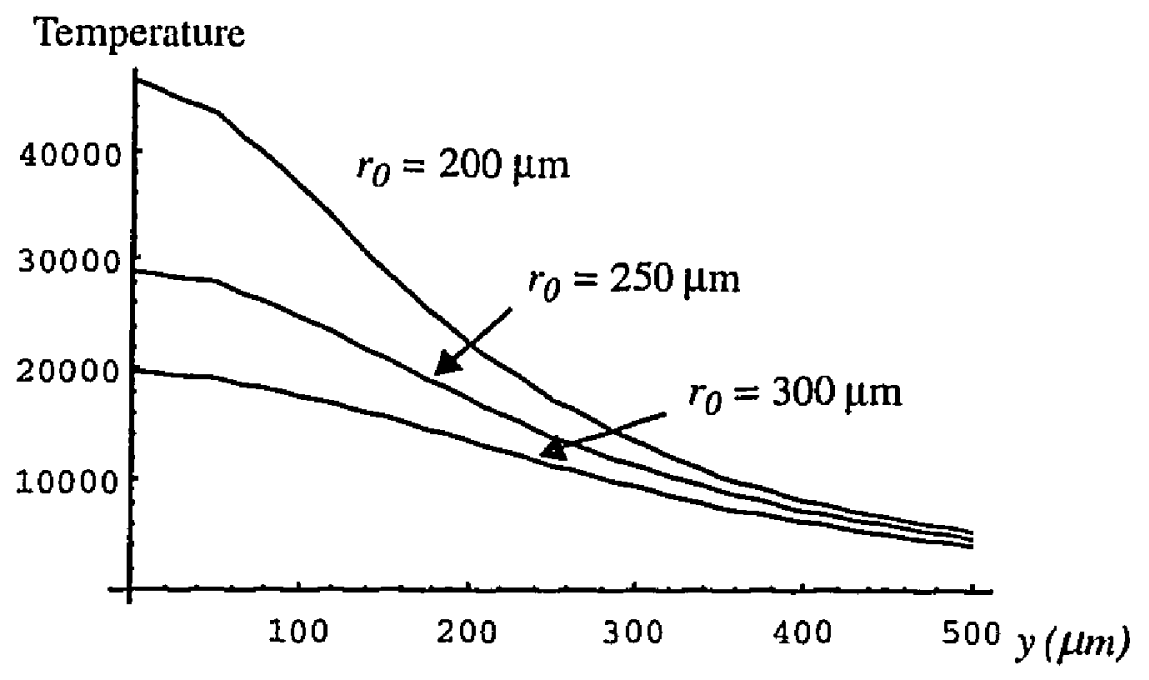

Fig 2.14 Simulation of the temperature profile as a function of $y$ for depths $r_{0}$ of 200, 250 and $300 \mu \mathrm{m}$. The excitation frequency is $1 \mathrm{~Hz}$. The thermal properties of the matrix and fiber are the same as the ones used in Fig. 2.13. Fiber radius is $a=75 \mu \mathrm{m}$. Units for temperature are arbitrary.

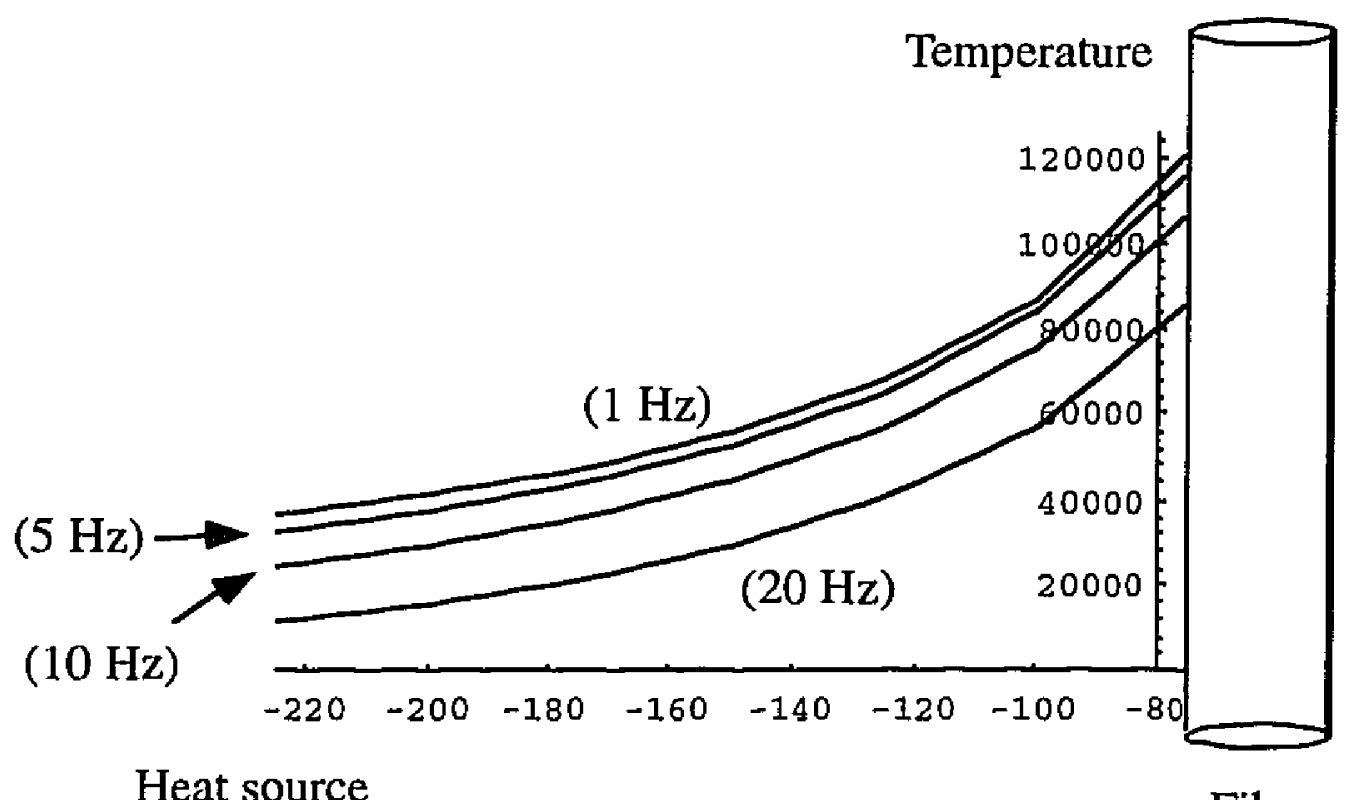

Heat source

Fiber

Fig 2.15 Simulation of the temperature profile as a function of depth for excitation frequencies ranging from 1 to $20 \mathrm{~Hz}$. The depth of the fiber is $r_{0}=150 \mu \mathrm{m}$. The fiber has thermal conductivity $K=0$ and radius is $a=75 \mu \mathrm{m}$. Units for temperature are arbitrary. 


\section{CHAPTER III}

\section{EXPERIMENTAL PROCEDURES AND APPARATUS, SPECIMENS STUDIED.}

Chapter II introduced the models used to predict the temperature responses of multilayer slabs and composite materials as a function of their geometries and thermal properties. Experimental data was taken on typical samples. This chapter describes the specimens studied and the experimental procedures and setups used to measure their thermal responses.

Depending on the type of specimen, two layer slabs, three layer slabs and fiber reinforced composite materials, different data acquisition setup configurations were used. The principles of the experiment remain the same: a radiant heat source excites the specimen and the temperature response at the surface is monitored. The same detector, an infrared focused point detector, was used in all measurements. The detector is described at the beginning of the chapter. Then, a presentation of the apparatus and the specimens follows.

\section{A Flat multilayered geometry}

\section{Experimental apparatus}

All experiments in this work involved the measurement of the temperature time history 
at the surface of the specimens. In the case of two layer slabs, the temperature was monitored while the specimens were periodically excited and in the case of three layer slabs, following a short duration heat pulse. The detection method was preferably noncontacting with a good time resolution of the temperature variations. A convenient solution was to use a commercially available infrared imager as a focused point detector. Since the point detection option was not offered on the equipment available, the infrared imager was modified.

\section{a Modification of the infrared imager}

The IR imager used is an Inframetrics model 525, a small field instrument designed to produce a video output signal of the thermal patterns it is viewing. The imaging system is linked to an electronics/control/power supply unit.

The IR radiation emitted from the specimens is detected by a liquid Nitrogen cooled Mercury-Cadmium-Telluride (HgCdTe) detector. For conventional operation of the imager, the output of the detector is converted to video signal. An ambient temperature chopper is viewed by the detector at a rate of $60 \mathrm{~Hz}$ to serve as a temperature reference. The solid angle subtended by the detector is approximately $10^{-6}$ steradian. An image of the area of interest is formed by scanning the field of view of the detector with two low inertia mirrors mounted on galvanometers. The vertical mirror scans the area at a rate of $60 \mathrm{~Hz}$. The horizontal mirror, mounted at a $90^{\circ}$ angle from the vertical mirror, scans the area at a rate of $3933 \mathrm{~Hz}$. The two mirrors are synchronized to enable generation of an image.

The conventional operation of the system allows a mode where the vertical mirror is 
held stationary. The position of the mirror is controlled by varying a DC potential from the control unit. With the horizontal mirror running, a line scan is performed. The galvanometer controlling the horizontal mirror is $\mathrm{AC}$ driven and, under conventional operation, its scanning rate cannot be adjusted. Fig. 3.1 shows the optical schematics of the imager.

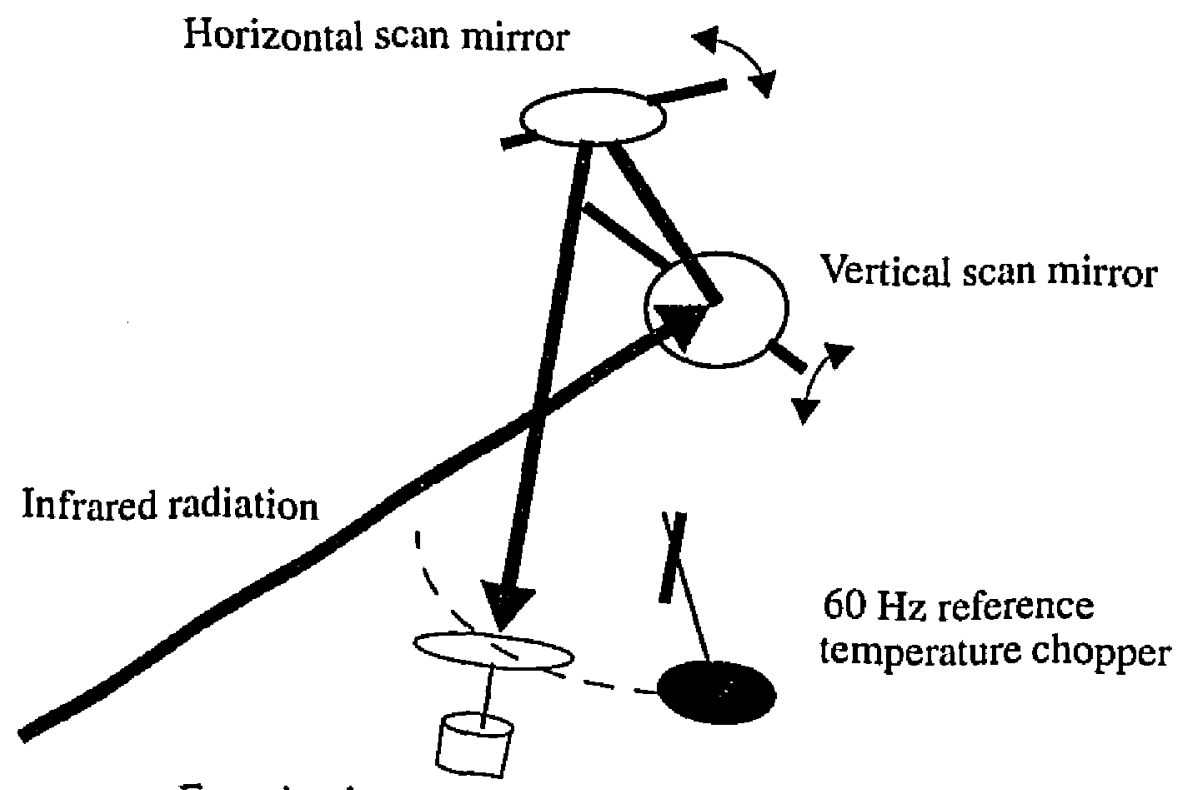

Focusing lens

and detector

Fig. 3.1 Optical schematic of infrared imager.

The infrared imager was modified to permit direct control of the scanning mirrors and to access the detector signal. This allowed to measure the changes in surface temperature at a point as a function of time with high temporal resolution. The ambient temperature chopper was disabled to obtain continuous readings. The modifications were achieved by inserting a control unit between the imager and the Inframetrics video controller so that no irreversible hardware changes were necessary. 


\section{b Data acquisition for two layer specimens}

A block diagram of the experimental setup for the measurement of thermal properties on two layer samples is shown in Fig. 3.2. A thermal stimulus is provided by an IonArgon laser. Since the Laser emits in the green region of the visible spectrum only, the thermal signature detected by the infrared detector does not contain a reffective component from the heat source. The beam is controlled by an acousto-optic modulator which allows the generation of a periodic square pulse signal over a wide range of frequencies (continuous to $50 \mathrm{MHz}$ ). A lens system expands the beam spot at the surface of the specimens to approximate a plane heat deposit around the region of detection.

The detector preamplifier has a lower frequency cut off at $10 \mathrm{~Hz}$. To enable measurements of the order of one second or more, the radiation into the detector is modulated by a mechanical chopper. The signal from the detector is rectified by a lock-in amplifier and then digitized by an oscilloscope. A low frequency square signal is used for laser modulation and the trigger pulse for the oscilloscope. A much higher frequency drives the mechanical chopper modulating radiation into the detector. The signal is averaged to further improve the signal to noise ratio. A computer controls the oscilloscope and stores the digitized signal. 


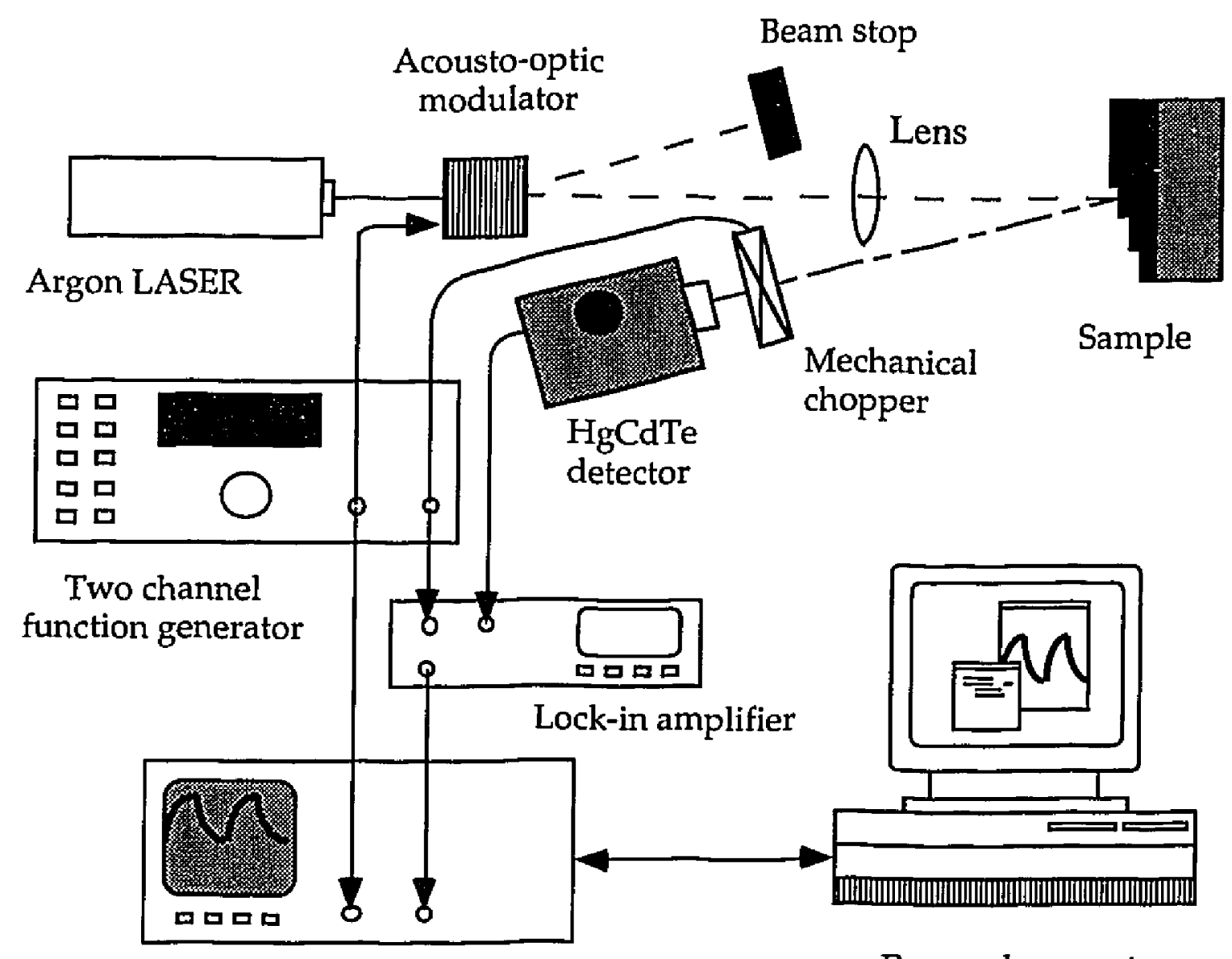

Oscilloscope

Personal computer

Fig. 3.2 Experimental setup with Laser and point infrared detector for data acquisition on two layer specimens.

\section{c Data acquisition for three layer specimens}

Fig. 3.3 shows the experimental setup used for the measurement of thermal properties on three layer samples. A thermal stimulus is provided by a flash tube behind the sample and the temperature variations are monitored on the front surface. The detection is performed as for the two layer samples, with radiation from the specimen chopped, rectified by a lock-in amplifier and finally digitized by an oscilloscope. The output signal of a func- 
tion generator is used as a driver for the chopper and as a reference for the lock-in amplifier. The pulse triggering the flash lamp is also used to trigger the data acquisition.

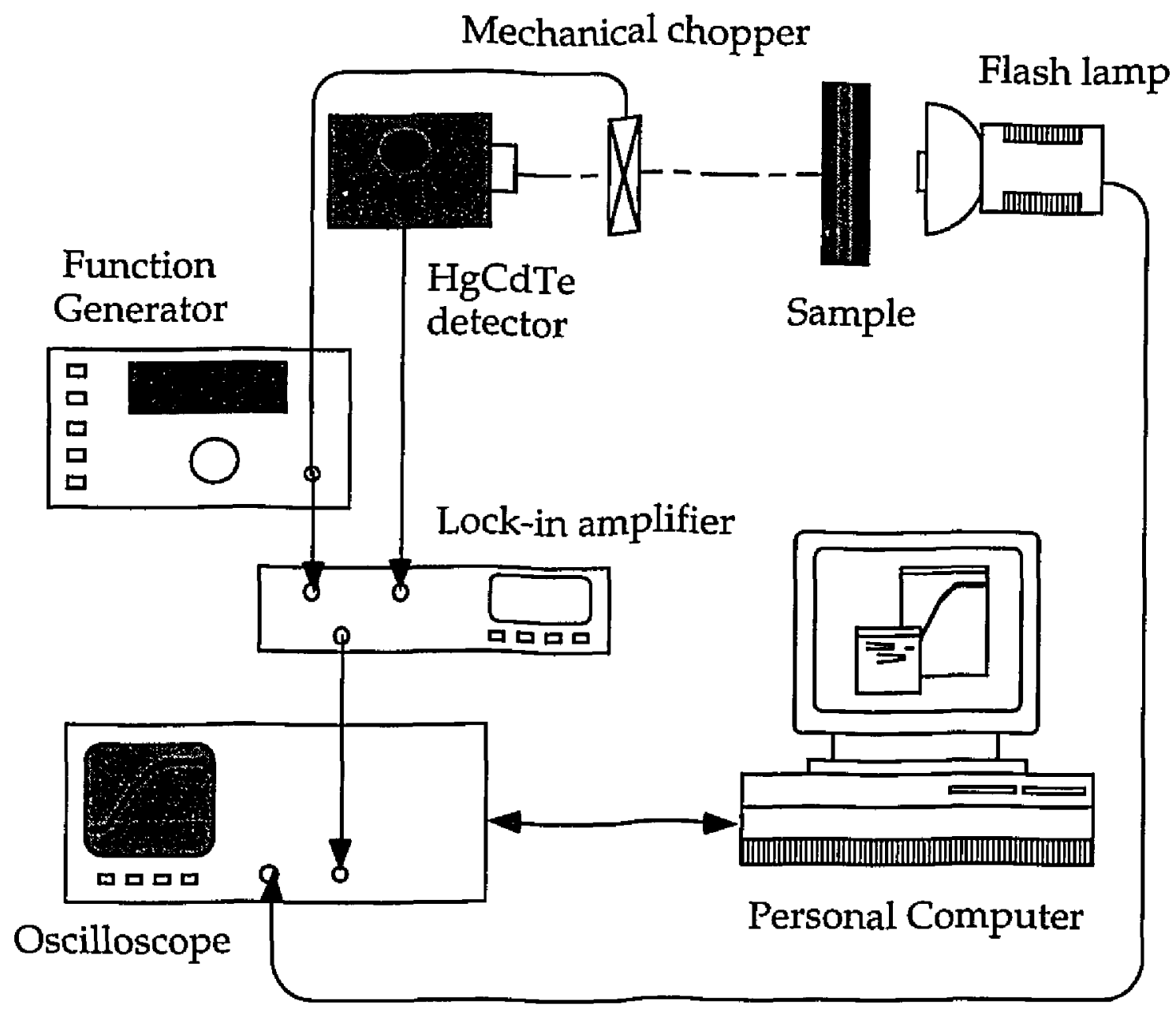

Fig. 3.3 Experimental setup with flash lamp and point infrared detector for data acquisition on three layer specimens.

\section{Samples, preparation, geometry}

\section{a Two layer specimens}

Data was taken for three samples composed of $1.016 \mathrm{~mm}$ thick 2024-T3 aluminum plates coated with different thicknesses of black paint. The coating thicknesses were 
$0.074,0.0194$ and $0.0324 \mathrm{~mm}$.

\section{b Three layer specimens}

Fig. 3.4 shows the geometry of the specimens for simulated aluminum corrosion. The outer $1.016 \mathrm{~mm}$ thick 2024-T3 aluminum slabs are held together by 8 bolts. In Fig. 3.4, only the one on the upper left corner is represented. The spacing between the slabs is created by a plexiglass insert with a square shaped hole at the center. The material to be characterized is contained between the aluminum layers and, on the sides, by the plexiglass. The inner layer, composed of $\mathrm{Al}(\mathrm{OH})_{3}$ powder, is $0.5 \mathrm{~mm}$ thick. In order to increase surface emissivity, a thin layer of Sight Black ${ }^{\mathrm{TM}}$ was sprayed on the surface of all the samples. Increasing surface emissivity allows to maximize heat absorption by the specimens and reduces infrared reflections. The black layer deposited at the surface has a negligible influence on the thermal response of the multilayer structures. Typical times for the experiments were of the order of the second, which is much longer than the transient response times for the thin Sight Black ${ }^{\mathrm{TM}}$ coating.

Fig. 3.5 shows the geometry for the specimens with inner air and glycerin layers. The outer layers, two $1.016 \mathrm{~mm}$ thick 2024-T3 aluminum flat plates, were bonded together with 5-minute ${ }^{\mathrm{TM}}$ epoxy. A U-shaped plexiglass insert was used to create a constant spacing in the central region of the specimens. At the measurement points, the thickness is $0.52 \mathrm{~mm}$ for both air and glycerin. The specimen filled with glycerin was put in a vacuum chamber and exposed to low pressures for 8 hours to remove any residual air bubbles. The two sides of both samples were prepared to increase surface emissivity.

A specimen was made with an inner layer composed of epoxy resin. Two $1.016 \mathrm{~mm}$ 
thick 2024-T3 aluminum slabs were bonded together. After cure, the thickness of the epoxy layer was $0.308 \mathrm{~mm}$ at the measurement points. The two sides of the bonded structure were also coated to increase emissivity.

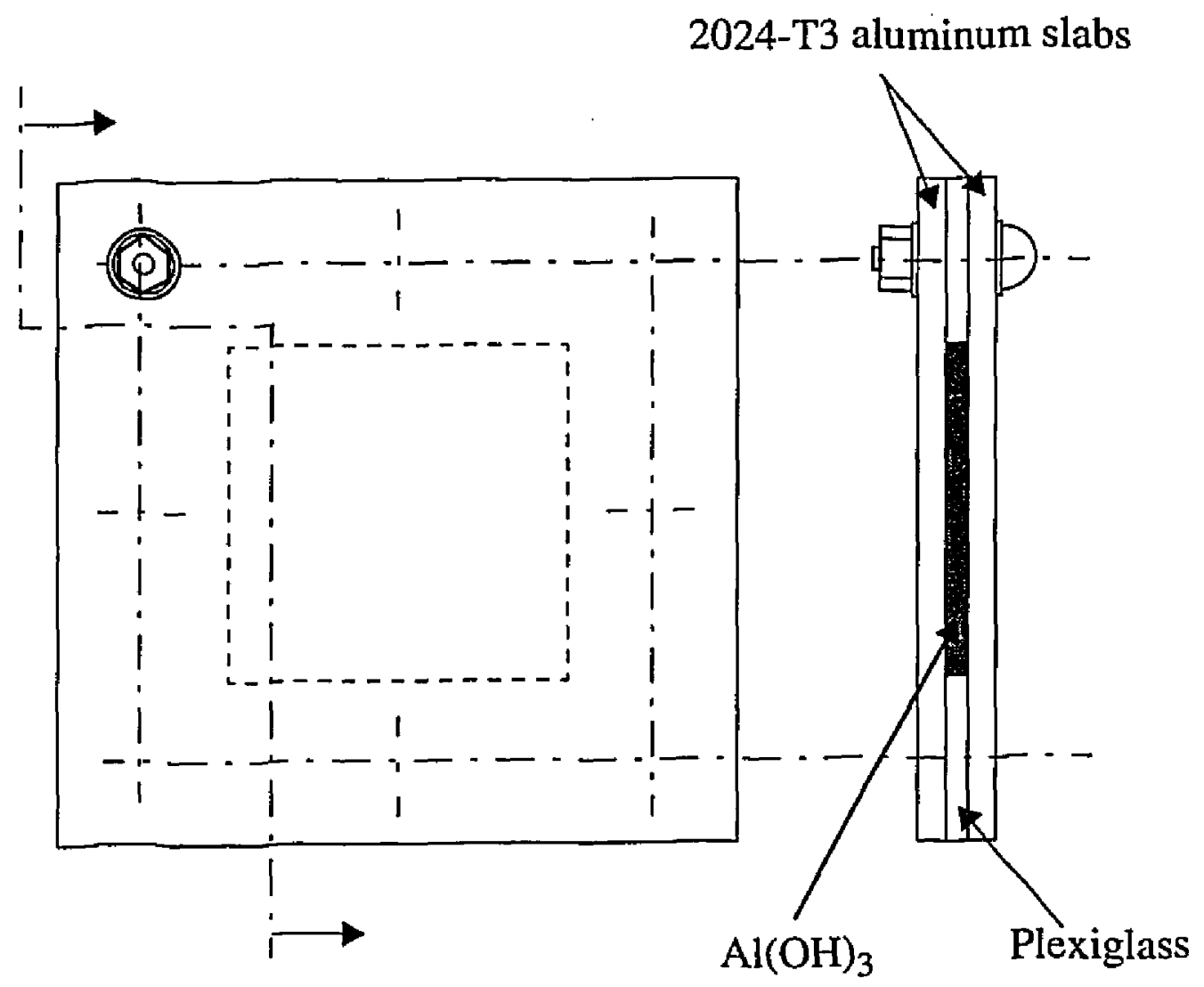

Fig. 3.4 Geometry of three layered specimen with simulated corrosion. The outside dimensions are $76.2 \mathrm{~mm} \times 76.2 \mathrm{~mm}$. The region of interest in the center is $38.1 \mathrm{~mm} x$ $38.1 \mathrm{~mm}$. 
Region of interest
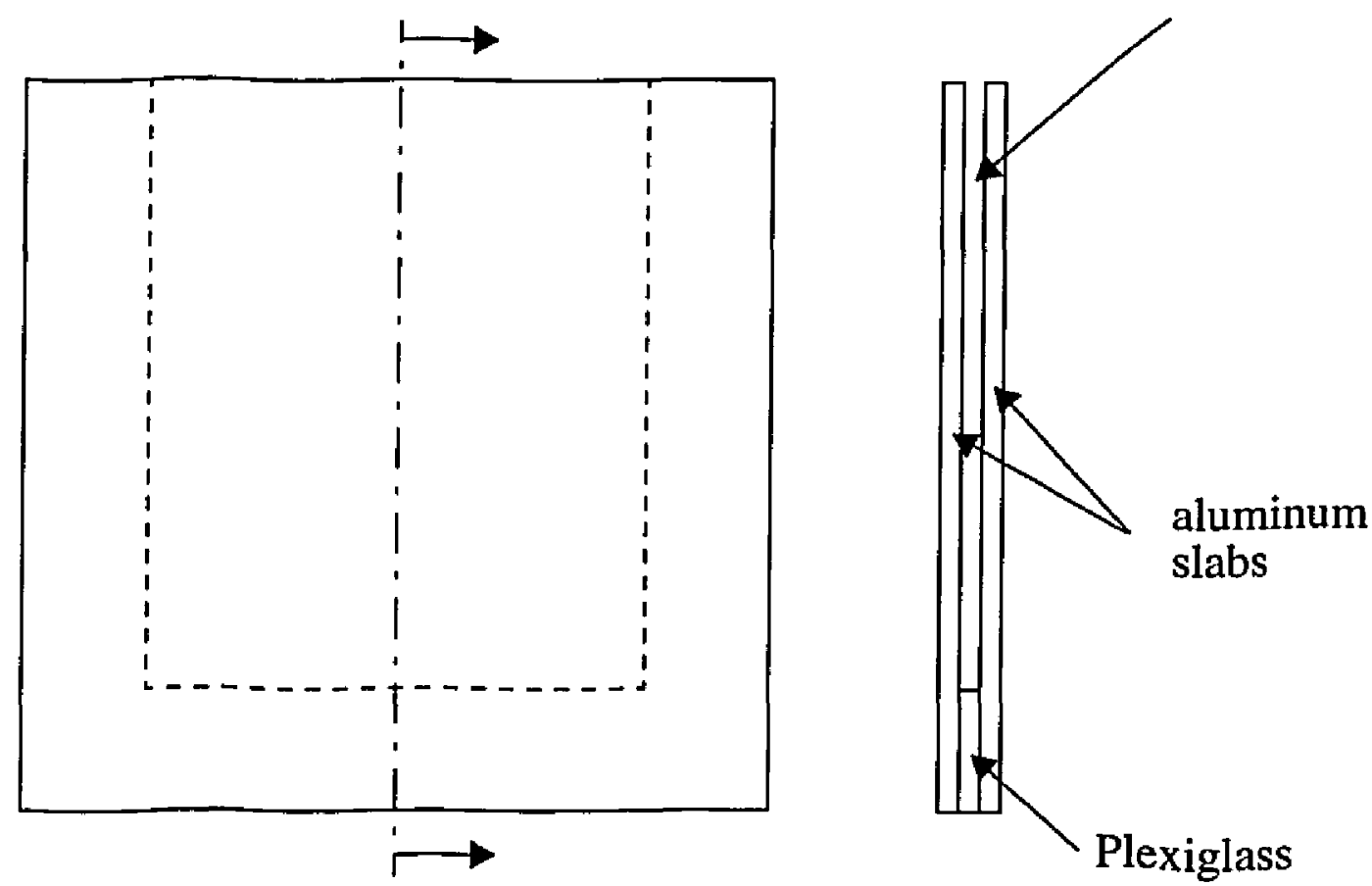

Fig. 3.5 Geometry of three layered specimens with air and glycerin. The outside dimensions are $76.2 \mathrm{~mm} \times 76.2 \mathrm{~mm}$. The region of interest is $50.8 \mathrm{~mm} \times 63.5 \mathrm{~mm}$. The region of interest in the center is filled with glycerin or let empty for the inner air layer specimen.

\section{B Fiber reinforced composite materials}

\section{Principle of experiment}

Fig. 3.6 shows the principle of the experiment for the characterization of composite materials. The interest is to image the thermal response at the surface of composite materials. The technique used in this case consists in rastering a heat source across the scanned area. The temperature profile as a function of time is obtained at each point to form an image. In order to maximize the detector signal, the measurements are made at the posi- 
tion where heat is deposited. The specimen moves in a plane perpendicular to the direction of detection and heat deposit. This simplifies the alignment process, compared with a stationary specimen and a rastered heat source and detector.

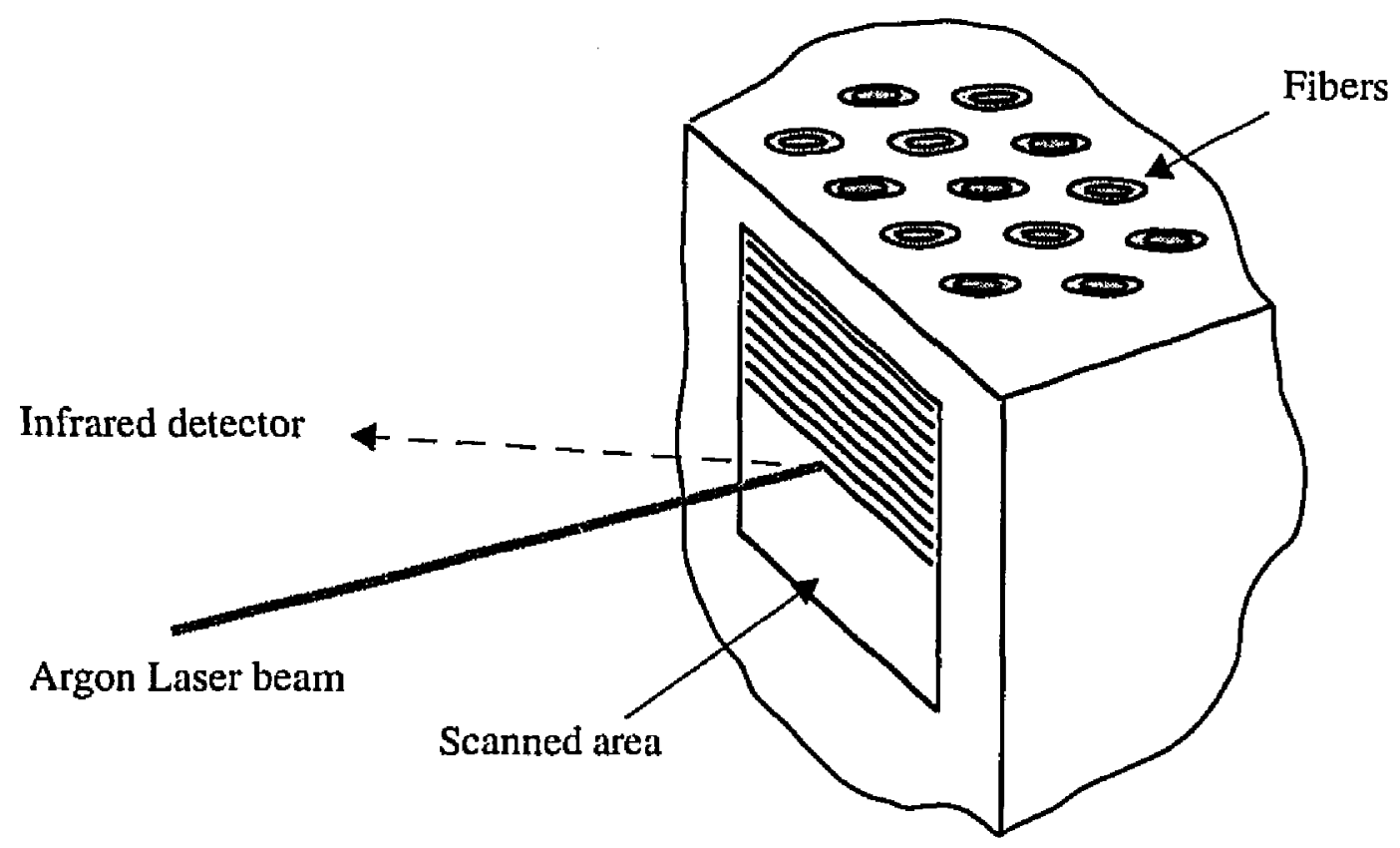

Fig. 3.6 Principle of the experiment for characterization of composite materials.

\section{Samples, geometry}

\section{a Fabricated Aluminium sample}

An aluminum flat plate was prepared to simulate the case of total disbonding between matrix and fiber. A hole was drilled parallel to a surface to simulate the disbonded fiber. Fig. 3.7 shows the geometry. 


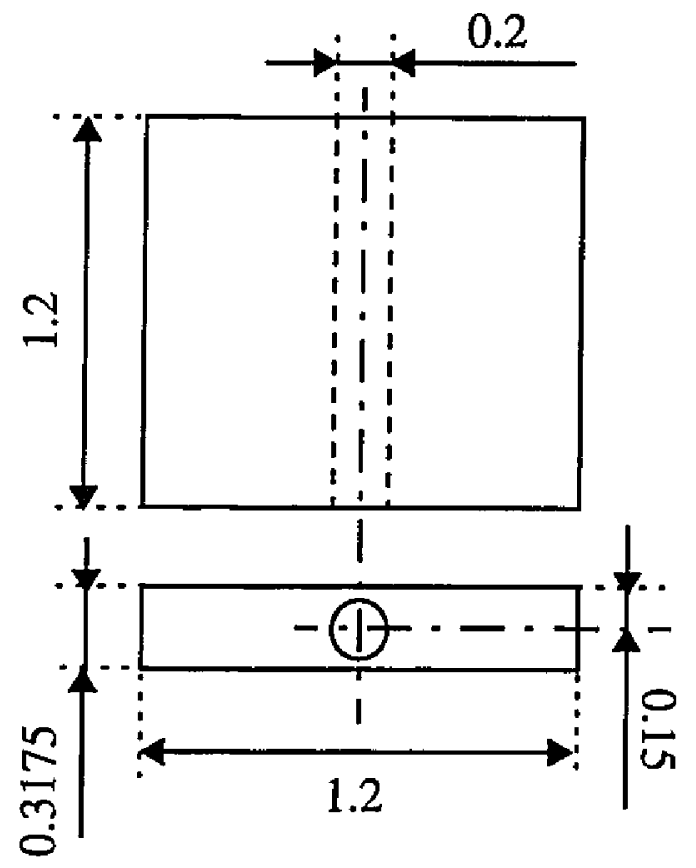

Fig. 3.7 Geometry of aluminum specimen. The dimensions are in mm.

\section{b Reaction bonded Silicon Nitride/SiC fibers composite specimens}

The two specimens used consisted of Reaction Bonded Silicon Nitride (RBSN) com-

posite materials with SiC fibers. Fig. 3.8 shows their geometry. The fibers were measured to have a diameter of $0.150 \mathrm{~mm}$. Specimen RBSN1 was the original and RBSN2 was degraded by heat treatment to induce damage. 


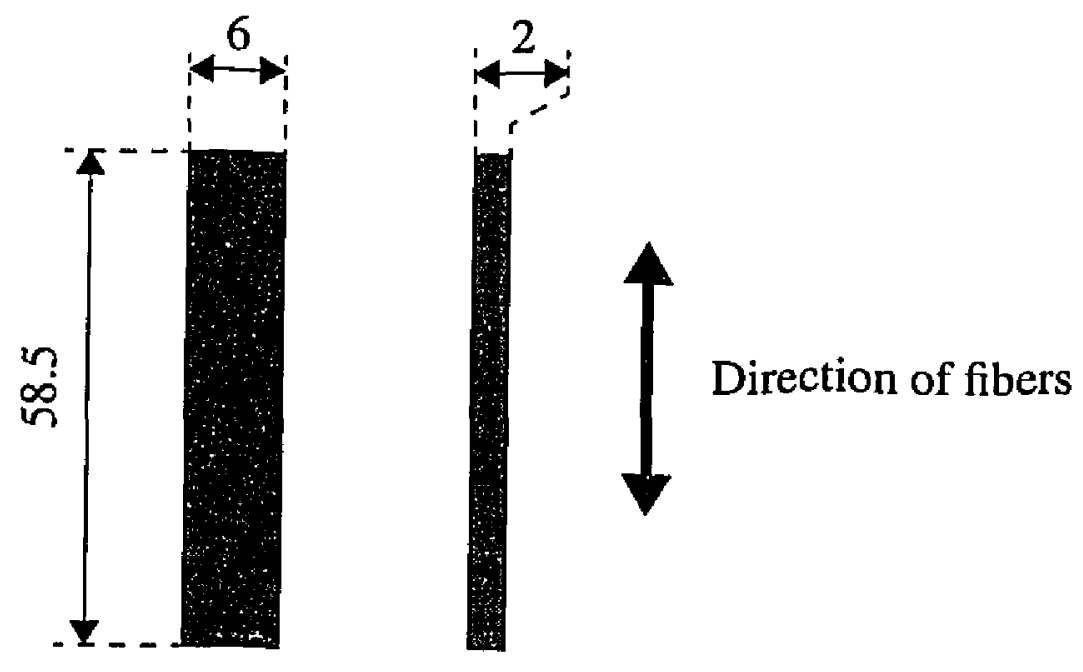

Fig. 3.8 Geometry of RBSN composite specimens. Dimensions are in mm.

\section{Experimental apparatus}

Fig. 3.9 shows the experimental setup used for the measurement of thermal properties on fiber reinforced composite materials. A thermal stimulus is provided by an Ion-Argon laser at the front surface of a sample. A laser beam is chopped at a desired frequency by an acousto-optic modulator. The temperature variations are monitored by an infrared detector at the point where heat is deposited. The signal is digitized by an oscilloscope. The specimen is mounted on a 2-axis translation stage which allows horizontal and vertical motion of the specimen with respect to the laser beam. Measurements are repeated at each new position in order to form a picture. A computer controls the data acquisition and the 2-axis translation stage. The temperature time history at each pixel is stored in the computer. 


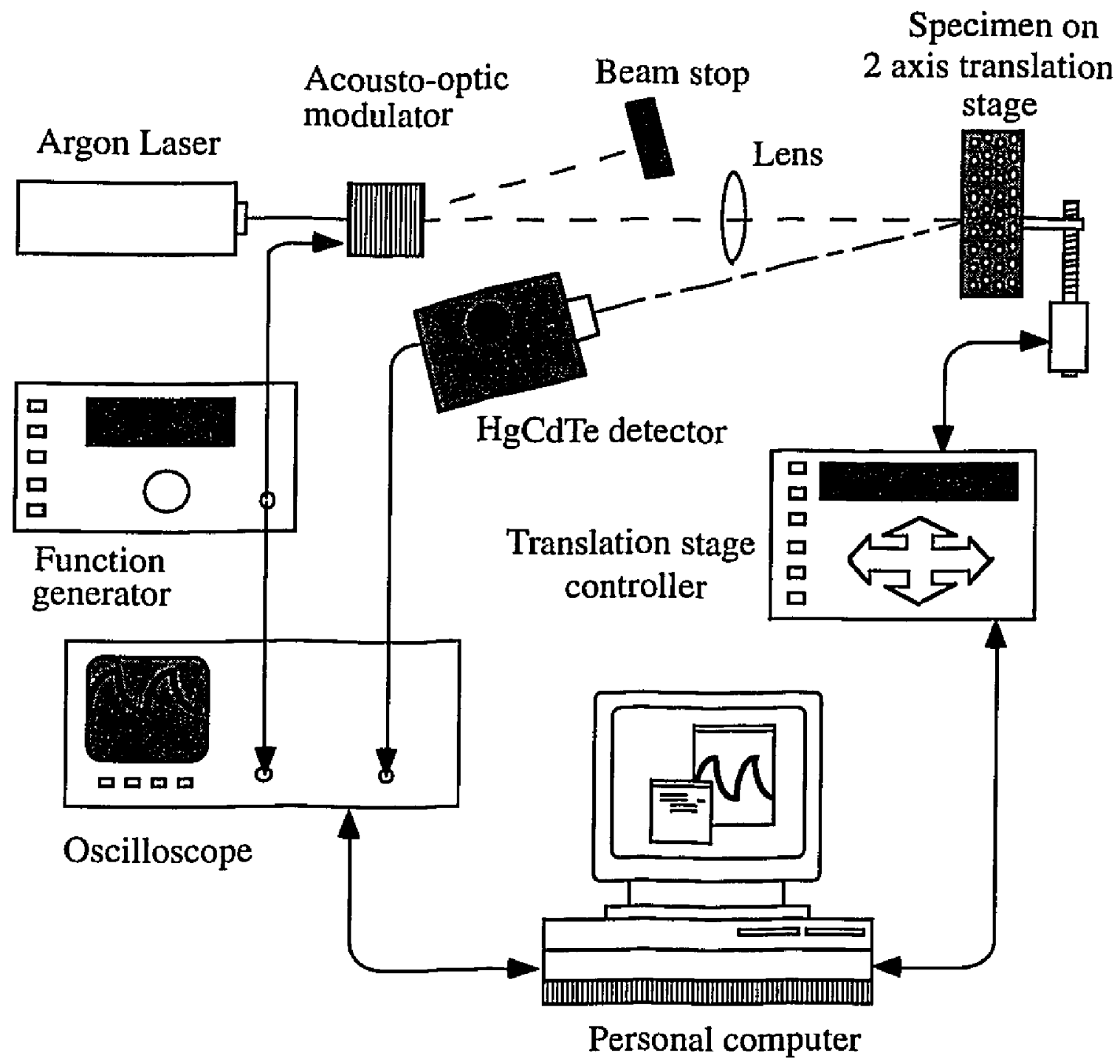

Fig. 3.9 Experimental setup for data acquisition on composite materials. 


\section{CHAPTER IV EXPERIMENTAL RESULTS}

Chapter II introduces theories of the propagation of heat in solids with specific geometries: multilayered structures and fiber reinforced composite materials. Given their thermal properties and their geometries, it is possible to predict the temperature response in time of the heated specimens. Chapter III describes the specimens to be characterized and the experimental setups to perform the measurements.

This chapter is an overview of these measurements for each type of sample. In the first section, examples of time resolved temperature responses are given for two and three layer structures. Also, other measurements using X-ray techniques and an infrared imager complement the study. Some qualitative information is extracted from these experimental results. In the second part of this chapter, time domain and Fourier transform images of fiber reinforced composite materials are shown.

\section{A Temperature profiles for flat multilayer structures}

\section{Two layer samples}

Data was taken for the three two layer samples described in chapter III for excitation frequencies ranging from 0.3 to $10 \mathrm{~Hz}$. Fig. $4.1,4.2,4.3$ and 4.4 show typical time-temperature profiles for these samples. Fig. 4.1 and 4.2 show results for $2 \mathrm{~Hz}$, Fig. 4.3 for 5 $\mathrm{Hz}$ and Fig. 4.4 for $0.3 \mathrm{~Hz}$. To improve visualization of shape variations, the graphed tem- 
perature responses are normalized in Fig. 4.2 and 4.3 by substracting the minimum in the data and then dividing by the maximum data values. The amplitude of the thermal responses can only be used for quantitative characterization when surface emissivity and amount of heat deposited are known. By normalizing, the emissivity and heat flux variations for different samples are removed.

For an excitation frequency of $2 \mathrm{~Hz}$, the three temperature responses can easily be differentiated. Sample 1, with the thinnest paint coating, has the fastest rise time. When heat is applied, the surface temperature rises quickly for approximately $20 \mathrm{~ms}$. Then, the increase in temperature slows down to a constant slope. When heat is applied for longer periods, the surface temperature increases steadily until the convection losses are no longer negligible. Fig 4.4 illustrates the case for sample 2 with a low excitation frequency resulting in a steady rise in surface temperature after approximately $0.5 \mathrm{~s}$. In Fig. 4.2, samples 2 and 3 exhibit a slower initial rise time in surface temperature after heat is applied. The variations in thickness coating are responsible for the variations in the initial responses of the specimens.

For heat applied to the surface of a two layered system, the time history temperature response can be divided into two regions. The initial thermal response is dominated by the first layer. When a significant portion of the heat begins to diffuse into the second layer, the response becomes that of the composite structure. If the first layer is thermally thin, the initial phase is short and the thermal response of the structure is quickly dominated by the second layer. The structure approximates a single layer with thickness and thermal properties of the second layer.

For an excitation frequency of $5 \mathrm{~Hz}$, little difference can be seen between the thermal 
responses for samples 2 and 3 . This is because the coating dominates the response at early times. This demonstrates the need for tailoring the frequency of the excitation to insure the response is a result composite of the two layers.

\section{Thermal response}
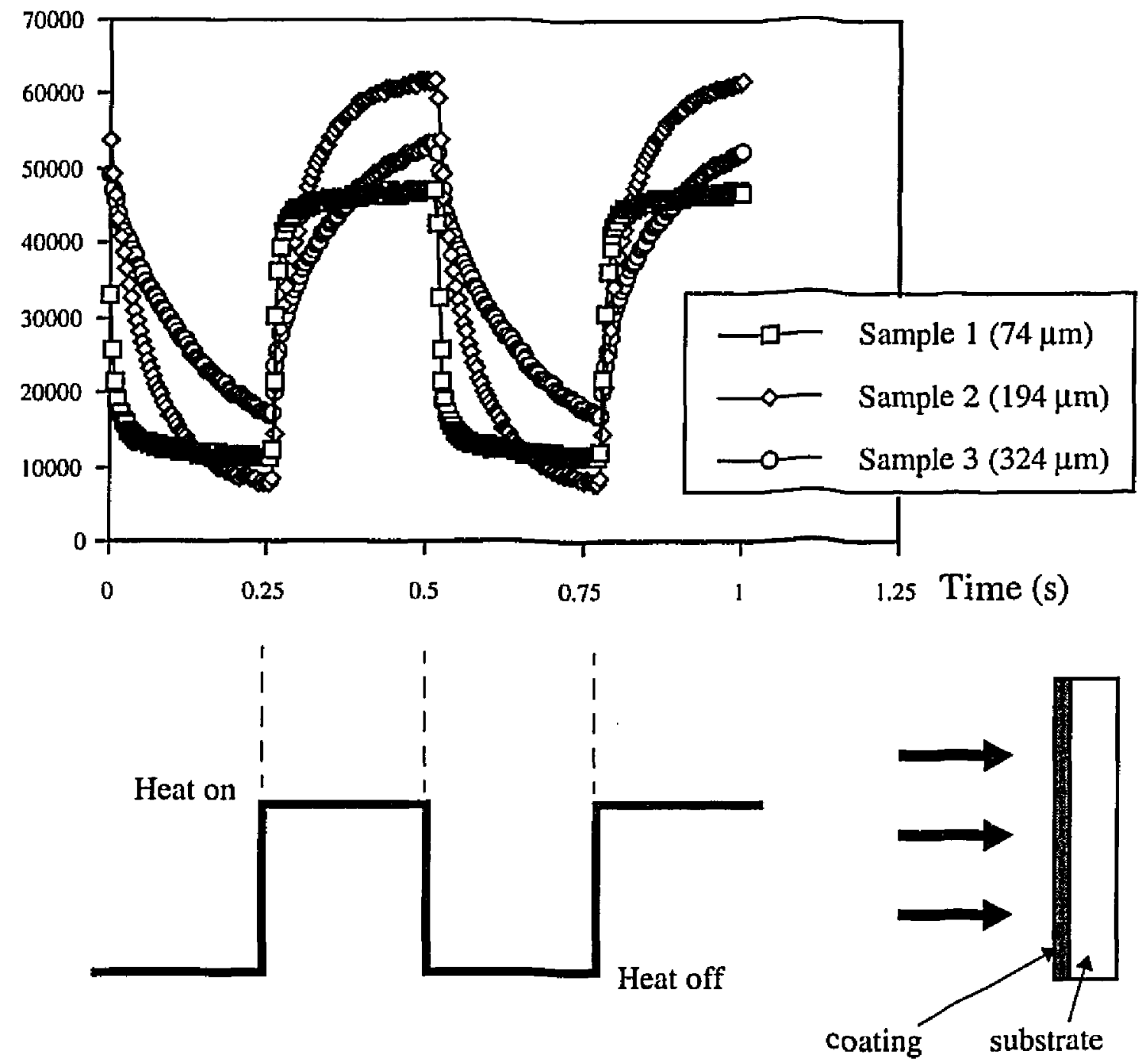

Fig 4.1 Example of raw data for temperature response of two layer specimens for an excitation frequency of $2 \mathrm{~Hz}$. Units for the vertical axis are arbitrary. The signal corresponding to the sample 1 has the largest amplitude. For convenience of the data acquisition and representation, it was divided by a factor 2 . The heat cycles are also represented. 


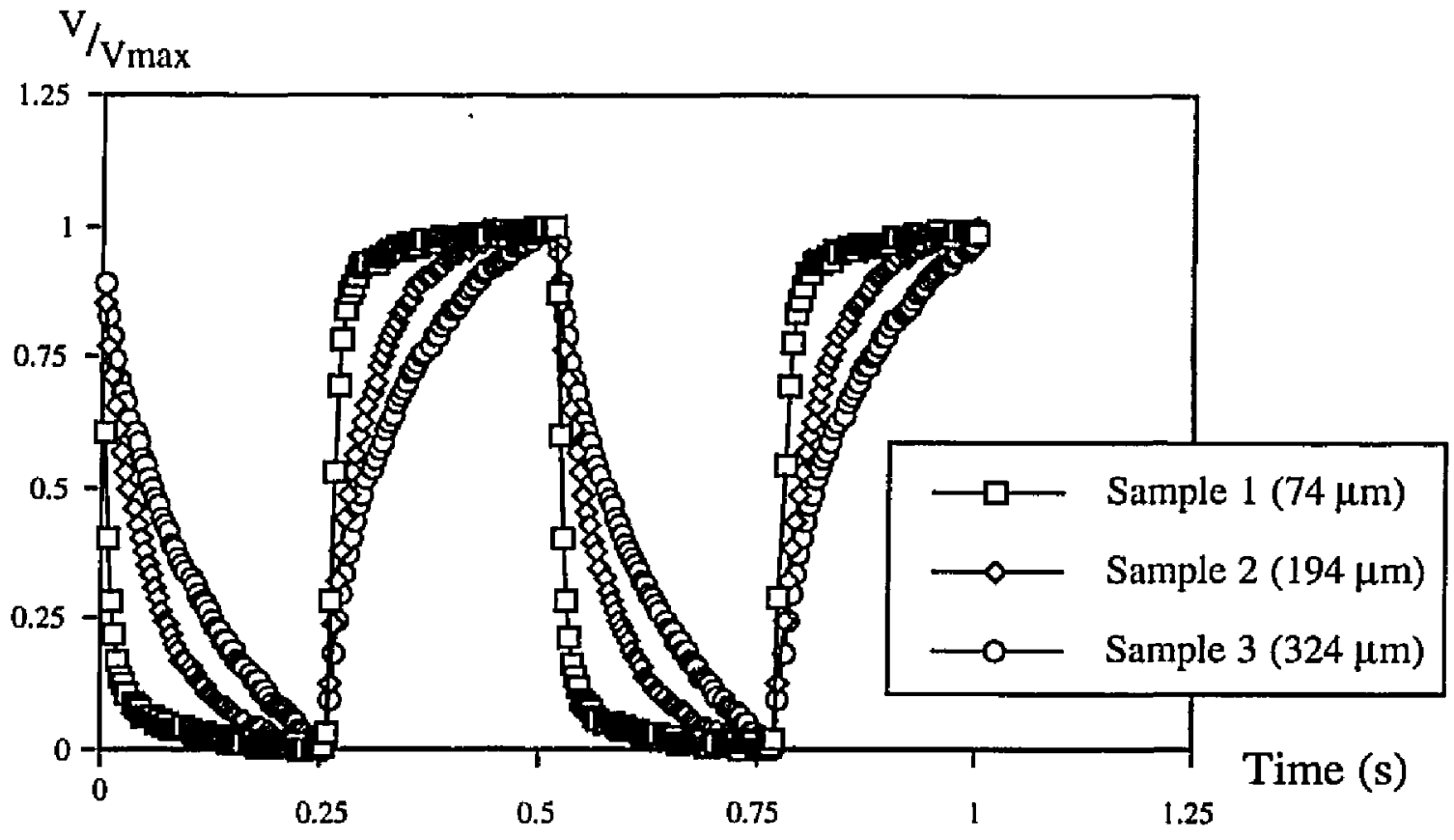

Fig. 4.2 Example of normalized temperature response of two layer specimens for an excitation frequency of $2 \mathrm{~Hz}$.

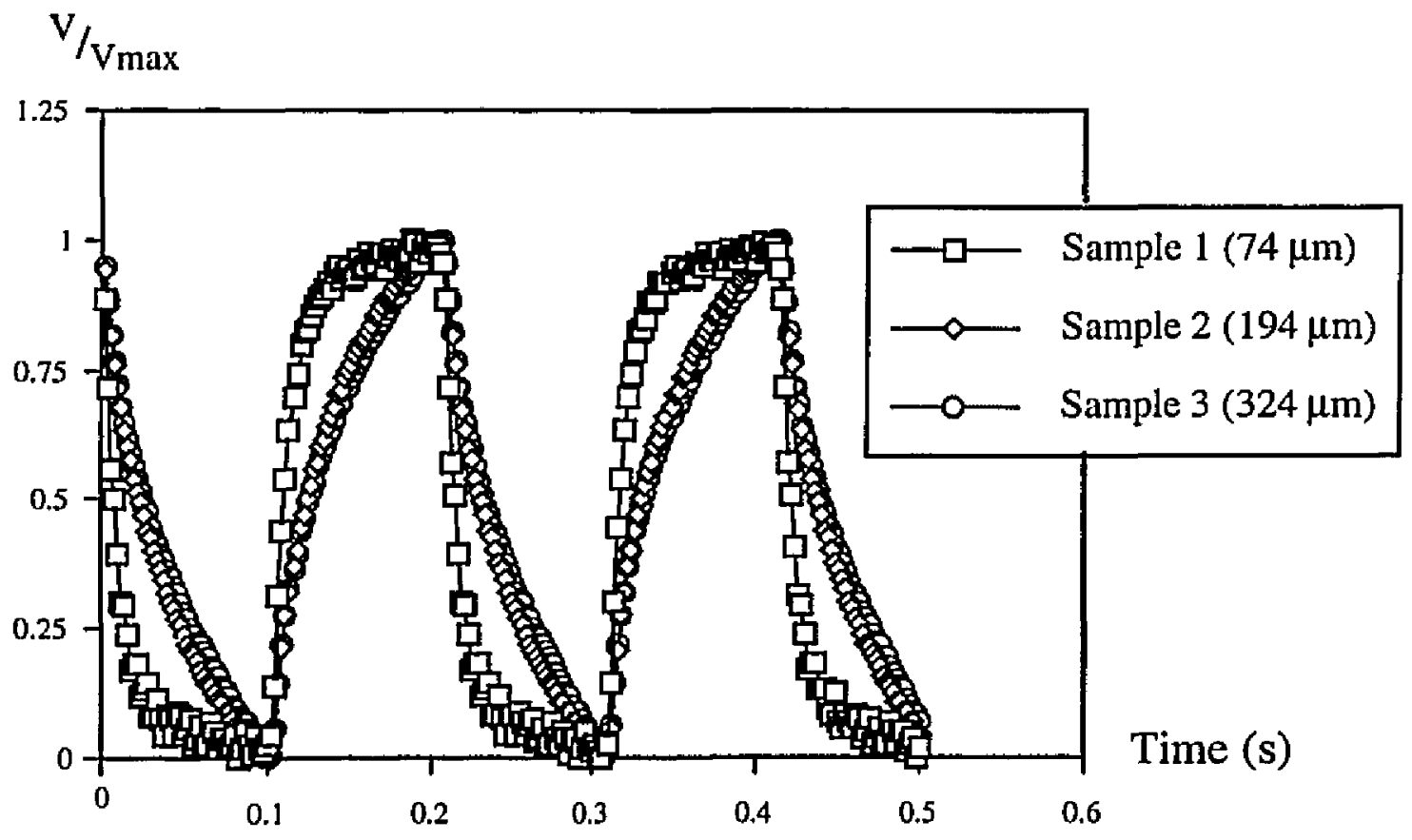

Fig. 4.3 Example of normalized temperature response of two layer specimens for an excitation frequency of $5 \mathrm{~Hz}$. 


\section{Thermal response}

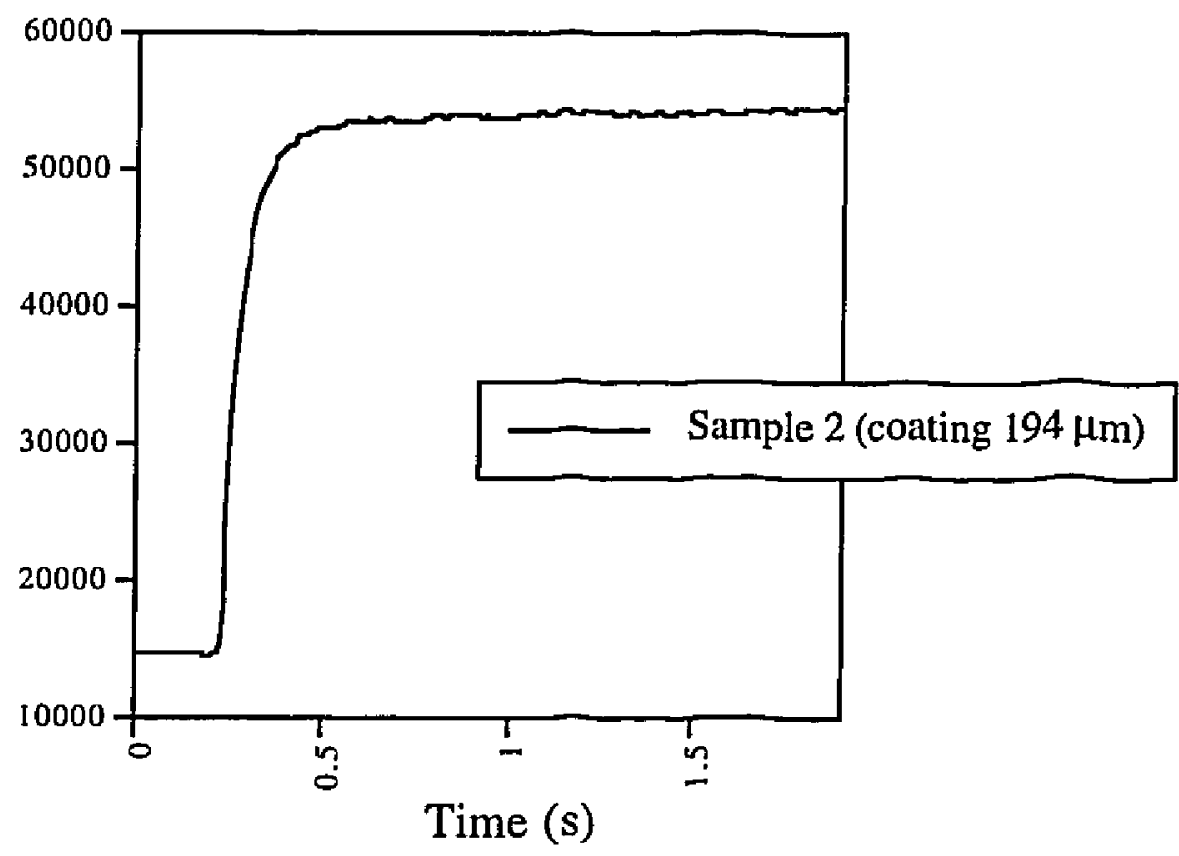

Fig. 4.4 Thermal response of sample 2 for an excitation frequency of $0.3 \mathrm{~Hz}$. Units for the vertical axis are arbitrary.

\section{Three layer samples}

The three layer samples examined in this section are described in chapter III. Through transmission thermal images using the scanned detector were obtained for all specimens except the one with bonded epoxy resin inner layer. While obtaining quantitative information is difficult from these images, they allow the detection of any irregularities in the specimens, such as voids or disbonds, prior to point measurements.

Results are first presented for a simulated corrosion sample. Then, data are shown for air and glycerin filled specimens. Finally, a typical time-temperature response for the epoxy inner layer specimen is shown. 


\section{a Simulated corrosion sample}

A detection technique for corrosion in multilayer structure has broad industrial applications. A typical need is the corrosion detection in lapjoints in airframe structures. Thermal analysis allows rapid inspection alternative to current methods such as visual inspection, ultrasonic or X-ray techniques. To investigate the applicability of this technique, a sample composed of outer aluminum layers with an inner $\mathrm{Al}(\mathrm{OH})_{3}$ layer is studied. This section presents typical data for this sample.

$\mathrm{Al}(\mathrm{OH})_{3}$ is a fine white powder that is easy to compact. Fabricating simulated corrosion samples requires a uniform distribution of the aluminum hydroxide between two aluminum plates. To assess the distribution after fabrication, a through transmission thermographic technique was used. A flash lamp provided an instantaneous planar heat deposit at the back surface. The temperature response at the front surface was measured with an infrared imager. $\mathrm{X}$-ray tomography images were also obtained for comparison with thermal images. Fig. 4.5 shows an example of contrast enhanced thermal image of the central region of the specimen. Also shown are X-ray tomography cross-sectional images at two locations on the sample. A comparison of the images enabled to map the corrosion product distribution in-between the plates. Point measurements of the thermal response following an impulse heat deposit at the opposite side were made at locations with known corrosion and air gaps. Typical data for these two regions are shown in Fig. 4.6. The front surface temperature increases at a slower rate in the case of regions with air gaps. As a result of the low volume specific heat of the air, heat transfers slower across the air gaps than in the regions with $\mathrm{Al}(\mathrm{OH})_{3}$. After approximately $8 \mathrm{~s}$, the front surface temperature reaches its maximum. It is the delay required for temperature to equalize across 
the sample.
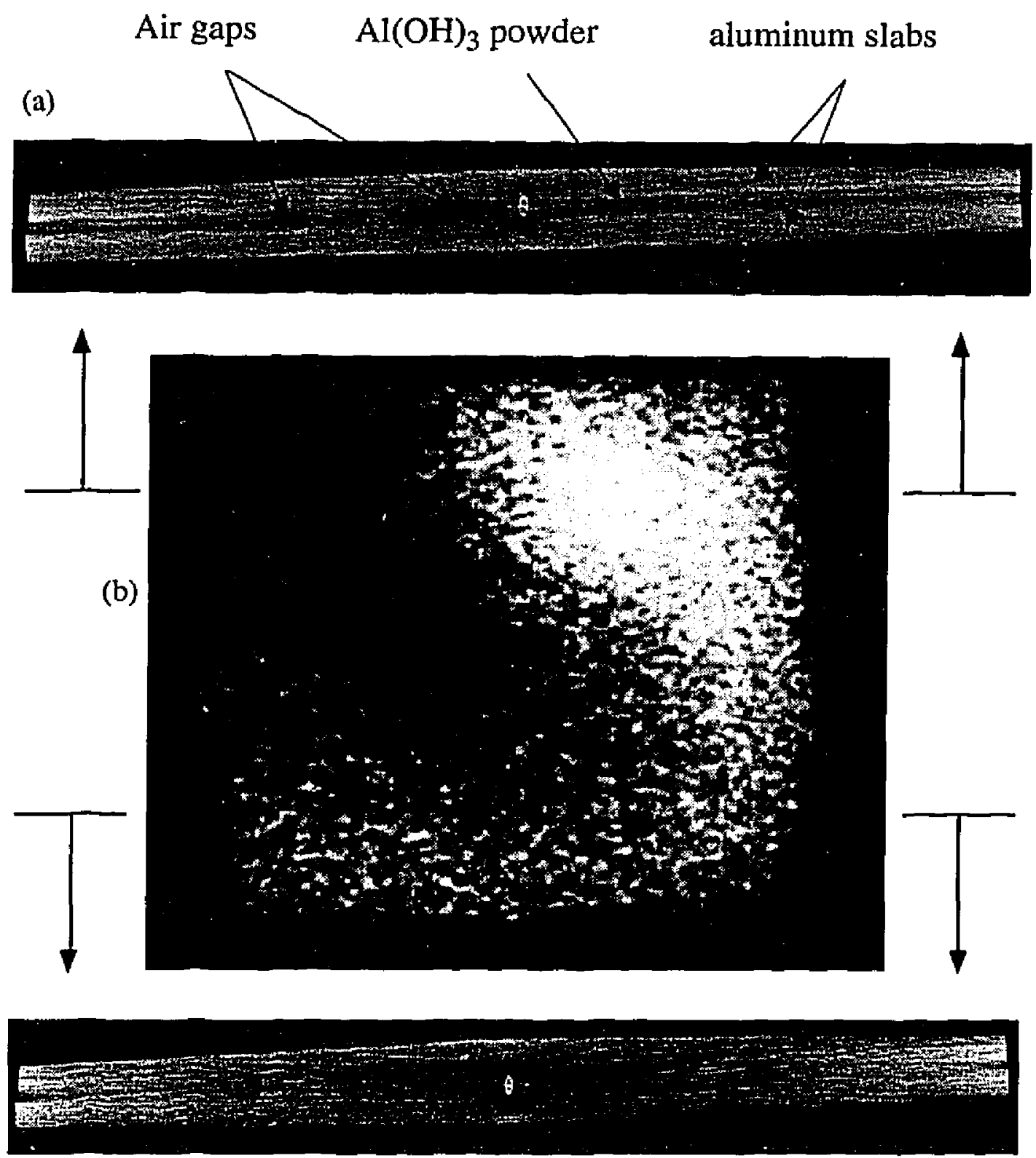

(c)

Fig. 4.5 X-ray tomography and thermal images of simulated corrosion specimen. Only the central region of the specimen is shown in the infrared image. (a) and (c) are the Xray tomography images. The ring shape in their center is a reconstruction artifact. 


\section{Thermal response}

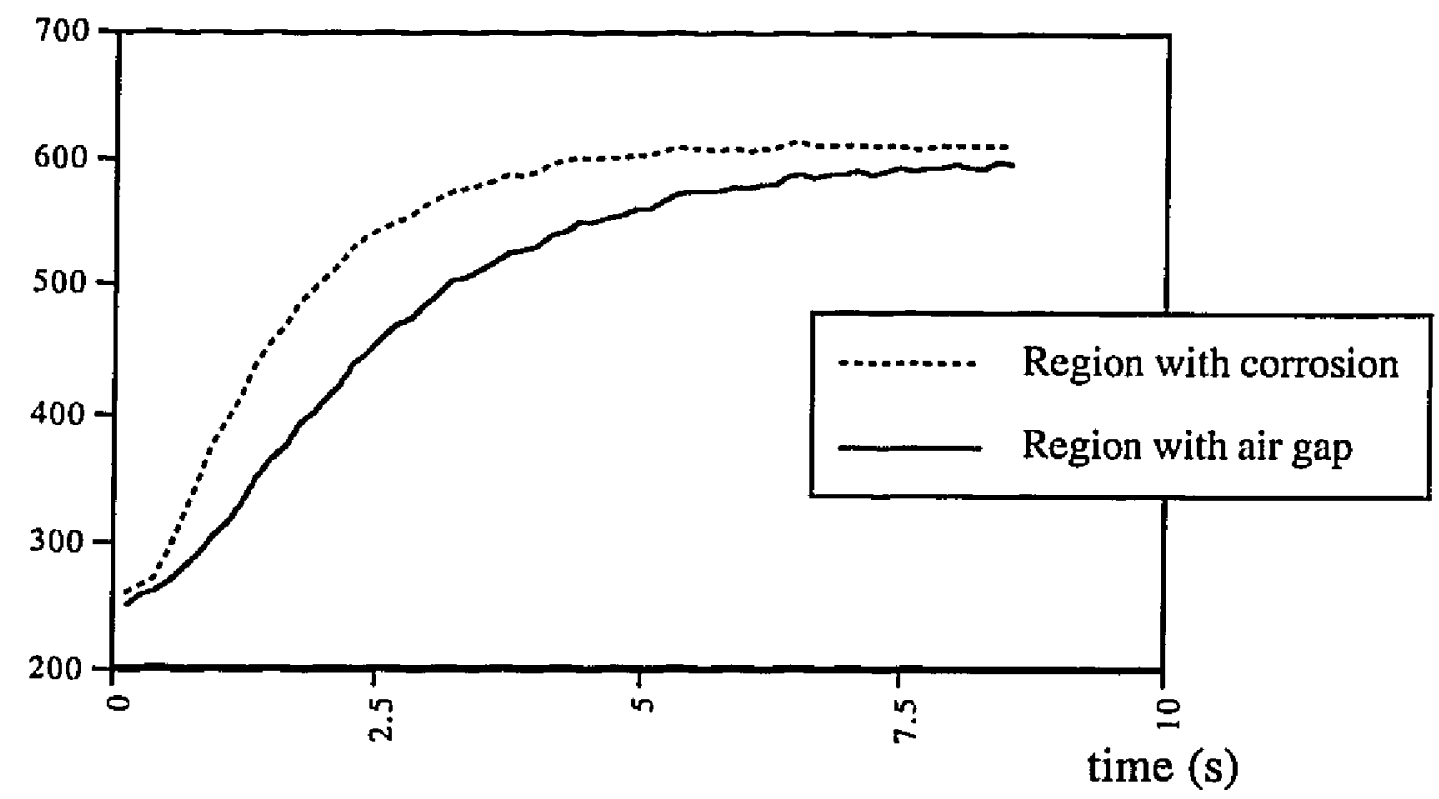

Fig. 4.6 Temperature profiles for simulated corrosion sample for two locations. To allow comparison, units for the vertical axis are the same for both curves. Heat is deposited at time $t=0$ at the back surface of the specimens.

From the temperature profiles shown in Fig. 4.6, it is possible to qualitatively differentiate regions with air gaps from regions with simulated corrosion. For structures with slow thermal response relative to video rate $(30 \mathrm{~Hz})$, these measurements can also be performed with a conventional imager. Subsequent processing of the data with techniques presented in the next chapter are required for a quantitative characterization.

\section{b Specimen with glycerin}

Fig. 4.7 shows a thermal image obtained with an infrared imager after a flash deposit at the back surface of the specimen. The image does not reveal any abnormal variations in thermal response. 


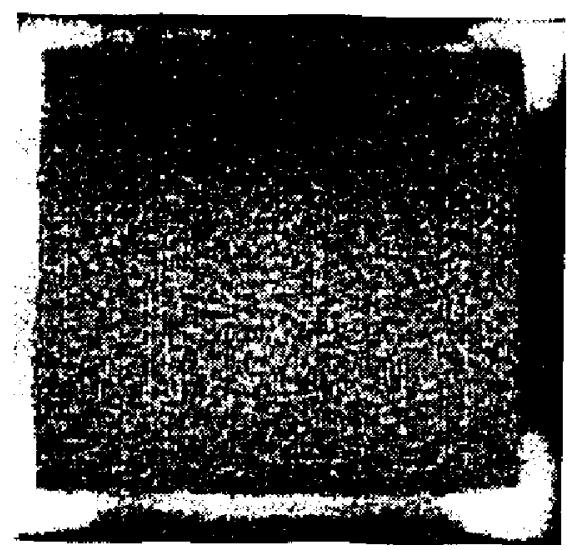

Fig. 4.7 Infrared image of the specimen with glycerin inner layer with flash heating at the back surface. The darker triangle in the upper right corner is a mark on the specimen surface made for orientation.

Two temperature profiles are shown in Fig. 4.8. The thermal responses follow an area heat impulse to the opposite side of the sample. Each of them is an average of ten measurements repeated at the same location on the specimen. They differ only in the time constant setting of the lock-in amplifier used to rectify the signal. The profile with a time constant of $1.25 \mathrm{~ms}$ has some visible noise. The time constant of the lock-in amplifier was chosen to sufficiently reduce the noise without significantly altering the shape of the response. 


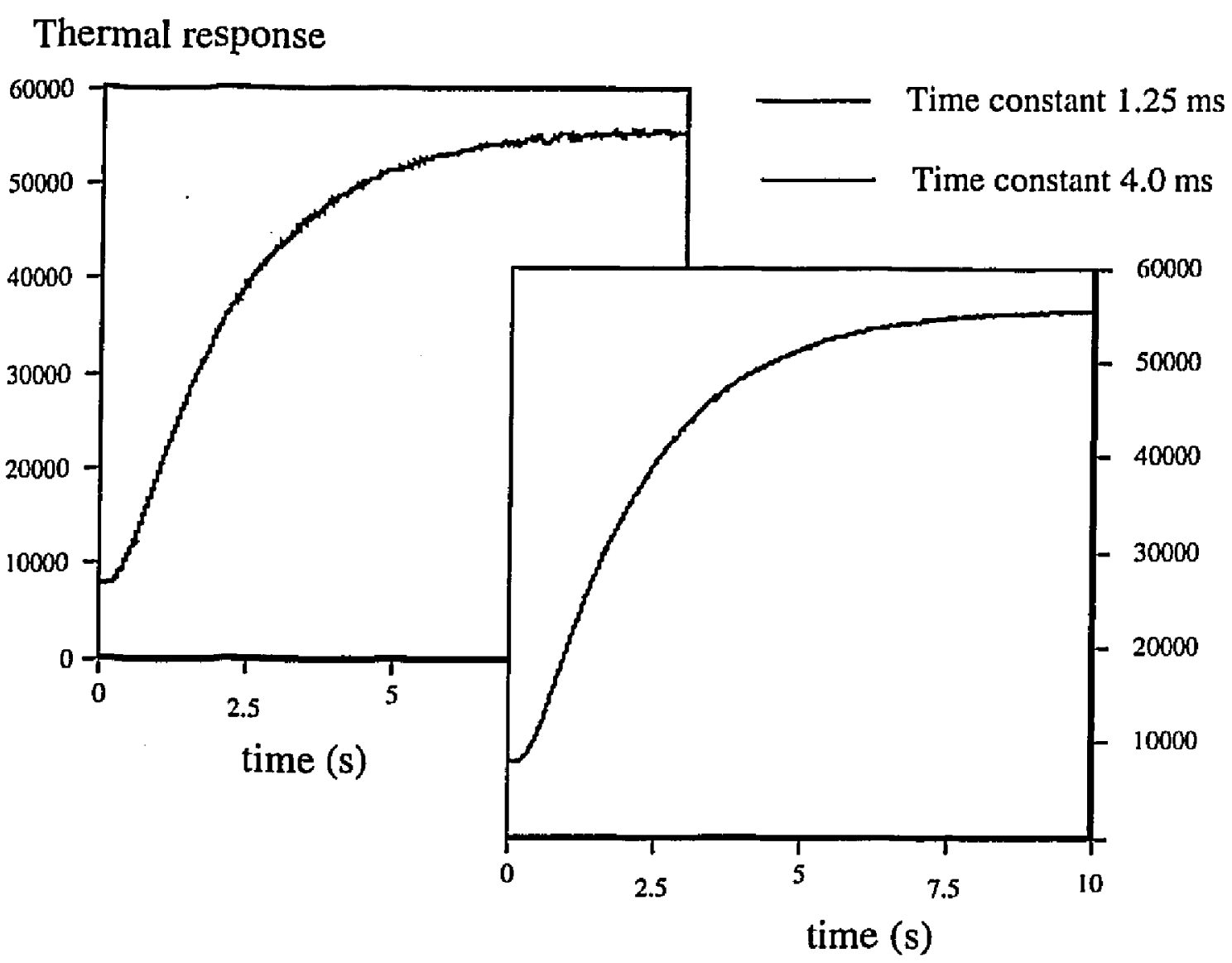

Fig. 4.8 Temperature profiles of two experiments for specimen with glycerin inner layer. Each profile is an average of ten data sets. Heat is deposited at time $t=0$. The time constant for the lock-in amplifier was set at 1.25 and $4.0 \mathrm{~ms}$. Units for the vertical axis are arbitrary.

\section{c Specimen with air}

Fig. 4.9 shows a thermal image of the three layer specimen with an inner layer of air. A flash lamp was used to deposit heat at the back surface of the sample. The most significant feature in the image is a variation from uneven heating. This variation can be removed by normalizing the temperature profiles at each point. Except for that variation, no anomalies appear in Fig. 4.9. The time-temperature profiles obtained for this structure are compara- 
ble to those obtained for the simulated corrosion specimen.

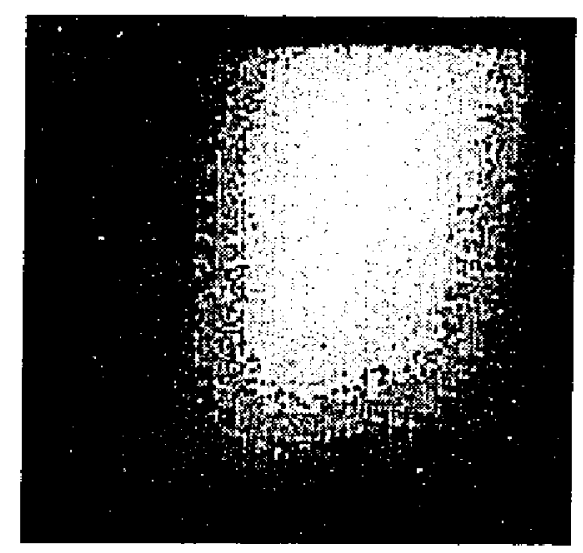

Fig. 4.9 Infrared image of the specimen with air inner layer with flash heating at the back surface.

\section{d Specimen with epoxy}

Bonded structures are increasingly used in industrial applications to replace riveted joints. The main advantage over riveted assemblies is an increase in performance to weight ratio, which makes them particularly interesting for aeronautic applications.

The data obtained for the specimens with an inner layer of epoxy resin exhibits the same characteristics as the samples with simulated corrosion, air gap or glycerin. After heat was deposited at the back surface, the front surface temperature starts rising with a time delay. Then, as heat equilibrates throughout the specimen, the rate of temperature change at the front surface approaches zero. The temperature of the whole specimen decreases slowly as convection losses cool the sample. To a good approximation, convection losses are proportional to the temperature gradient between the surface of a solid and the ambient atmosphere. Since all measurements were made at room temperature and the temperature elevation in the specimens was not significant, these convection losses were 
negligible during the measurement process. Typically, the measurement time is a few seconds while time for cool down is several minutes.

As seen on Fig. 4.10, maximum temperature is reached after approximately $4 \mathrm{~s}$, a time much shorter than other specimens. Several factors may contribute to these results. The epoxy layer is thinner than the inner layers in other specimens (i.e. $0.308 \mathrm{~mm}$ instead of $0.5 \mathrm{~mm}$ ). Moreover, epoxy is a relatively good thermal conductor. Finally, the resin is bonded to the aluminum slabs. The intimacy of the contact insures there is no thermal contact resistance at the interface between the aluminum and the adhesives.

\section{Thermal response}

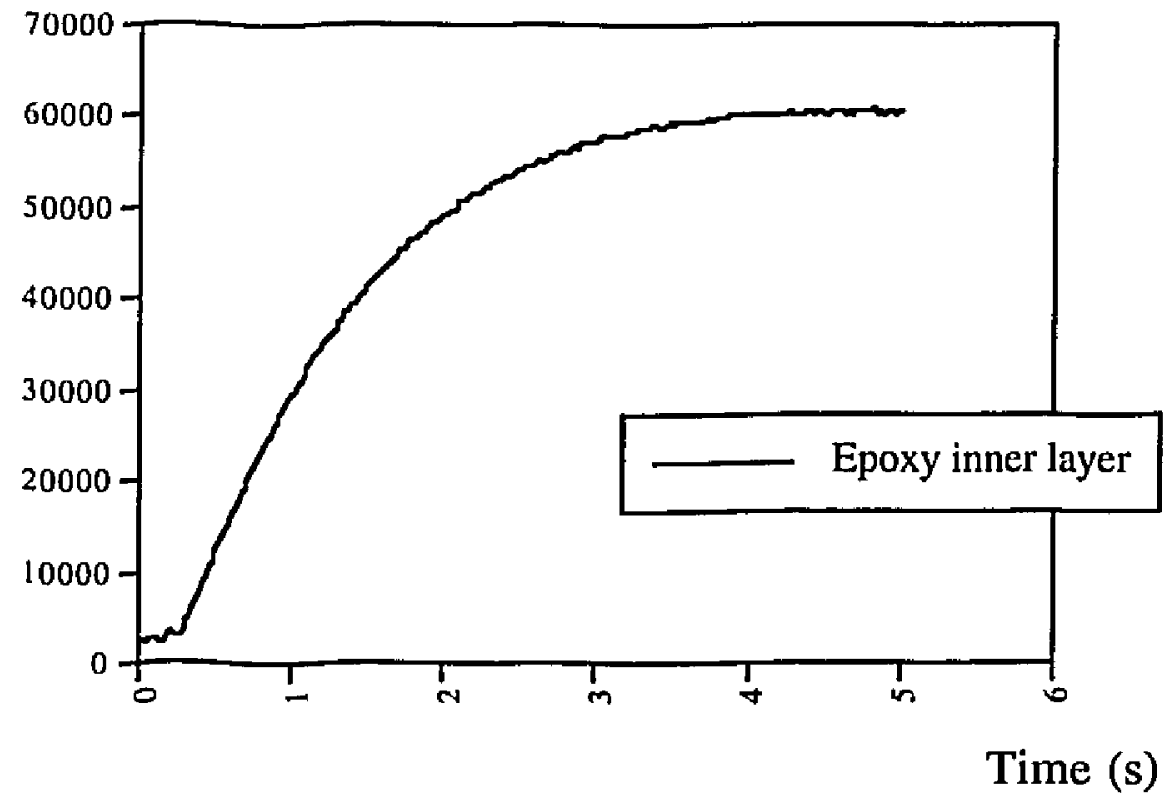

Fig. 4.10 Time-temperature profile for specimen with epoxy inner layer with flash deposit on the back surface. Instantaneous heat deposit is made at $t=0$. Units for the vertical axis are arbitrary. 


\section{B Temperature profiles and images for composite materials}

\section{Reaction Bonded Silicon Nitride / SiC fiber samples}

Reaction Bonded Silicon Nitride composite materials with SiC fibers are good candidates for high temperature applications in hostile environments. Nondestructive evaluation techniques of such materials is an active area of research $[15,16,18,19,20,21]$. Prior measurements have mainly focused on the effective or bulk thermal properties of such composite materials. A point detection technique enables obtaining time-temperature profiles with a high enough time and spatial resolution to resolve individual fibers. Two fiber reinforced composite specimens described in chapter III were studied. The fibers have a diameter of $150 \mu \mathrm{m}$ and they are separated by $100 \mu \mathrm{m}$. Specimen RBSN 1 is as originally fabricated and RBSN2 was degraded by heat treatment to induce damage.

The measurement of the thermal response at each point gives a large amount of information which is difficult to visualize in its raw form. For improved visualization, it is suitable to reduce the data to a form where each data point is represented by a limited number of parameters. A convenient reduction is the Fourier transform of the time domain record. The response at each point is represented by its Fourier component.

The higher frequency terms in the Fourier transform correspond to the response nearer to the surface of the composite. Therefore, with proper selection of the excitation frequency, only the first few harmonics will contain information on the fibers. Consequently, only these first few terms need to be displayed. The processing of the data is presented graphically in Fig. 4.11. 
Temperature

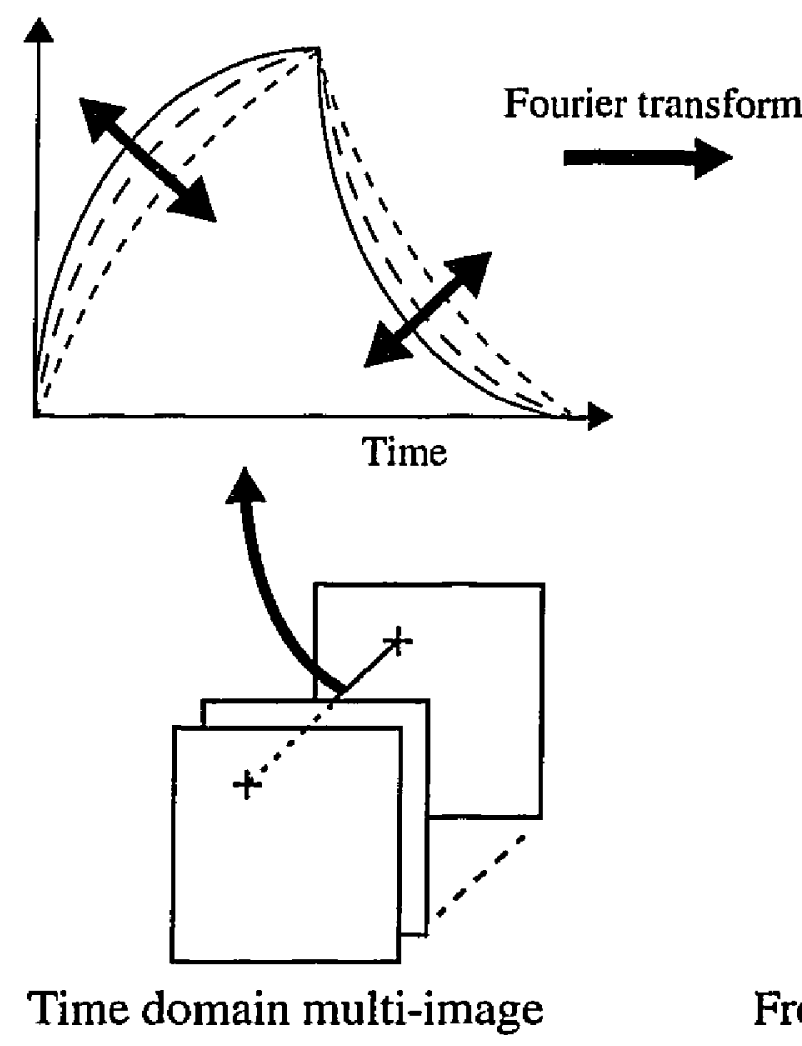

Amplitude
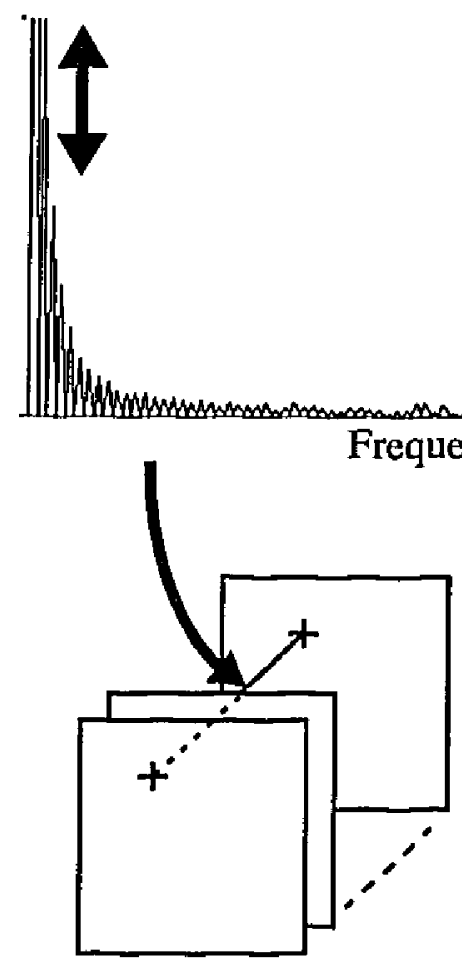

Frequency domain multi-image

Fig. 4.11 Pixel by pixel Fourier transform for a multi-image. The Fourier transform of the temperature profiles at each pixel in the time domain multi-image is taken to create a frequency domain multi-image.

A contrast enhanced time domain surface temperature image at a fixed time in the heating cycle of specimen RBSN1 can be seen in Fig. 4.12. Surface defects are indicated. A repeated arrangement of vertical shapes is also visible. Their spacing corresponds to the spacing of the embedded SiC fibers but the pattern is not clear. The first Fourier harmonic image, also shown in Fig. 4.12, clearly displays the fiber layout as well as the surface defects. The first frequency component image corresponds to the temperature response at the deepest parts of the specimen reached by a significant amount of heat. As shown in Fig. 4.13, for the excitation frequency chosen, the first harmonic image contains most of 
the signal due to the fibers.

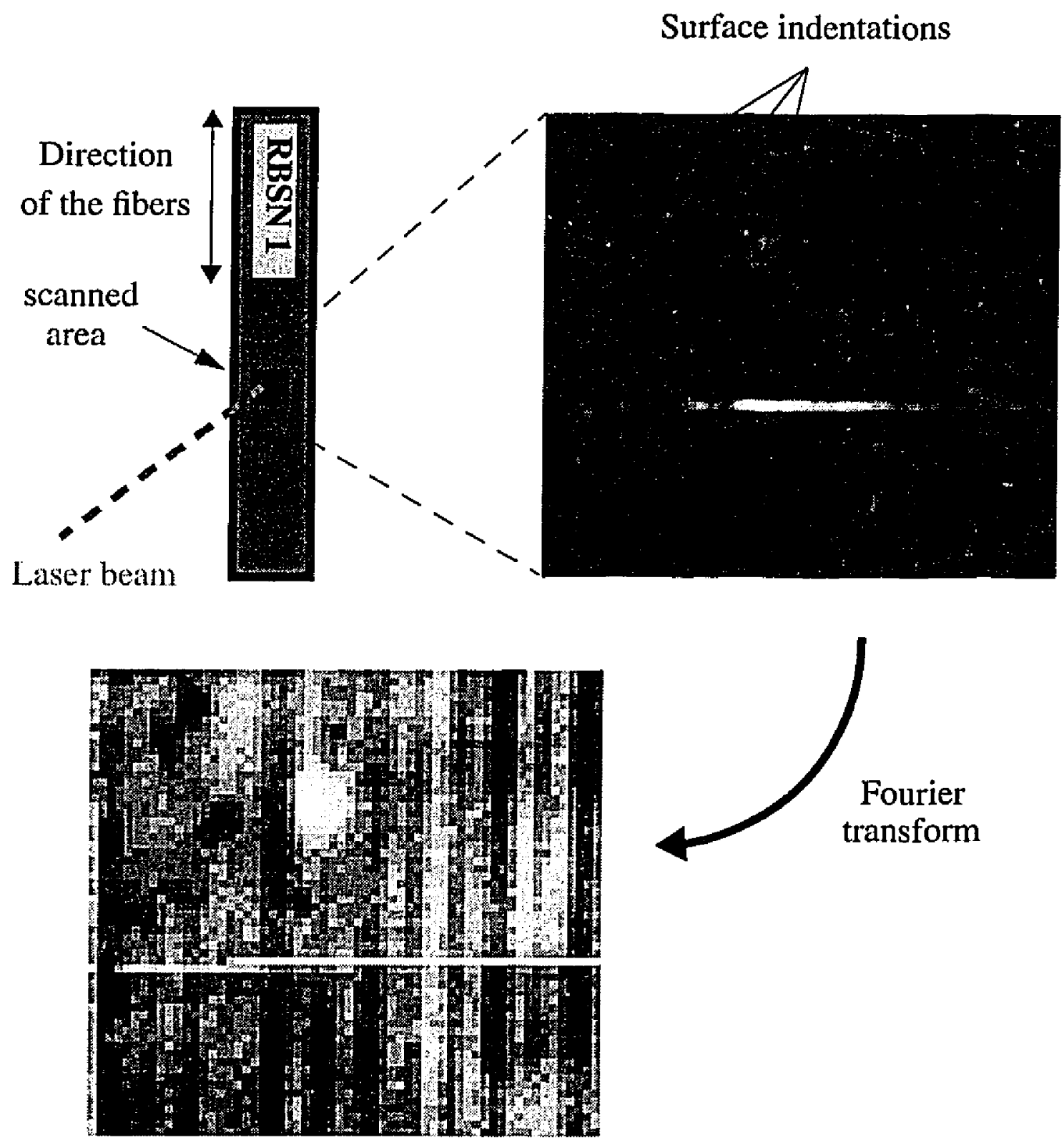

Fig. 4.12 Example of time and frequency domain contrast enhanced images of the temperature response of original sample RBSN1. The scanned area is a square of $3 \mathrm{~mm} \mathrm{x}$ $3 \mathrm{~mm}$. The image is $60 \times 60$ pixels. Laser power was set for $1.0 \mathrm{~W}$ and the beam was chopped by an acousto-optic modulator at $50 \mathrm{~Hz}$. The horizontal line on the images is a camera artifact due to $\mathrm{LN}_{2}$ refilling of the infrared detector dewar. 
The first four odd harmonics of the frequency domain multi-image are displayed. The higher frequency components, shown in Fig. 4.13 (c) and (d), are dominated by noise. The surface defects, responsible for the largest variations, are visible on the first three images. The higher harmonics component images, not represented in Fig. 4.13, do not bring any useful information.

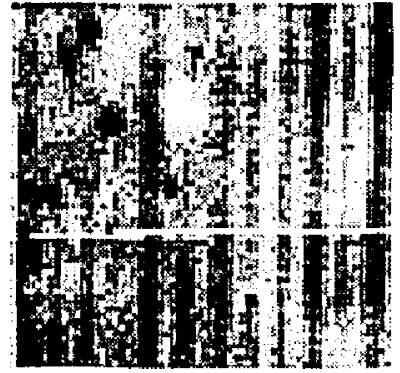

(a)

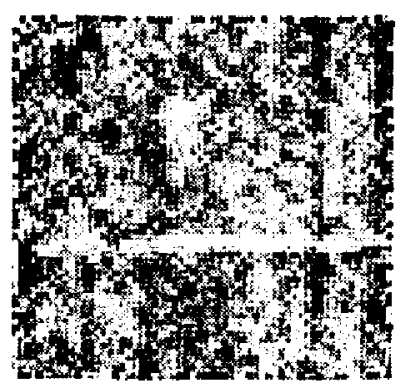

(c)

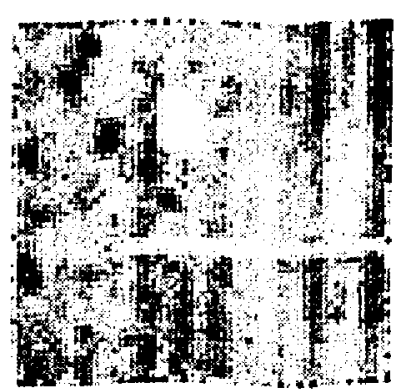

(b)

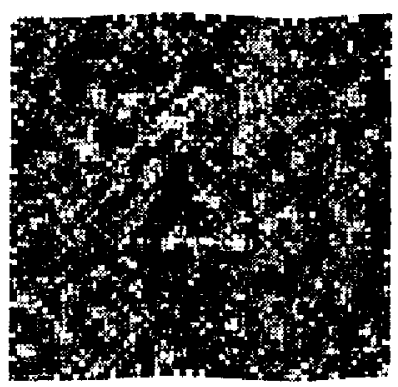

(d)

Fig. 4.13 First Harmonics of the frequency domain multi-image for RBSN1. (a), (b), (c) and (d) are respectively the first, third, fifth and seventh harmonics.

A similar study of the heat damaged specimen was conducted. The results are presented in Fig. 4.14 and 4.15. The time domain images are not shown. Although visual inspection did not reveal any surface damage, the Fourier harmonic images shows significant variations in thermal response. Subsurface changes in thermal properties within the 
scanned area are responsible for these variations. The dimensions of the scanned area and the spatial resolution of the images for both RBSN1 and RBSN2 are the same.

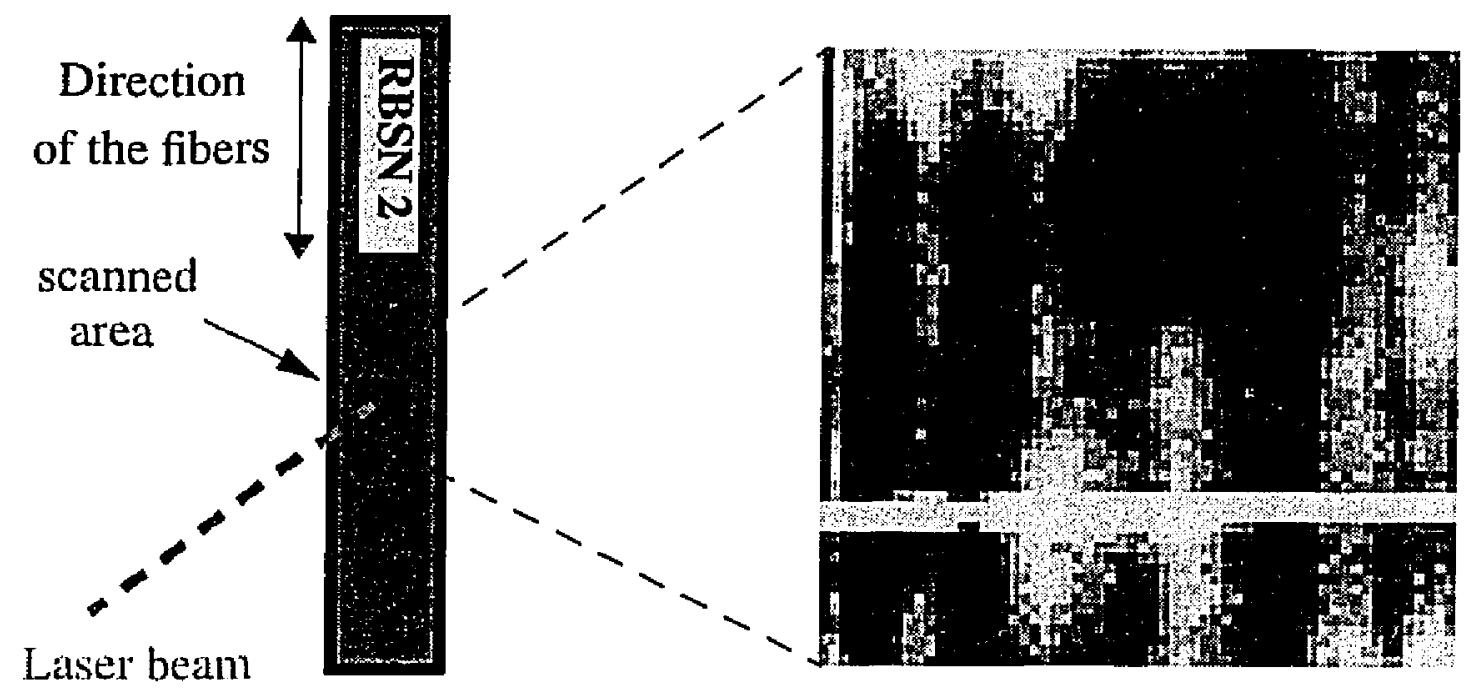

Fig. 4.14 Frequency domain image of the temperature response of degraded sample RBSN2. The scanned area is a square of $3 \mathrm{~mm} \times 3 \mathrm{~mm}$. The image is $60 \times 60$ pixels. Laser power was set for $1.4 \mathrm{~W}$ and the beam was chopped by an acousto-optic modulator at $50 \mathrm{~Hz}$. The horizontal line in the lower half of the image is an artifact due to $\mathrm{LN}_{2}$ refilling of the infrared detector dewar.

In Fig. 4.15 (a), (b) and (c), individual fibers are visible. Larger regions in dark also appear. They cover extended areas, spreading across the fibers. Some vertical patterns are still visible in Fig. 4.15 (d) but the noise level is higher than in the first three odd frequency domain images. The image (a) reveals extended damage but (b) and (c) show more details about the structure of specimen RBSN2. 


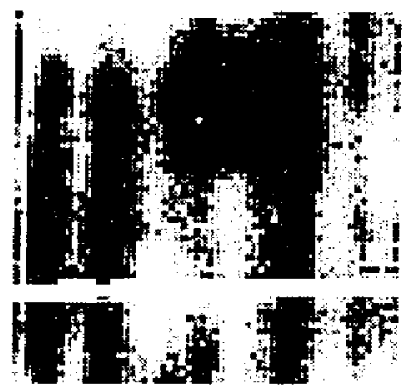

(a)

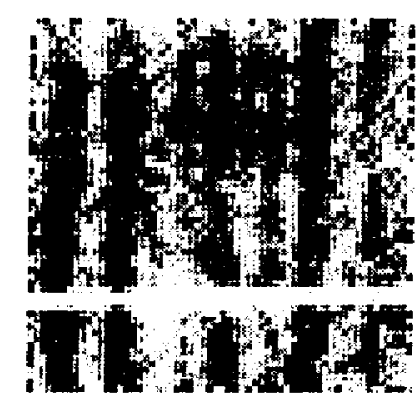

(c)

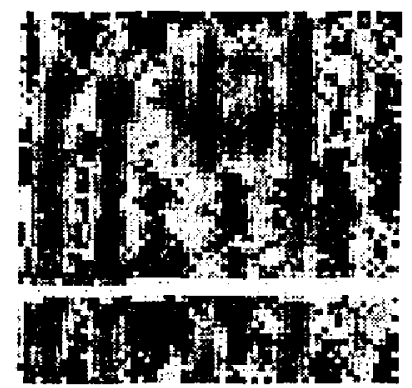

(b)

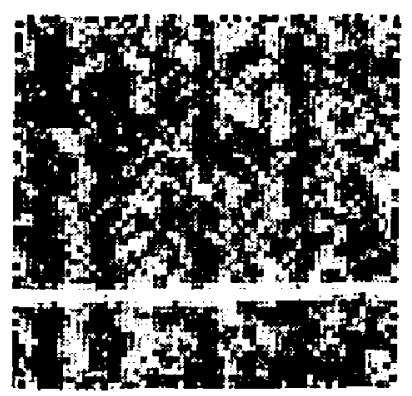

(d)

Fig. 4.15 First Harmonics of the frequency domain multi-image for RBSN2. (a), (b), (c) and (d) are respectively the first, third, ffth and seventh harmonics.

\section{Aluminum fabricated specimen}

Fig. 4.16 shows the first harmonic image for the aluminum specimen described in chapter III. A vertical pattern that corresponds to the presence of the hole is visible.

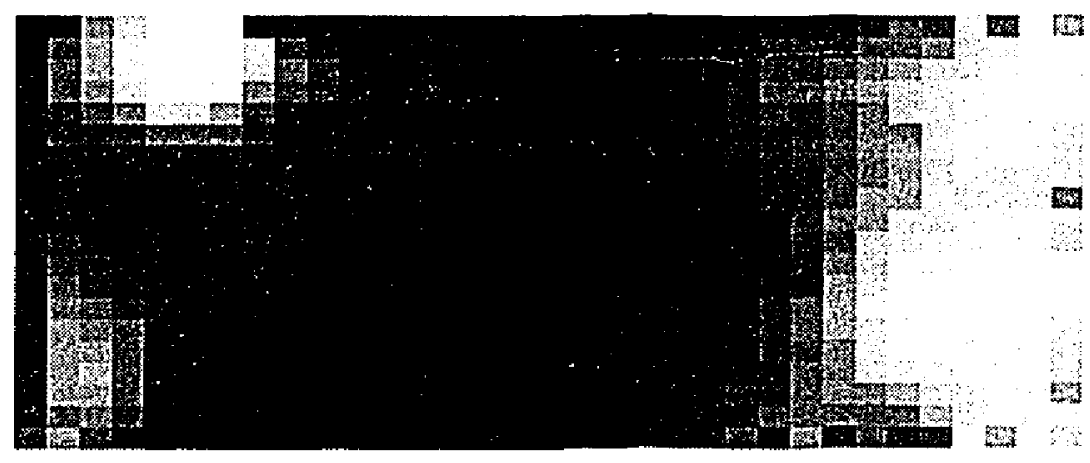

Fig. 4.16 First harmonic frequency domain image of aluminum specimen. 


\section{CHAPTER V}

\section{QUANTITATIVE ANALYSIS AND INTERPRETATION}

Last chapter presented experimental data obtained with different setup configurations described in chapter III. Little or no processing was used. Temperature profiles for two and three layered specimens were simply displayed as normalized graphs. Data obtained on composite materials was displayed as images in time and frequency. Presented in this form, only qualitative information could be obtained. Further analysis is needed to characterize the specimens from a quantitative point of view.

This chapter begins with a discussion of the nonlinear least square fitting routine that was used to obtain the thermal properties of multilayered materials. The algorithm used in all cases allowed a multiparameter fit of the experimental data by the temperature response models developed in chapter II. The last part of this chapter presents results for flat and fiber reinforced composite structures.

\section{A Flat geometry, thermal properties}

\section{Nonlinear least square fitting}

The least square fit routine used in this work is a standard one: a merit function $\chi^{2}$ is 
defined and its minimization, an iterative process, determines the best fit parameters. The minimization is stopped when $\chi^{2}$ stops decreasing. The iterative process follows Levenberg-Marquardt method. When the trial values are far from the solution, the method of steepest descent is used. When they approach the minimum, the $\chi^{2}$ function is approximated by a quadratic form and the minimum trial parameters are estimated accordingly.

\section{Results for two layer samples}

Data was processed to obtain quantitative information for the three samples introduced in chapter III. For each experiment, the two layer model presented in chapter II was fitted to the data and the thermal diffusivity and conductivity of the coating were calculated.

Fig. 5.1 and 5.2 show the results obtained. For samples 1 and 2, the values are independent from the excitation frequency. For sample 3, as the frequency increases, the estimated values diverge. This is a result of the thermal transient times of the coating becoming significant relative to the period of the thermal excitation. Under such conditions, the thermal response is a result of the paint alone and the two layer model is no longer required. 


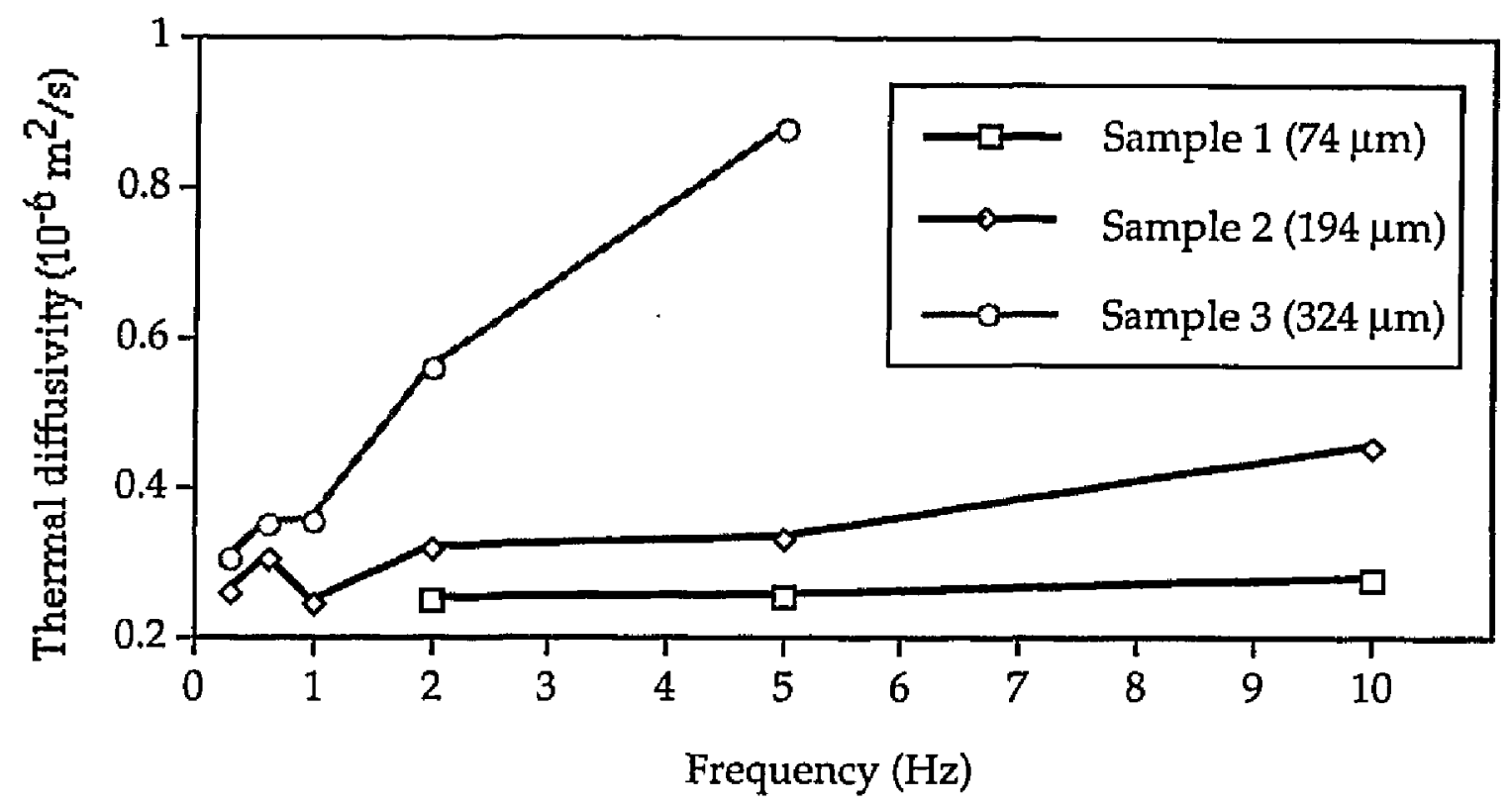

Fig. 5.1 Estimated thermal diffusivity of coating as a function of frequency for two layer samples with varying coating thickness.

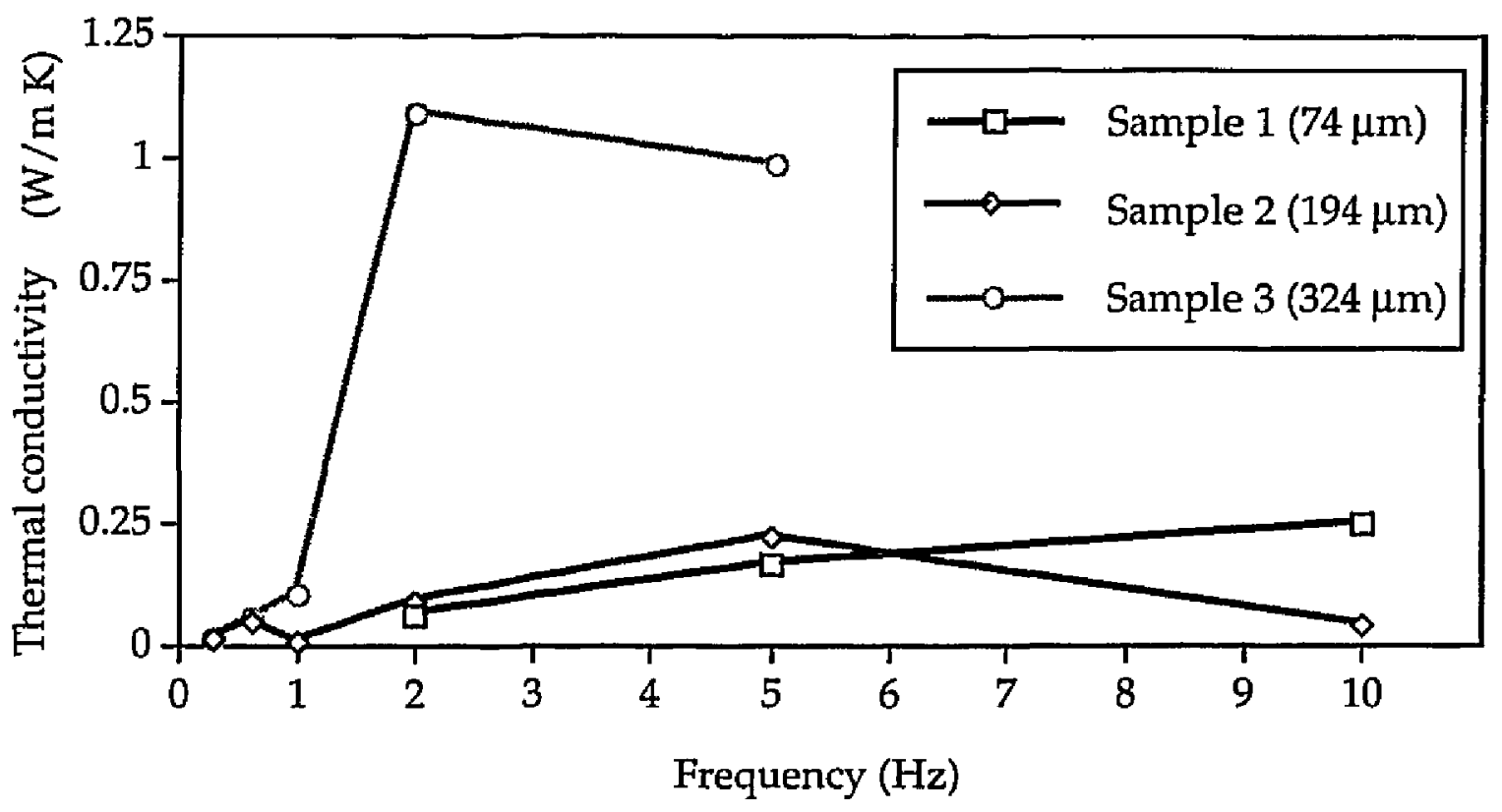

Fig. 5.2 Estimated thermal conductivity of coating as a function of frequency for two layer samples with varying coating thickness. 
Computer simulations shown in Fig. 5.3 of the temperature profiles in the first layer at different times after a step heating starting at $t=0$ verify this conclusion. The thermal properties used in the simulations are the experimentally determined values of Fig. 5.1 and 5.2. Fig. 5.3 (a), (b) and (c) refer to, respectively, samples 1, 2 and 3. The profiles in Fig. 5.3 (a) and (b) exhibit a linear drop across the first layer. The $0.1 \mathrm{~s}$ line in Fig. 5.3 (b) shows a small non linear component. For Fig. 5.3 (c), the $0.1 \mathrm{~s}$ line is the result of a strong non linear component: the temperature gradient throughout the first layer for times under 1 second is dominated by the thermal response of the paint. From thermal responses for excitation frequencies above $1 \mathrm{~Hz}$, the thermal properties of the second layer cannot be determined. For lower frequencies, the transient thermal responses become less significant and standard methods can be used to characterize the second layer.

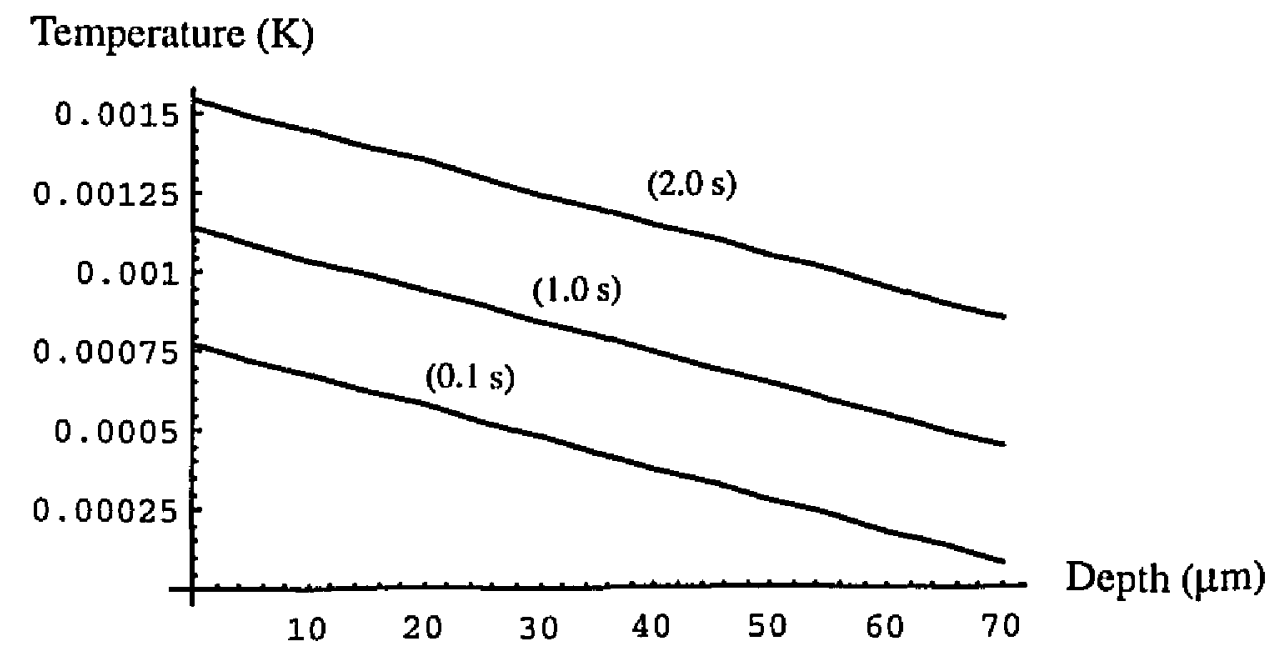

Fig. 5.3 (a) Simulations of temperature profiles in the first layer of two layer sample 1 after $0.1,1.0$ and 2.0 seconds. Temperatures are in Kelvin per unit flux, depths are in $\mu \mathrm{m} .0 \mu \mathrm{m}$ is at the surface. 
Temperature (K)

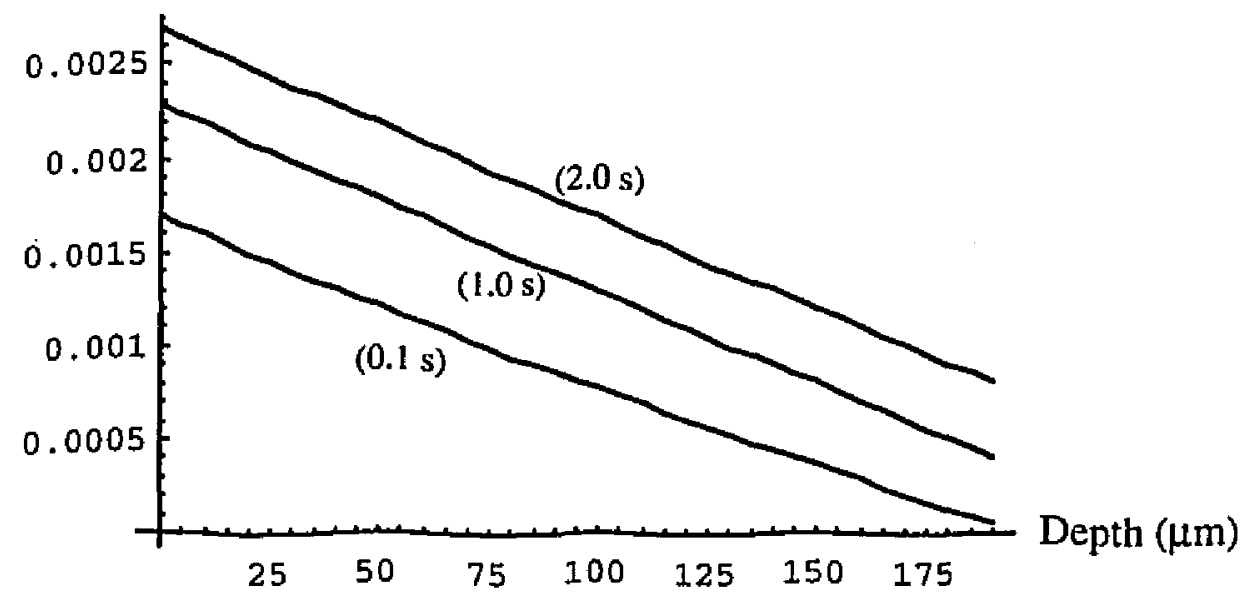

Fig. 5.3 (b) Simulations of temperature profiles in the first layer of two layer sample 2 after $0.1,1.0$ and 2.0 seconds.

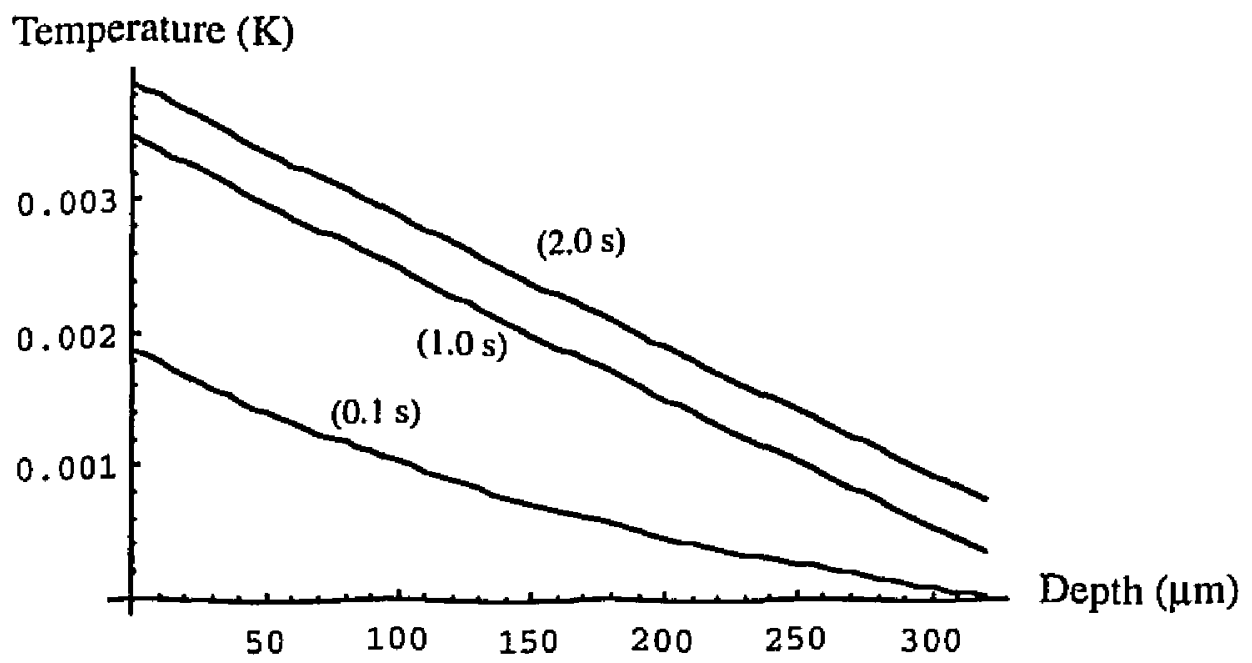

Fig. 5.3 (c) Simulations of temperature profiles in the first layer of two layer sample 3 after $0.1,1.0$ and 2.0 seconds.

\section{Results for three layer samples}

Table 5.1 shows a summary of the results obtained from non linear least square fits.

The samples used were presented in chapter III. The inner layers were composed of, 
respectively: $0.52 \mathrm{~mm}$ of air, $0.52 \mathrm{~mm}$ of glycerin, $0.308 \mathrm{~mm}$ of epoxy resin and $0.5 \mathrm{~mm}$ of $\mathrm{Al}(\mathrm{OH})_{3}$. The results are generally in good agreement with other literature values except for the measured conductivity of air that appears too high. This poor result may be explained by the heat transfer due to the convection in the interior gap that is not taken into account by the model.

\begin{tabular}{|l|r|r|r|r|}
\hline & \multicolumn{2}{|c|}{ diffusivity $\left(10^{-6} \mathrm{~m}^{2} / \mathrm{s}\right)$} & \multicolumn{2}{c|}{ conductivity (W/m K) } \\
\hline & measured & literature value & measured & literature value \\
\hline \hline Air & 0.22 & $0.23[27]$ & 0.05 & $0.026[30]$ \\
\hline Glycerin & 0.084 & $0.094[28]$ & 0.39 & $0.30[31]$ \\
\hline Epoxy resin & 0.05 & $0.1-0.3[29]$ & 0.41 & $0.16-0.4[29]$ \\
\hline $\begin{array}{l}\text { Simulated } \\
\text { Corrosion }\end{array}$ & 0.063 & not available & 0.69 & 0.675 \\
\hline
\end{tabular}

Table 5.1 Measurements of thermal diffusivity and conductivity and comparison with literature values for three layer specimens.

\section{B Composite materials, thermal properties}

\section{Fabricated aluminum specimen}

Fig. 5.4 shows the column average values for the scanned area on the specimen. The values for each column in the frequency response images were averaged to improve the signal. Superimposed is a simulation for the thermal properties of aluminum and $\mathrm{K}_{\mathrm{fiber}}=$ 0 . A reasonably good agreement is obtained between the simulation and the experimental data. The differences between the simulated profile and the actual data are possibly due to the in-plane diffusion effects. 
Average column value

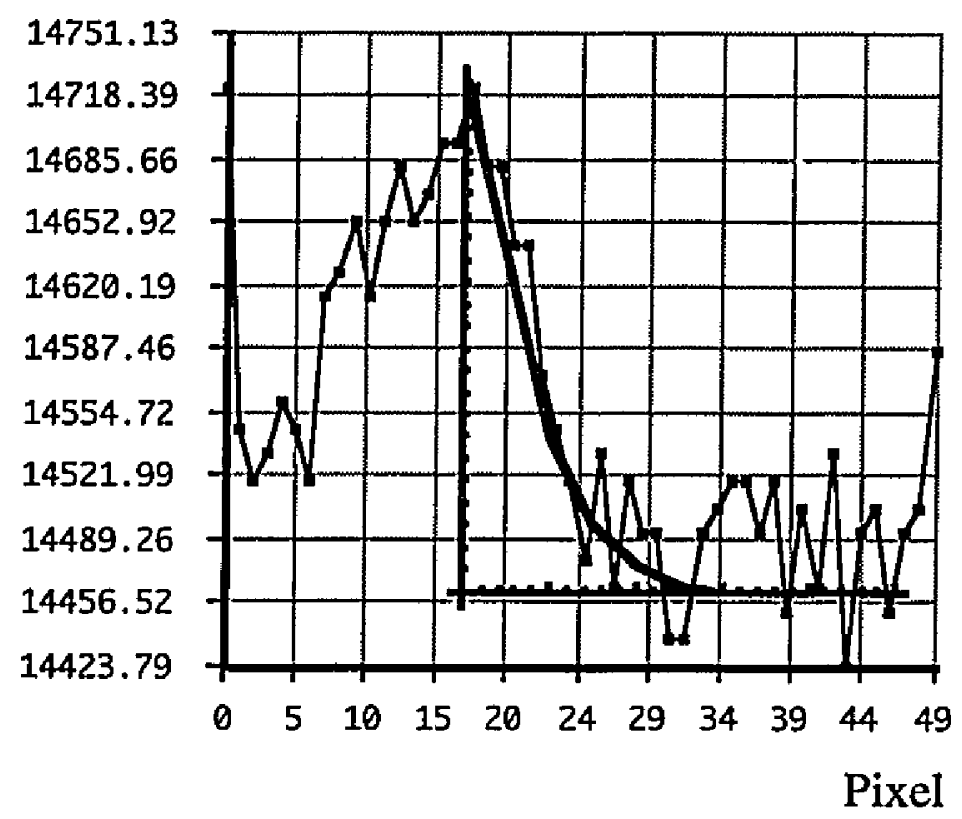

Fig. 5.4 Column average values of the third harmonic of the Fourier transform of the surface temperature for fabricated aluminum specimen with air hole. The excitation frequency is $20 \mathrm{~Hz}$. A profile obtained from a simulation is also represented. It was obtained for thermal properties: $\alpha_{1}=5010^{-6} \mathrm{~m}^{2} / \mathrm{s}$ and $\mathrm{K}_{2}=120 \mathrm{~W} / \mathrm{m} \mathrm{K}$.

\section{Reaction Bonded Silicon Nitride specimens}

The same averaging method used for the images of the fabricated aluminum specimen was applied to the results obtained for the two RBSN composite samples. Results for RBSN1 are shown in Fig. 5.5 (a), (b), (c) and (d) and for RBSN2 in Fig. 5.6 (a), (b), (c) and (d).

In this case, performing a fit is not appropriate. As stated in chapter II, the model is only valid if the spacing between the fibers is large compared to $\sqrt{\frac{\alpha}{\omega}}$. For an estimated room temperature matrix thermal diffusivity $\alpha=310^{-6} \mathrm{~m}^{2} / \mathrm{s}$ in a direction perpendicular 
to the fibers [20] and an excitation frequency of $50 \mathrm{~Hz}$, one wavelength is approximately $0.86 \mathrm{~mm}$. The depth of penetration of the thermal signal is slightly inferior but includes several fiber layers. The interactions between them cannot be neglected. However, in the case of specimen RBSN1, the thermal response is strongly dominated by the first layer. This is shown in Fig. 5.5 (a) where the thermal response of the fibers corresponds to the dips in the average pixel value profiles. This is due to the higher thermal properties of the fibers which act as heat sinks in the composite structure. The profiles represented in Fig. 5.5 (b), (c) and (d) lead to the same conclusion. The noise level increases as higher harmonics are considered.

After damages are thermally induced, the effective bulk properties of the composite structure are affected but the thermal conductivity/diffusivity of the matrix are not dramatically decreased. As a consequence, it is expected that the depth of penetration of the thermal signal is approximately the same in the case of the degraded specimen RBSN2. The column average values for the first odd harmonic image shown in Fig. 5.6 (a) reveals extended thermally affected areas. This response is due to the inner structure damages located deeper than the first fiber layer. Moreover, the average column profile in Fig. 5.6 (a) is much smoother than any other represented in Fig. 5.5 and 5.6, supporting the conclusion that the response is due to deep subsurface defects. The higher harmonic average column profiles shown in Fig. 5.6 (b), (c) and (d) are less significantly affected by the thermally induced damages because the depth of penetration of the thermal signal is reduced. The thermal response due to the first fiber layer is clearly visible on these images. 


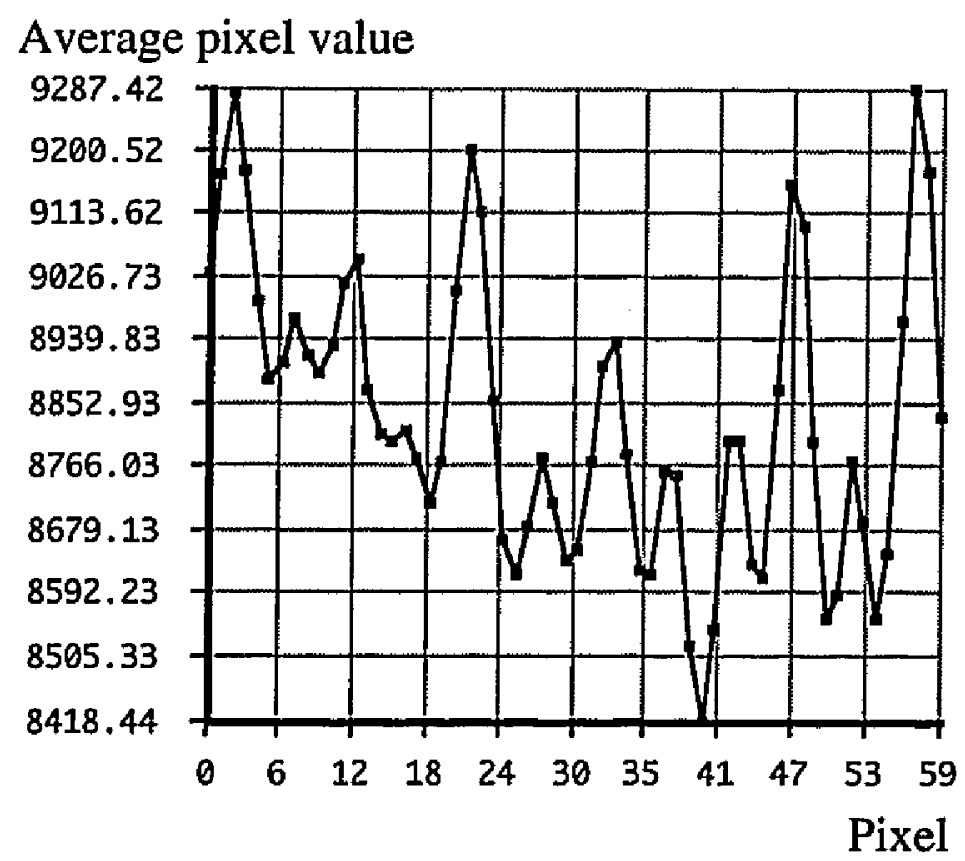

Fig. 5.5 (a) Column average for the first harmonic frequency domain image of specimen RBSN1.

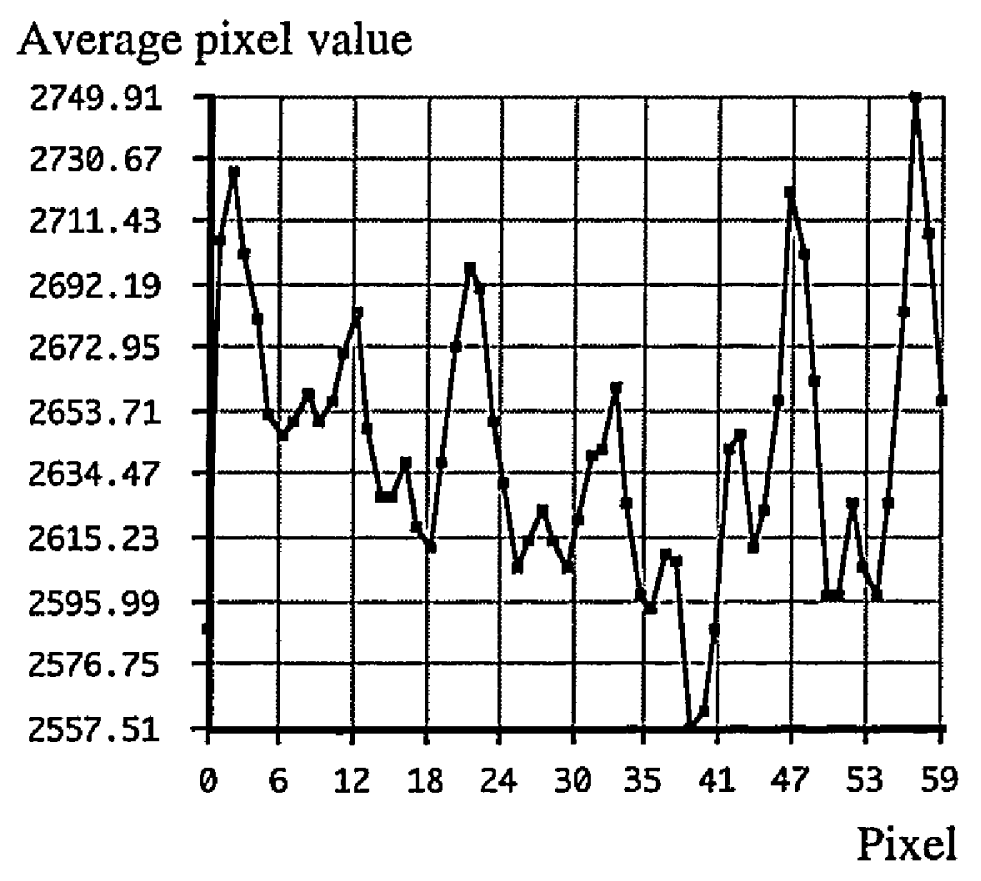

Fig. 5.5 (b) Column average for the third harmonic frequency domain image of specimen RBSN1. 
Average pixel value

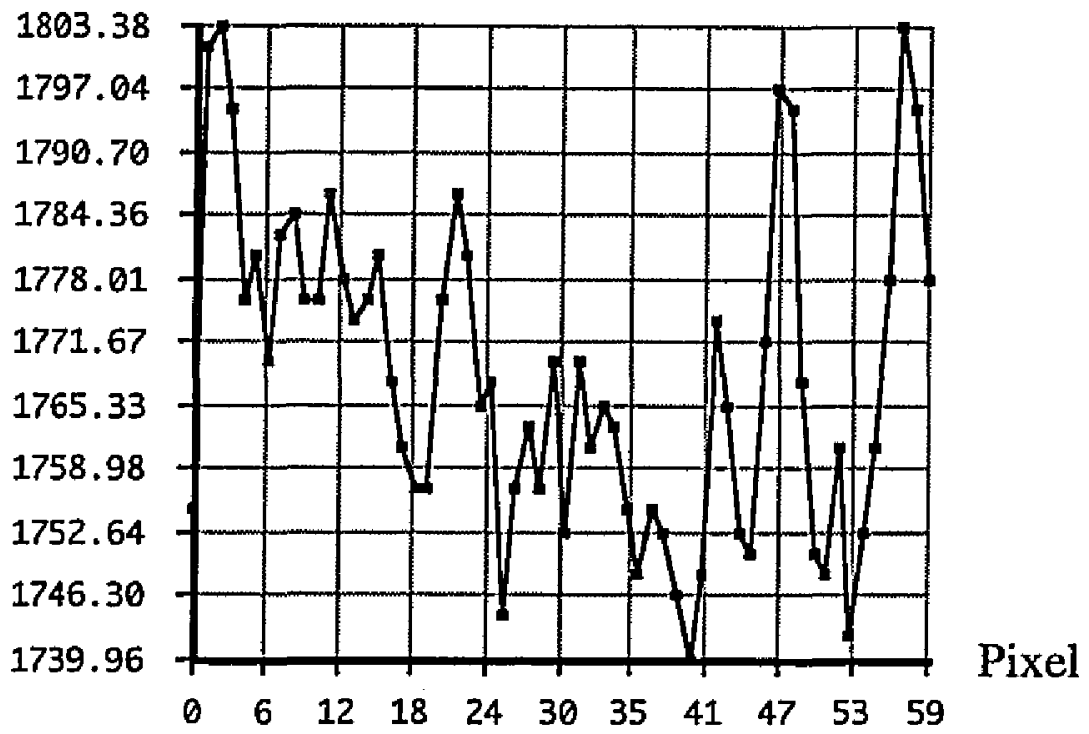

Fig. 5.5 (c) Column average for the fifth harmonic frequency domain image of specimen RBSN1.

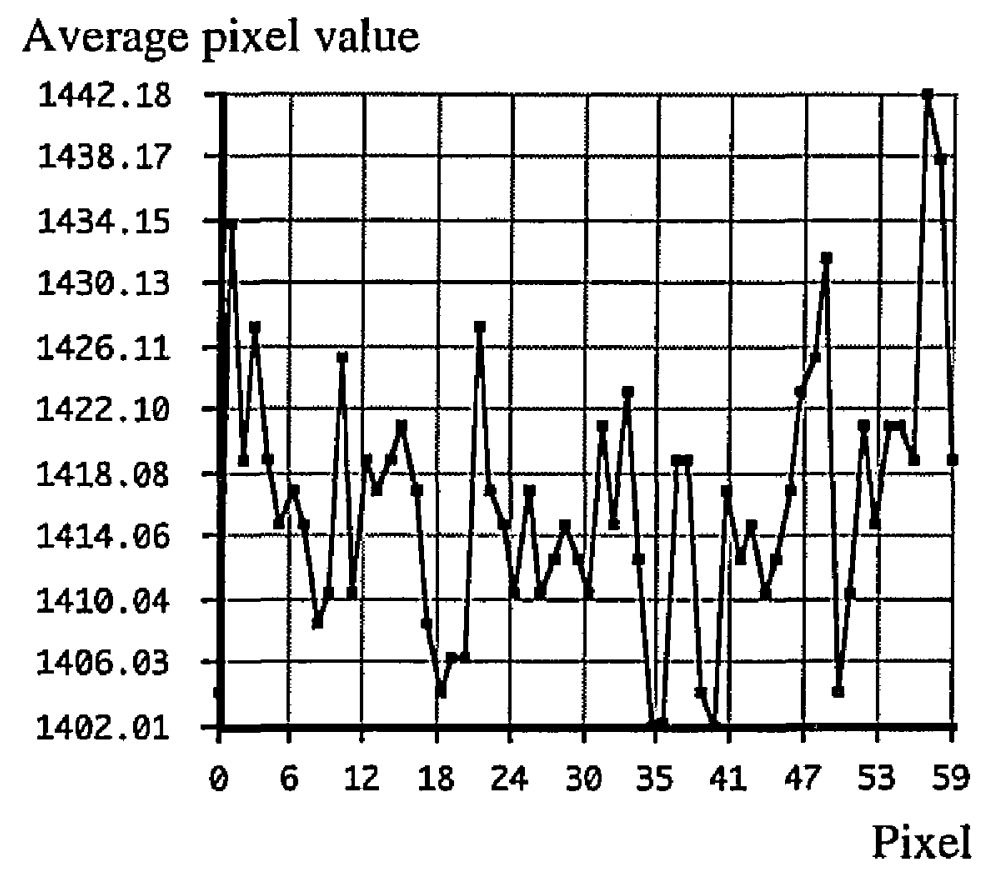

Fig. 5.5 (d) Column average for the seventh harmonic frequency domain image of specimen RBSN1. 
Average pixel value

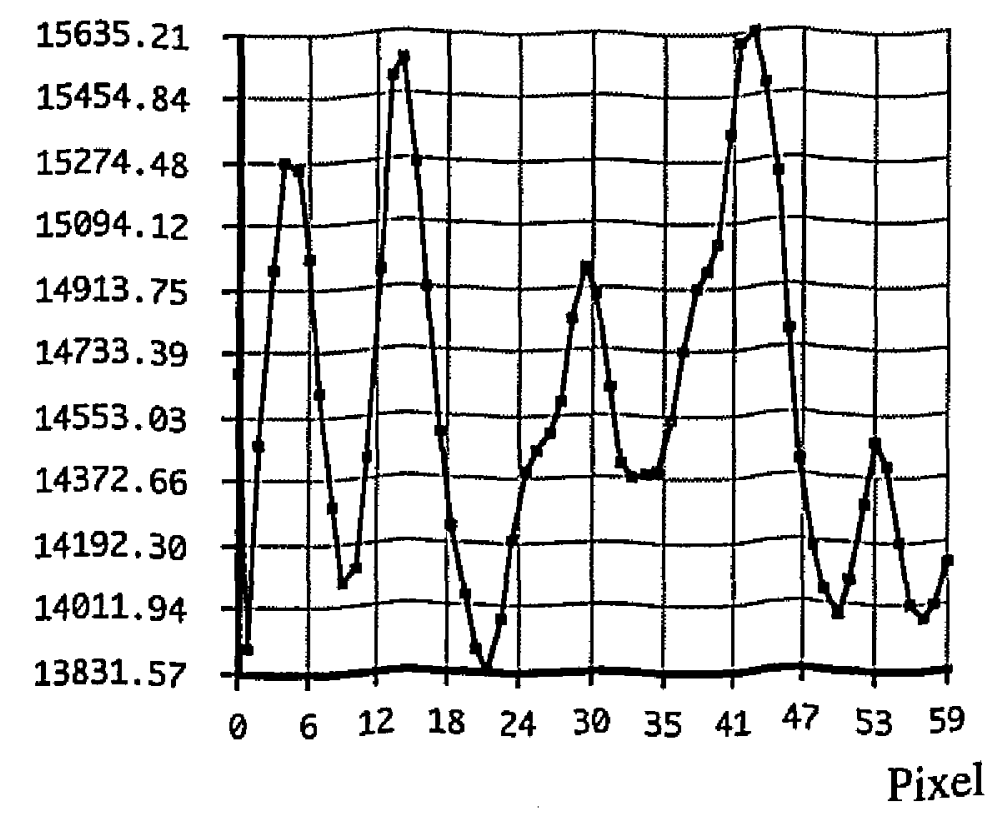

Fig. 5.6 (a) Column average for the first harmonic frequency domain image of specimen RBSN2.

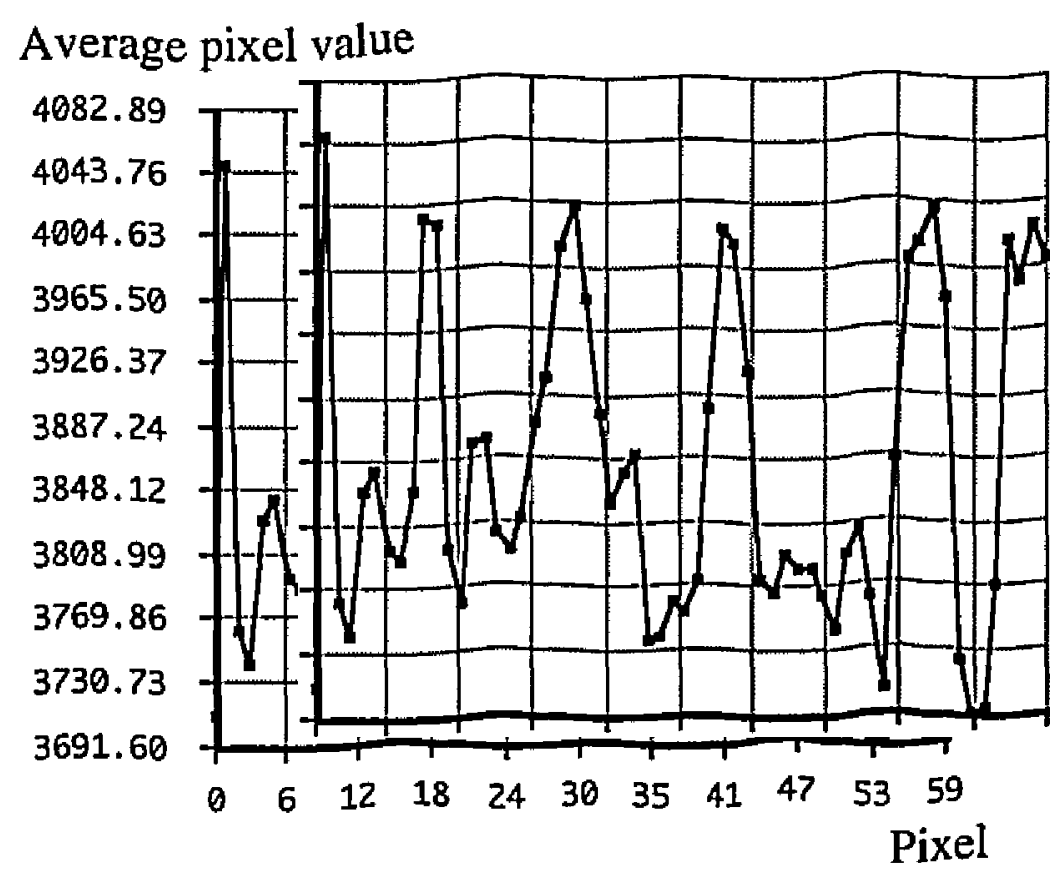

Fig. 5.6 (b) Column average for the third harmonic frequency domain image of specimen RBSN2. 
Average pixel value

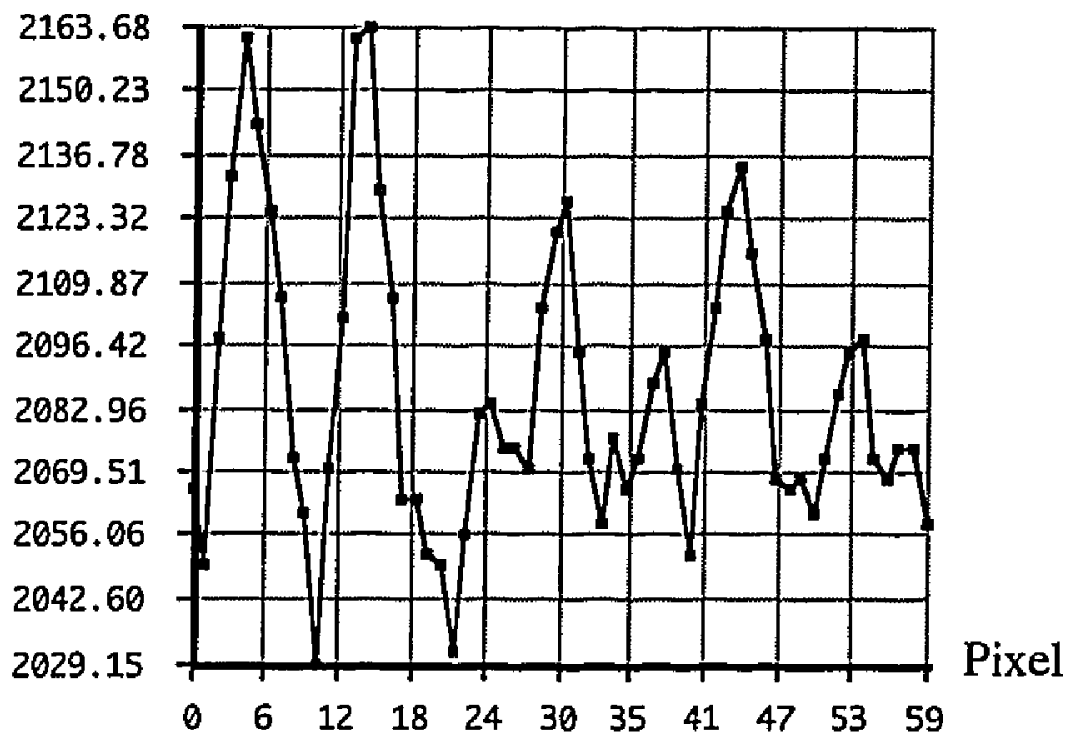

Fig. 5.6 (c) Column average for the fifth harmonic frequency domain image of specimen RBSN2.

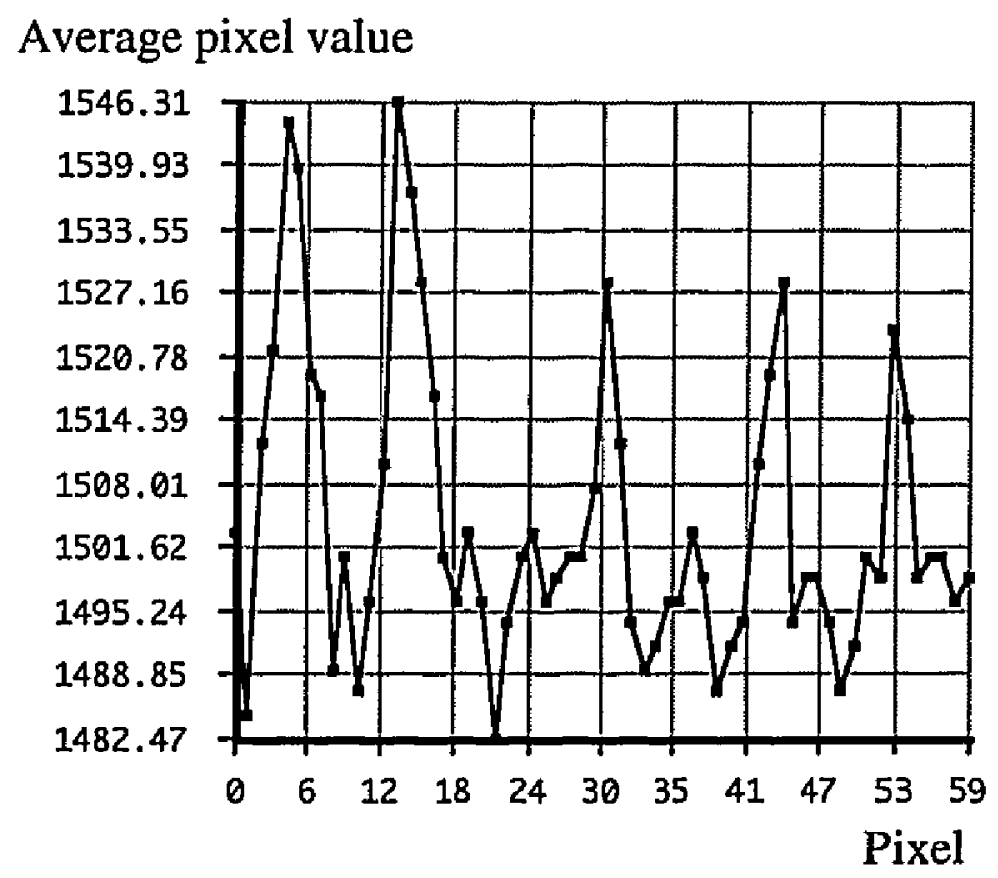

Fig. 5.6 (d) Column average for the seventh harmonic frequency domain image of specimen RBSN2. 


\section{CONCLUSION}

A study of the temporal thermal response was presented for flat multilayer structures and fiber reinforced composite materials. Models predicting the temperature response at the surface of such structures as a function of the combined responses of their constituents were derived. Techniques were developed to measure the thermal response of such structures. For flat multilayer geometries, the measurements were reduced to the thermal properties and compared to experimental data. For fiber reinforced composites, images of the fibers and subsurface defects were obtained.

In the case of multilayer structures, an analytical solution was developed for two and three layers. The method used to obtain the surface temperature as a function of the thermal properties of the layers is based on a expansion of the Laplace transforms of the solutions. For such geometries, solutions cannot be expressed in closed form expressions. Instead, they are found in the form of infinite series.

For two layers, the solution includes the first two terms of the expansions only. As a consequence, the first layer is assumed to be thermally thin compared to the second layer. The excitation frequency in the measurements must be tailored to the structure. For three layers all terms in the expansions are kept.

Measurements were made with a system that consisted of an infrared detector focused on a single point on the structures. An input heat flux was provided by either an Argon laser or a flash lamp depending on the configuration. The technique was successfully 
applied to the characterization of a thermally thin layer on a thermally thick layer. It was also applied to the characterization of the center layer of a three layer structure. Nonlinear least square fits of the models to the experimental data allowed to measure thickness and thermal properties. Results were found in good agreement with generally accepted values.

Measurements were also performed on fiber reinforced ceramic composite structures and a fabricated aluminum specimen. Images of the spatial variations in the thermal response were obtained by translation of the composite. To give insights in the significance of the data, a two dimensional analytical solution was developed for the thermal response of a fiber embedded in a matrix material. The solution was obtained by separation of variables of the heat equation expressed in cylindrical coordinates. The thermal response was assumed to be the sum of the temperature response due to the heat source and the scattered field off the fiber. For the fabricated aluminum specimen, the predicted temperature profile at the surface of the composite was found in good agreement with the experimental data. For the fiber reinforced ceramic composite specimens, the technique successfully imaged the fibers and thermal property changes due to subsurface thermally induced damages

The combination of thermal modeling with a data acquisition method that allows high temporal/spatial resolution and signal to noise ratio is a powerful tool for the nondestructive characterization of materials with complex geometries. It remains, in future work, to improve the models. In all simulations, the different materials were assumed to be in perfect thermal contact. However, this is not the case in many applications. Most riveted structures, damaged multilayered and fiber reinforced composite materials have a thermal response that exhibits an interface effect. As a first approximation, the interface regions 
can be modeled as a thin resistive layer. In the expansion method for flat multilayer walls, the Laplace transfer matrices take a simple form. But, if the layer is not thermally thin, its heat capacitance is non-zero. Then, the complexity increases and nonlinear least square fitting becomes difficult. This is due to the nature of the diffusion of heat in a solid. The attenuation factors involved are such that differentiating many layers becomes an impossible task.

For composite materials, it is possible to add a contact resistance at the interface region in the models. But more important are other effects to take into account: the 3 dimensional diffusion of heat in the direction of the fibers and the interactions between the scattered fields off many fibers. In the existing model, the heat source is assumed to be a line parallel to the fibers. In reality, the heat deposit made by the laser is a small spot. Adding another dimension to the model is a complex task. It involves expanding the heat source function in terms of spherical functions whereas the geometry of the composite material is best expressed in terms of cylindrical functions. Also, the model was developed for one fiber. The solution for many fibers can be obtained by superposition provided their spacing is large enough. In real composite specimens, they are too close to each other to obtain a realistic representation. The generalization to many fibers is a natural extension of the work presented here.

Another possibility for improvement in the thermal nondestructive characterization of materials is provided by a new generation of fast, more accurate infrared detectors. Focal point arrays allow temperature measurements in a much simpler fashion than the one used in this work. They permit to extend data acquisition to new structures and to better understand heat propagation phenomena in such materials. 


\section{APPENDIX A}

\section{THERMAL PROPERTIES OF SOLIDS}

There are two main mechanisms by which heat spreads through a solid:

- One involves the vibration of the crystal lattice and applies mainly to nonmetallic materials. It can also be important in some metals like Antimony or Bismuth, it will be presented in this appendix.

- The other involves electrons and is specific of metals. The carriers of heat, as well as electricity, in metals are the electrons. And, as one would expect, there is a close relationship between thermal and electrical conductivities. In fact, thermal conductivity can be deduced from the electrical conductivity by using the Wiedermann-FranzLorenz law. As most of the applications in industry do not require a very accurate determination of these values, the WFL law usually provides a quick and fairly good estimate. This useful relationship, though, does not take into account the interactions between the electrons and the crystal lattice. But, since a complete explanation of these phenomena is beyond the scope of this appendix, these interactions will not be discussed. 


\section{A Thermal properties of nonmetallic materials}

The mechanism by which heat is conducted in nonmetallic materials involves the thermal vibration of the atoms forming a wave motion. Because the atoms are coupled together, an increase of vibrational energy in one part of the lattice will be transmitted to the neighboring parts. In non-metals, it is the only or at least the dominant mean over a wide temperature range. The theory that considers nonmetallic crystals as vibrating mechanical lattices in which a heat flow results in a change of internal energy is the theory of phonons. It was developed in analogy with the quantum of electromagnetic radiation, the photon. The phonon is the quantum of vibrational energy. A full analysis of the heat flow due to lattice vibration being too complex to perform, some simplistic models were developed to account for the measurements of various thermal properties such as heat capacity and thermal conductivity.

\section{Lattice heat capacity}

Lattice heat capacity defines how much energy can be absorbed by a mechanical system. In fact, it is really a measure of the number of degrees of freedom of this system since each degree of freedom is a possibility to absorb potential or kinetic energy. As previously stated, a 3 dimensional crystal lattice is very difficult if not impossible to study in its full complexity. This explains why theories were developed after the experimental measurements were made rather than the contrary. These measurements showed that at room temperature range, the heat capacity of nearly all solids was a constant (approxi- 
mately $6 \mathrm{Cal} / \mathrm{K} \cdot \mathrm{mol}$, known as the Dulong and Petit value) and that, as the temperature $\mathrm{T}$ decreased, the heat capacity approached zero as $\mathrm{T}^{3}$.

\section{a Classical statistical mechanics}

For an ideal gas, the energy of a classical system is $\frac{k T}{2}$ per degree of freedom per particle where $\mathrm{T}$ is the absolute temperature and $\mathrm{k}$ the Boltzmann constant. For a set of particles with harmonic interactions, the total energy is the sum of the potential and kinetic energies. It turns out that they are equal and the total energy is $\mathrm{kT}$ per degree of freedom per particle.

In a 3 dimensional lattice composed of $\mathrm{N}$ atoms, each one of them being considered as an harmonic oscillator, the internal energy is $3 \mathrm{NkT}$ or $3 \mathrm{RT}$ for a mole, where $\mathrm{R}$ is Avogadro's number multiplied by $\mathrm{k}$.

The heat capacity is obtained by calorimetry and measured at constant pressure, but the value of heat capacity at constant volume is usually the same. Given the internal energy per unit mass, the heat capacity at constant volume is the derivative of the energy with respect to the temperature. Physically, the internal energy can be considered as the quantity of heat necessary to raise the temperature of a unit mass of material through the small temperature range $\mathrm{T}$ to $\mathrm{T}+\mathrm{dT}$. The expression for heat capacity is then:

$$
\mathrm{C}_{\mathrm{v}}=\frac{\partial \mathrm{U}}{\partial \mathrm{T}}
$$


From this, the value found corresponds to the Dulong and Petit value of approximately $6 \mathrm{Cal} / \mathrm{K} . \mathrm{mol}$.

Classical theory led to the simplest model for lattice heat capacity. The result above matches well the experimental measurements made in the room temperature range.

\section{b The Einstein model}

If classical statistical mechanics gives good results in the room temperature range, measurements show that, for low temperatures, the heat capacity approaches zero. To account for this tendency, the Einstein model sees the atoms as a set of independent oscillators vibrating with the same frequency $v$. According to Planck, the energy of an independent oscillator is quantized and has values nhv, where $\mathrm{n}$ is a positive integer and $\mathrm{h}$ is Planck's constant.

According to Boltzmann distribution law, the ratio of the populations in thermal equilibrium of two adjacent energy levels is:

$$
e^{-\frac{E_{n+1}-E_{n}}{k T}}=e^{-\frac{n h}{k T}}
$$

where $E_{n}$ is the energy at level $n$.

The average energy level is then given by:

$$
\overline{\mathrm{E}}=\frac{\sum_{n=0}^{\infty} n h v \mathrm{e}^{-\frac{\mathrm{nh} v}{\mathrm{kT}}}}{\sum_{\mathrm{n}=0}^{\infty} \mathrm{e}^{-\frac{\mathrm{nhv}}{\mathrm{kT}}}}=h v \frac{d}{d x} \log \left(\frac{1}{1-\mathrm{e}^{-\frac{\mathrm{hv}}{\mathrm{kT}}}}\right)
$$


where $\mathrm{x}=\mathrm{e}^{-\frac{\mathrm{h} v}{\mathrm{kT}}}$. Finally,

$$
\bar{E}=\frac{h v}{e^{-\frac{h v}{k T}}-1}
$$

The validity of Einstein model can be checked:

- For high temperatures when $k T » h v$, that gives $e^{-\frac{h v}{k T}}-1 \approx \frac{h v}{k T}$ and $\bar{E} \approx k T$ which correspond to the Dulong and Petit value of the classical mechanics.

- For low temperatures, when $k T \ll h v$, then $e^{-\frac{h v}{k T}} \ll 1$ and $\bar{E} \approx h v\left(1+e^{-\frac{h v}{k T}}\right)$.

The heat capacity for $\mathrm{N}$ atoms that corresponds to the average energy above is:

$$
C_{v}=\frac{\partial \bar{E}}{\partial T}=N k\left(\frac{h v}{k T}\right)^{2} e^{-\frac{h v}{k T}}
$$

As $\mathrm{T}$ approaches zero, the exponential term is dominant and, as a consequence, $\mathrm{C}_{\mathrm{v}}$ approaches zero as $\mathrm{e}^{-\frac{\mathrm{hv}}{\mathrm{kT}}}$.

In the Einstein model, the lattice heat capacity decays exponentially to zero as ' $T$ approaches zero. Considering the atoms as a set of independent oscillators led to a better approximation of the general behavior of the heat capacity but experiments were precise enough to reveal that the decrease was proportional to $\mathrm{T}^{3}$. Debye developed a better model to account for this proportionality. 


\section{c The Debye model}

According to the phonon theory, the atoms oscillate at a given frequency relative to their neighbors. Assigning the same frequency to all $3 \mathrm{~N}$ oscillators (Einstein model) is an over-simplification in the low temperature range. Debye model accounts for these variations in frequency. Since the derivation leading to the approximation of the lattice heat capacity is long and strenuous, it will only be overviewed here.

Debye considered that, of the $3 \mathrm{~N}$ modes of vibration of the crystal lattice, there were $C(\omega) d \omega$ modes in the frequency range $d \omega$ at $\omega$. Then, the total lattice energy can be written as:

$$
\mathrm{U}=\int_{0}^{\omega_{\mathrm{m}}} \frac{h \omega C(\omega) \mathrm{d} \omega}{2 \pi\left(\mathrm{e}^{\frac{h \omega}{2 \pi \mathrm{k}^{\top} \mathrm{T}}}-1\right)}
$$

where $\omega_{m}$ is the cut-off frequency. After changing the variables, $U$ becomes:

$$
U=\frac{3 k^{4} T^{4}}{16 \pi^{5} h v_{0}^{3}} \int_{0}^{x_{n+1}} \frac{x^{3} d x}{e^{x}-1}
$$

where $x_{m}=\frac{h \omega_{m}}{2 \pi k T}=\frac{\Theta}{T}$ with $\Theta$ as the Debye characteristic temperature and $v_{0}$ as the mean phonon velocity (approximately the velocity of sound in the crystal).

As previously, the heat capacity is given by taking the derivative of the energy U. That gives: 


$$
C_{v}=9 N k\left(\frac{T}{\Theta}\right)^{3} \int_{0}^{x_{m}} \frac{e^{x} x^{4} d x}{\left(e^{x}-1\right)^{2}}
$$

For $T » \Theta$, the heat capacity approaches the Dulong and Petit value of $3 \mathrm{R} / \mathrm{mole}$.

At very low temperatures, the integral in Eq. (7) becomes $\int_{0}^{\infty} \frac{\mathrm{x}^{3} \mathrm{dx}}{\mathrm{e}^{\mathrm{x}}-1}=\frac{\pi^{4}}{15}$.

Therefore, the internal energy is:

$$
U=\frac{3}{5} \pi^{4} \mathrm{Nk} \frac{\mathrm{T}^{4}}{\Theta^{3}}
$$

The heat capacity now exhibits the $\mathrm{T}^{3}$ behavior:

$$
C_{v}=\frac{12}{5} \pi^{4} \mathrm{Nk}\left(\frac{\mathrm{T}}{\Theta}\right)^{3}
$$

In the Debye approximation, the key is the determination of the Debye temperature $\Theta$, which in turn involves the measurement of the velocity of sound in a given crystal. Some values for $\Theta$ are available in Blackman's tables. Fig. A.1 shows $C_{v}$ according to Debye's theory. 


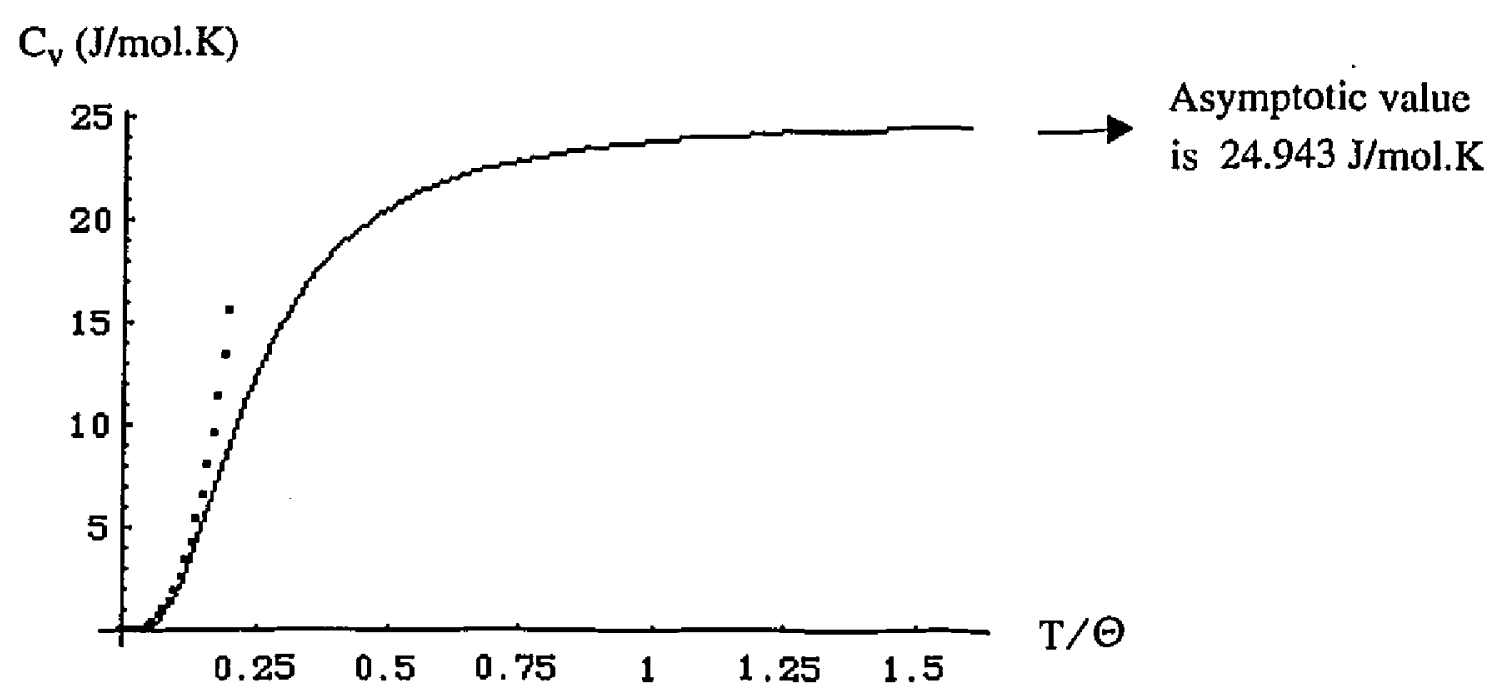

Fig. A.1 Heat capacity of a solid according to the Debye approximation. The vertical scale is in $\mathrm{J} / \mathrm{mol}$. K. The horizontal scale is the temperature normalized to the Debye temperature $\Theta$. The region of the $\mathrm{T}^{3}$ law, below $0.2 \Theta$, is shown in dots. The asymptotic value at high temperatures is the Dulong and Petit constant.

\section{Thermal conductivity}

Heat conduction is a result of a change from the equilibrium state in a material.

According to the principles of thermodynamics, a heat flow occurs from a region where energy is concentrated to regions with lower energies. The thermal conductivity is introduced in the relationship:

$$
\mathrm{Q}=\mathrm{K} \frac{d \mathrm{~T}}{d \mathrm{x}}
$$

where $\mathrm{Q}$ is the flux, $\mathrm{K}$ the thermal conductivity and $\mathrm{x}$ the position on the axis along which the flux occurs. 
The definition above is general and applies to nonmetallic materials as well as to metals. It is the relationship that is used to perform the conductivity measurements. Furthermore, if we assume that energies are transferred by exchange between the different phonon modes, conductivity can be defined as:

$$
\mathrm{K}=\frac{1}{3} \mathrm{C}_{\mathrm{v}} \mathrm{v}_{0} 1
$$

where $v_{0}$ is the velocity of sound in the crystal, $C_{v}$ the lattice heat capacity and $l$ the mean free path. The mean free path is the distance such a wave travels before its intensity is attenuated by scattering to $1 / \mathrm{e}$ of its initial value. Practically, it is a measure of the rate at which heat is exchanged.

Obviously, since $v_{0}$ is a constant of the crystal lattice, the behavior of heat conductivity depends on the variations of $l$ and $C_{v}$ with temperature. The dependence of the heat capacity was explained: for temperatures that are high enough $C_{v}$ is a constant and, as temperature drops down to zero, $C_{v}$ behaves like $T^{3}$.

For sufficiently high temperatures, 1 is proportional to $1 / T$ and, as $T$ decreases to very low temperatures, I tends to a constant value which depend on the geometry of the specimen.

The combination of the two behaviors produces the typical curve for nonmetallic materials as in Fig. A.2. 
Thermal conductivity

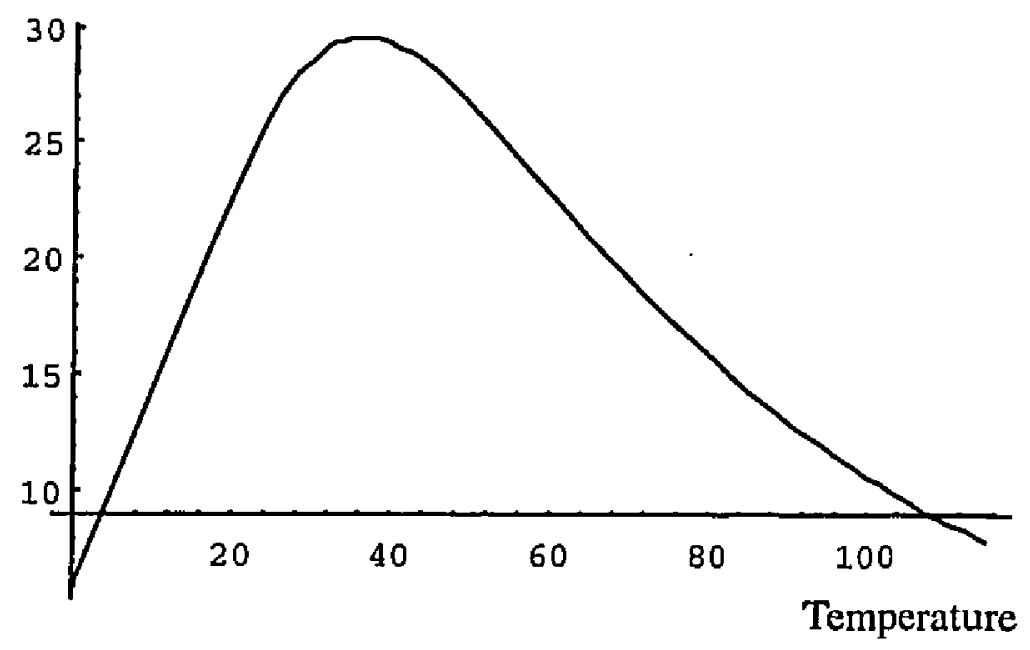

Fig. A.2 Thermal conductivity of nonmetallic material for typical values used in Fig. A.1.

\section{B Thermal properties of metallic materials}

In metals, heat is mainly transported by electrons. Typically, the electrons, which are free to move, can be accelerated by a temperature gradient and will travel through a metal until they hit an atom and transfer their excess thermal energy. This mechanism of heat

conduction can be dominant, like in Copper or Silver, or, as in Antimony or Bismuth, be of equal importance as lattice conduction.

Quantum theory results for heat capacity associated with electronic conduction are presented first. Then, the thermal conductivity due to electrons as well as a derivation of Wiedermann-Franz-Lorenz law that provides a good estimate of the thermal conductivity of a metal given its electrical conductivity are discussed. 


\section{Heat capacity of conduction electrons}

For free electrons of mass $m_{e}$, the quantum theory result is that the electronic heat capacity is given by:

$\mathrm{C}_{\mathrm{v}}$ (electrons) $=\gamma \mathrm{T} \quad$ where $\gamma$ is of the order of $10^{-4} \mathrm{Cal} / \mathrm{mol} \mathrm{K}^{2}$

We note that $\mathrm{C}_{\mathrm{v}}$ (electrons) is linear in $\mathrm{T}$.

\section{Thermal conductivity \& the WFL law}

Thermal conductivity varies with the type of metal. In pure metals, electronic conduction is the dominant mode whereas, in impure metals or alloys, collisions between electrons and phonons become important and the lattice contribution to conductivity becomes comparable to the electronic contribution.

In the case of pure metals, the Wiedermann-Franz-Lorenz law establishes a useful relationship between electrical and thermal conductivities.

The electrical conductivity can be expressed as:

$$
\sigma=\frac{\mathrm{n}_{\mathrm{e}} \mathrm{e}^{2} 1}{2 \mathrm{~m}_{\mathrm{e}} \mathrm{v}}
$$

where $v$ is the number of free electrons per unit volume, $e, m_{e}$ and $n_{e}$ are their electric charge, mass and number, and $v$ their thermal velocity.

The thermal conductivity is given by: 


$$
\mathrm{K}=\frac{1}{3} \mathrm{n}_{\mathrm{e}} \mathrm{C}_{\mathrm{e}} \mathrm{vI}
$$

where $C_{c}$ is the heat capacity per electron.

The ratio of the thermal to the electrical conductivities gives the Wiedermann-FranzLorenz law:

$$
\frac{K}{\sigma}=\frac{2 m_{e} C_{e} v^{2}}{3 e^{2}}
$$

From a classical point of view, the free electrons are assumed to be Fermi gas-like particles. Then, $\mathrm{C}_{\mathrm{e}}$ is a constant and $\mathrm{v}^{2}$ is proportional to $\mathrm{T}$.

From a quantum mechanical point of view, $v$ does not depend on temperature and $C_{c}$ is proportional to $\mathrm{T}$. That gives:

$$
\frac{\mathrm{K}}{\sigma}=\frac{\pi^{2} \mathrm{k}^{2}}{3 \mathrm{e}^{2}} \mathrm{~T}
$$

Both points of view lead to the WFL law which states that:

$$
\frac{\mathrm{K}}{\sigma \mathrm{T}}=\mathrm{cons} \tan \mathrm{t}
$$

Even though WFL law is only an approximation, it is very useful because, from the electrical conductivity, which is experimentally easy to determine, values for the thermal conductivity can be obtained. 


\section{APPENDIX B}

\section{DETAILED DERIVATIONS FOR APPROXIMATED TWO LAYER MODEL}

\section{A Notations}

In the following discussions, the multilayer structure is represented by a one dimensional model. Layer $\mathrm{n}$ has thickness $\mathrm{l}_{\mathrm{n}}$, thermal diffusivity $\alpha_{n}$, thermal conductivity $K_{n}$ and is in perfect thermal contact with adjacent layers. Convection losses at the first and last layers are neglected. $t$ is the time, $p$ the Laplace variable and $q_{n}=\sqrt{p / \alpha_{n}}$.

\section{B Inversion of the first order approximation solution}

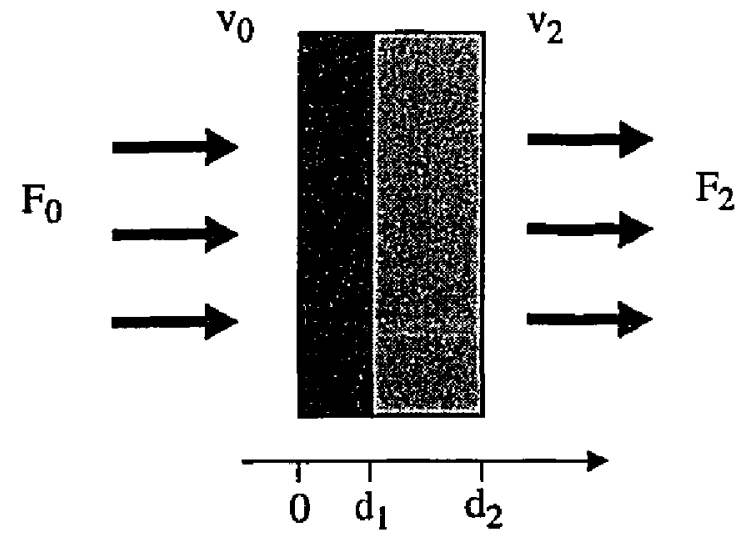

Fig. B.1 Model for a two layer structure. 
For a two layer structure, the expansion of $\bar{v}_{0}$ is performed about $q_{1}$ equal to zero. The transfer matrix for the first layer takes the form:

$$
\mathrm{T}_{1}=\left[\begin{array}{cc}
1+\mathrm{O}\left(\mathrm{q}_{1}^{2}\right) & -\left(\frac{\mathrm{l}_{1}}{\mathrm{~K}_{1}}\right)+\mathrm{O}\left(\mathrm{q}_{1}^{2}\right) \\
\mathrm{O}\left(\mathrm{q}_{1}^{2}\right) & 1+\mathrm{O}\left(\mathrm{q}_{1}^{2}\right)
\end{array}\right]
$$

where $l_{1}=d_{1}-0$ is the thickness of the first layer.

Multiplication with the full form for $\mathrm{T}_{2}$ gives:

$\left[\begin{array}{ll}A & B \\ C & D\end{array}\right]=\left[\begin{array}{cc}\cosh \left(l_{2} q_{2}\right)+O\left(q_{1}^{2}\right) & -\left(\frac{l_{1} \cosh \left(l_{2} q_{2}\right)}{K_{1}}+\frac{\sinh \left(l_{2} q_{2}\right)}{K_{2} q_{2}}\right)+O\left(q_{1}^{2}\right) \\ -K_{2} q_{2} \sinh \left(l_{2} q_{2}\right)+O\left(q_{1}^{2}\right) & \cosh \left(l_{2} q_{2}\right)+\frac{K_{2} I_{1} q_{2} \sinh \left(l_{2} q_{2}\right)}{K_{1}}+O\left(q_{1}^{2}\right)\end{array}\right]$

where $l_{2}=d_{2}-d_{1}$.

The Laplace transform of the temperature due to a constant flux starting at $t=0$ with no flux at the back surface is evaluated by taking the ratio $-\mathrm{D} / \mathrm{Cp}$ :

$$
\overline{v_{0}}=F_{0}\left(\frac{l_{1}}{\mathrm{~K}_{1} \mathrm{p}}+\frac{\operatorname{coth}\left(\mathrm{l}_{2} \mathrm{q}_{2}\right)}{\mathrm{K}_{2} \mathrm{q}_{2} \mathrm{p}}\right)+\mathrm{O}\left(\mathrm{q}_{1}^{2}\right)
$$

Inversion of $\frac{\mathrm{l}_{1}}{\mathrm{~K}_{1} \mathrm{p}}$ is trivial. $\frac{\mathrm{l}_{1} \mathrm{~F}_{0}}{\mathrm{~K}_{1}}$ represents a temperature increase directly proportional to the incoming heat flux. It is due to the first layer, modeled as a contact resistance. Inversion of $\frac{\operatorname{coth}\left(\mathrm{l}_{2} \mathrm{q}_{2}\right)}{\mathrm{K}_{2} \mathrm{q}_{2} \mathrm{p}}$ can be performed by expressing the hyperbolic functions in terms of negative exponential functions, and by expanding them into an infinite series. 
Then, to the first order:

$\overline{v_{0}}=F_{0}\left(\frac{l_{1}}{K_{1} p}+\frac{\left(e^{l_{2} q_{2}}+e^{-l_{2} q_{2}}\right)}{K_{2} q_{2} p e^{l_{2} q_{2}}\left(1-e^{-2 l_{2} q_{2}}\right)}\right)=F_{0}\left(\frac{l_{1}}{K_{1} p}+\frac{1}{K_{2} q_{2} p}\left(1+2 \sum_{n=1}^{\infty} e^{-2 n l_{2} q_{2}}\right)\right)$

$\bar{v}_{0}$ is inverted term by term from standard tables of transforms. $v_{0}$ to the first order is:

$$
v_{0}=F_{0}\left(\frac{1_{1}}{K_{1}}+\frac{2 \sqrt{\alpha_{2} t}}{K_{2}} \sum_{n=0}^{\infty}\left(\operatorname{ierfc}\left(\frac{n_{2}}{\sqrt{\alpha_{2} t}}\right)+\operatorname{ierfc}\left(\frac{(n+1) l_{2}}{\sqrt{\alpha_{2} t}}\right)\right)\right)
$$

where ierfe is the intergral complementary Error function:

$$
\operatorname{ierfc}(x)=\frac{e^{-x^{2}}}{\sqrt{\pi}}-x(\operatorname{Erfc}(x))
$$

\section{Inversion of the third order approximation solution}

The transfer matrix for the first layer takes the form:

$$
T_{1}=\left[\begin{array}{cc}
1+\frac{1_{1}^{2} q_{1}^{2}}{2}+O\left(q_{1}^{4}\right) & -\frac{1_{1}}{K_{1}}-\frac{1_{1}^{3} q_{1}^{2}}{6 K_{1}}+O\left(q_{1}^{4}\right) \\
-K_{1} l_{1} q_{1}+O\left(q_{1}^{4}\right) & 1+\frac{1_{1}^{2} q_{1}^{2}}{2}+O\left(q_{1}^{4}\right)
\end{array}\right]
$$

Multiplication with the full form for $\mathrm{T}_{2}$ yields $\mathrm{A}, \mathrm{B}, \mathrm{C}$ and $\mathrm{D}$ : 


$$
\begin{aligned}
& A=\cosh \left(l_{2} q_{2}\right)+\left(\frac{l_{1}^{2} \cosh \left(I_{2} q_{2}\right)}{2}+\frac{K_{1} l_{1} \sinh \left(l_{2} q_{2}\right)}{K_{2} q_{2}}\right) q_{1}^{2}+O\left(q_{1}^{4}\right) \\
& B=-\left(\frac{l_{1} \cosh \left(l_{2} q_{2}\right)}{K_{1}}+\frac{\sinh \left(l_{2} q_{2}\right)}{K_{2} q_{2}}\right)-\left(\frac{l_{1}^{3} \cosh \left(l_{2} q_{2}\right)}{6 K_{1}}+\frac{l_{1}^{2} \sinh \left(l_{2} q_{2}\right)}{2 K_{2} q_{2}}\right) q_{1}^{2}+O\left(q_{1}^{4}\right) \\
& C=-K_{2} q_{2} \sinh \left(l_{2} q_{2}\right)-\left(K_{1} l_{1} \cosh \left(l_{2} q_{2}\right)+\frac{K_{2} l_{1}^{2} q_{2} \sinh \left(l_{2} q_{2}\right)}{2}\right) q_{1}^{2}+O\left(q_{1}^{4}\right) \\
& D=\cosh \left(l_{2} q_{2}\right)+\frac{K_{2} l_{1} q_{2} \sinh \left(l_{2} q_{2}\right)}{K_{1}}+\left(\frac{l_{1}^{2} \cosh \left(l_{2} q_{2}\right)}{2}+\frac{K_{2} l_{1}^{3} q_{2} \sinh \left(l_{2} q_{2}\right)}{6 K_{1}}\right) q_{1}^{2}+O\left(q_{1}^{4}\right)
\end{aligned}
$$

The Laplace transform of the temperature due to a constant flux starting at $t=0$ with no flux at the back surface is evaluated by taking the ratio $-\mathrm{D} / \mathrm{Cp}$. After simplification, the ratio can be expressed as the sum of the first order approximation and of higher order terms:

$$
\overline{v_{0}}=F_{0}\left(\frac{l_{1}}{K_{1} p}+\frac{\operatorname{coth}\left(l_{2} q_{2}\right)}{K_{2} q_{2} p}-\frac{1_{1}^{3} q_{1}^{2}}{3 K_{1} p}-\frac{1_{1}^{2} q_{1}^{2} \operatorname{coth}\left(l_{2} q_{2}\right)}{K_{2} q_{2} p}-\frac{K_{1} l_{1} q_{1}^{2} \operatorname{coth}^{2}\left(l_{2} q_{2}\right)}{K_{2}^{2} q_{2}^{2} p}\right)+O\left(q_{1}^{4}\right)
$$

Each one of the higher order terms is simple enough to be inverted by the standard methods.

\section{Inversion of the first higher order term}

Inversion of the first higher order term can be peformed from standard tables of transform:

$$
\mathrm{L}^{-1}\left(\frac{\mathrm{l}_{1}^{3} \mathrm{q}_{1}^{2}}{3 \mathrm{~K}_{1} \mathrm{p}}\right)=\frac{\mathrm{l}_{1}^{3}}{3 \mathrm{~K}_{1} \alpha_{1}^{2}} \delta(\mathrm{t})
$$


where $\delta(t)$ is the delta function. It cannot be represented in the simulations and will be removed from the final model.

\section{Inversion of the second higher order term}

a Inversion by use of the Inversion Theorem for the Laplace transformation

The inverse Laplace transform is given by:

$$
L^{-1}\left(\frac{1_{1}^{2} q_{1}^{2} \operatorname{coth}\left(l_{2} q_{2}\right)}{K_{2} q_{2} p}\right)=\frac{1}{2 \pi i} \frac{\alpha_{2} 1_{1}^{2}}{\alpha_{1} K_{2} \sqrt{\alpha_{2}}} \int_{\gamma-i \infty}^{\gamma+i \infty} \frac{e^{\lambda t} \cosh \left(l_{2} \mu\right)}{\sqrt{\lambda} \sinh \left(1_{2} \mu\right)} d \lambda
$$

where $\lambda=\alpha_{2} \mu^{2}$.

$\gamma$ is to be large enough so that all singularities of $\cosh \left(1_{2} \mu\right) /\left(\sqrt{\lambda} \sinh \left(1_{2} \mu\right)\right)$ lie to the left of the line $(\gamma-j \infty, \gamma+i \infty)$. The line integral can be evaluated by completing the contour by a large circle $\Gamma$ such as shown in Fig. B.2. 


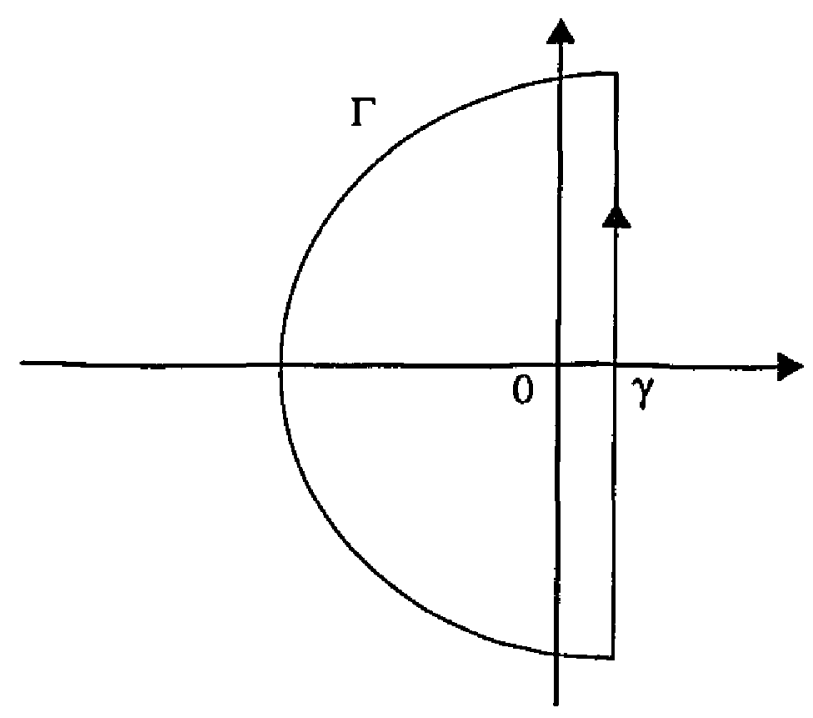

Fig. B.2 Contour integral used for the inverse Laplace transform.

The integral over the large circle $\Gamma$ vanishes when its radius tends to $\infty$. In the limit, the line integral is equal to $2 \pi \mathrm{i}$ times the sum of the residues at the poles of the integrand. There are poles at the values of $\lambda$ that make $\sinh \left(1_{2} \mu\right)=0$ and at $\lambda=0$.

$\sinh \left(l_{2} \mu\right)=0 \Rightarrow \mu=\frac{n \pi i}{l_{2}}$ with $n=1,2,3 \ldots$

which leads to the values of $\lambda: \lambda=-\left(\frac{\mathrm{n} \pi}{\mathrm{l}_{2}}\right)^{2} \alpha_{2}$ with $\mathrm{n}=1,2,3 \ldots$

The residues are: $\mathrm{e}^{\lambda t} \cosh \left(\mathrm{l}_{2} \mu\right) /\left(\frac{d}{d \lambda} \sqrt{\lambda} \sinh \left(\mathrm{I}_{2} \mu\right)\right)$ for $\lambda=-\left(\frac{\mathrm{n} \pi}{\mathrm{l}_{2}}\right)^{2} \alpha_{2}$ with $\mathrm{n}=1$, $2,3 \ldots$

They simplify to: $\frac{2 \sqrt{\alpha_{2}} e^{-\frac{n^{2} \pi^{2} \alpha_{2} \mathrm{t}}{l_{2}^{2}}}}{l_{2}}$ with $n=1,2,3 \ldots$ 
The residue at zero is obtained by performing a Laurent expansion and by taking the coefficient of the $1 / \mu$ term: $\sqrt{\alpha_{2}} / 1_{2}$

The part due to this higher order term is:

$$
L^{-1}\left(\frac{I_{1}^{2} q_{1}^{2} \operatorname{coth}\left(l_{2} q_{2}\right)}{K_{2} q_{2} p}\right)=\frac{l_{1}^{2} \alpha_{2}}{\alpha_{1} K_{2} l_{2}}\left(1+2 \sum_{n=1}^{\infty} e^{-\frac{n^{2} \pi^{2} \alpha_{2} t}{l_{2}^{2}}}\right)
$$

\section{b Inversion by expansion method}

Inversion can be performed by expressing the hyperbolic functions in terms of negative exponential functions and by expanding the ratio into an infinite series:

$$
\begin{aligned}
& \frac{\mathrm{l}_{1}^{2} \mathrm{q}_{1}^{2} \operatorname{coth}\left(\mathrm{l}_{2} \mathrm{q}_{2}\right)}{\mathrm{K}_{2} \mathrm{q}_{2} \mathrm{p}}=\frac{\mathrm{l}_{1}^{2} \mathrm{q}_{2} \alpha_{2}}{\alpha_{1} \mathrm{~K}_{2} \mathrm{p}}\left(\frac{\mathrm{e}^{\mathrm{l}_{2} \mathrm{q}_{2}}+\mathrm{e}^{-\mathrm{l}_{2} \mathrm{q}_{2}}}{\mathrm{e}^{\mathrm{l}_{2} \mathrm{q}_{2}}-\mathrm{e}^{-\mathrm{l}_{2} \mathrm{q}_{2}}}\right)=\frac{\mathrm{l}_{1}^{2} \mathrm{q}_{2} \alpha_{2}}{\alpha_{1} \mathrm{~K}_{2} \mathrm{p}}\left(\frac{1+\mathrm{e}^{-2 \mathrm{l}_{2} \mathrm{q}_{2}}}{1-\mathrm{e}^{-2 \mathrm{l}_{2} \mathrm{q}_{2}}}\right) \\
& =\frac{\mathrm{l}_{1}^{2} \mathrm{q}_{2} \alpha_{2}}{\alpha_{1} \mathrm{~K}_{2} \mathrm{p}}\left(1+\mathrm{e}^{-2 \mathrm{l}_{2} \mathrm{q}_{2}}\right) \sum_{\mathrm{n}=0}^{\infty} \mathrm{e}^{-2 \mathrm{nq}_{2} \mathrm{l}_{2}}
\end{aligned}
$$

The expression needs to be arranged into a sum of terms with a known inverse.

$$
\frac{1_{1}^{2} q_{2} \alpha_{2}}{\alpha_{1} K_{2} p} \sum_{n=0}^{\infty}\left(e^{-2 n q_{2} l_{2}}+e^{-2(n+1) q_{2} l_{2}}\right)=\frac{1_{1}^{2} \alpha_{2}}{\alpha_{1} K_{2}} \sum_{n=0}^{\infty}\left(\frac{e^{-2 n \sqrt{\frac{p}{\alpha_{2}}} l_{2}}}{\sqrt{\alpha_{2} p}}+\frac{e^{-2(n+1) \sqrt{\frac{p}{\alpha_{2}}} l_{2}}}{\sqrt{\alpha_{2} p}}\right)
$$

The inverse Laplace transform is then taken term by term from standard tables:

$$
\mathrm{L}^{-1}\left(\frac{\mathrm{l}_{1}^{2} \mathrm{q}_{1}^{2} \operatorname{coth}\left(\mathrm{l}_{2} \mathrm{q}_{2}\right)}{\mathrm{K}_{2} \mathrm{q}_{2} \mathrm{p}}\right)=\frac{\mathrm{l}_{1}^{2} \alpha_{2}}{\alpha_{1} \mathrm{~K}_{2} \sqrt{\pi \alpha_{2} \mathrm{t}}} \sum_{n=0}^{\infty}\left(\mathrm{e}^{-\frac{\left.\mathrm{n}^{2}\right]_{2}^{2}}{\alpha_{2} \mathrm{t}}}+\mathrm{e}^{-\frac{(\mathrm{n}+1)^{2} l_{2}^{2}}{\alpha_{2} \mathrm{l}}}\right)
$$




\section{Inversion of the third higher order term}

\section{a Inversion by Inversion Theorem for the Laplace transformation}

The inverse Laplace transform is given by:

$$
L^{-1}\left(\frac{1_{1} K_{1} q_{1}^{2} \operatorname{coth}^{2}\left(l_{2} q_{2}\right)}{K_{2}^{2} q_{2}^{2} p}\right)=\frac{1}{2 \pi i} \frac{\alpha_{2} l_{1} K_{1}}{\alpha_{1} K_{2}^{2}} \int_{\gamma-i \infty}^{\gamma+i \infty} \frac{e^{\lambda t} \cosh ^{2}\left(l_{2} \mu\right)}{\lambda \sinh ^{2}\left(l_{2} \mu\right)} d \lambda
$$

where $\lambda=\alpha_{2} \mu^{2}$.

As for the second higher order term, the integration is performed by completing the contour by a large circle $\Gamma$ such as shown in Fig. B.2. The integral over the large circle $\Gamma$ vanishes when its radius tends to $\infty$ and, in the limit, the line integral is equal to $2 \pi i$ times the sum of the residues at the poles of the integrand.

There are poles at the values of $\lambda$ that make $\sinh \left(I_{2} \mu\right)=0$ and at $\lambda=0$.

$$
\sinh \left(1_{2} \mu\right)=0 \Rightarrow \lambda=-\left(\frac{\mathrm{n} \pi}{\mathrm{l}_{2}}\right)^{2} \alpha_{2} \text { with } \mathrm{n}=1,2,3 \ldots
$$

The corresponding residues are: $\mathrm{e}^{\lambda t} \cosh ^{2}\left(\mathrm{I}_{2} \mu\right) /\left(\frac{d^{2}}{d \lambda^{2}} \lambda \sinh ^{2}\left(\mathrm{I}_{2} \mu\right)\right)$

for $\lambda=-\left(\frac{n \pi}{I_{2}}\right)^{2} \alpha_{2}$ with $\mathrm{n}=1,2,3 \ldots$

They simplify to: $\frac{2 \alpha_{2} \mathrm{e}^{-\frac{\mathrm{n}^{2} \pi^{2} \alpha_{2} \mathrm{t}}{l_{2}^{2}}}}{1_{2}^{2}}$ with $\mathrm{n}=1,2,3 \ldots$

The residue at zero is obtained by Laurent expansion: $\frac{\alpha_{2} t}{l_{2}^{2}}+\frac{2}{3}$

The part due to this higher order term is: 


$$
L^{-1}\left(\frac{l_{1} K_{1} q_{1}^{2} \operatorname{coth}^{2}\left(l_{2} q_{2}\right)}{K_{2}^{2} q_{2}^{2} p}\right)=\frac{l_{1} K_{1} \alpha_{2}}{\alpha_{1} K_{2}^{2}}\left(\frac{2}{3}+\frac{\alpha_{2}}{l_{2}^{2}}\left(t+\sum_{n=1}^{\infty} 2 e^{-\frac{n^{2} \pi^{2} \alpha_{2} t}{l_{2}^{2}}}\right)\right)
$$

\section{b Inversion by expansion method}

Inversion can be performed by expressing the hyperbolic functions in terms of negative exponential functions and by expanding the ratio into an infinite series:

$$
\begin{aligned}
& \frac{l_{1} K_{1} q_{1}^{2} \operatorname{coth}^{2}\left(l_{2} q_{2}\right)}{K_{2}^{2} q_{2}^{2} p}=\frac{l_{1} K_{1} \alpha_{2}}{\alpha_{1} K_{2}^{2}} \frac{\cosh ^{2}\left(l_{2} q_{2}\right)}{\sinh ^{2}\left(l_{2} q_{2}\right)}=\frac{l_{1} K_{1} \alpha_{2}}{\alpha_{1} K_{2}^{2} p}\left(\frac{e^{l_{2} q_{2}}+e^{-l_{2} q_{2}}}{e^{l_{2} q_{2}}-e^{-l_{2} q_{2}}}\right)^{2} \\
& =\frac{l_{1} K_{1} \alpha_{2}}{\alpha_{1} K_{2}^{2} p} \sum_{n=0}^{\infty} 4 n e^{-2 n q_{2} l_{2}}
\end{aligned}
$$

The inverse Laplace transform is taken term by term from standard tables:

$$
L^{-1}\left(\frac{l_{1} K_{1} q_{1}^{2} \operatorname{coth}^{2}\left(l_{2} q_{2}\right)}{K_{2}^{2} q_{2}^{2} p}\right)=\frac{l_{1} K_{1} \alpha_{2}}{\alpha_{1} K_{2}^{2}} \sum_{n=0}^{\infty} 4 n\left(1-\operatorname{Erf}\left(\frac{n_{2}}{\sqrt{\alpha_{2} t}}\right)\right)
$$

\section{Result}

$v_{0}$ is obtained by adding the results of the inversions:

$$
\begin{aligned}
& \mathrm{v}_{0}=\mathrm{F}_{0}\left(\frac{1_{1}}{\mathrm{~K}_{1}}+\frac{2 \sqrt{\alpha_{2} \mathrm{t}}}{\mathrm{K}_{2}}\left(\sum_{n=0}^{\infty}\left(\operatorname{ierfc}\left(\frac{\mathrm{nl}}{\sqrt{\alpha_{2} \mathrm{t}}}\right)+\operatorname{ierfc}\left(\frac{(\mathrm{n}+1) \mathrm{l}_{2}}{\sqrt{\alpha_{2} \mathrm{t}}}\right)\right)\right)\right. \\
& \left.-\frac{l_{1}^{2} \alpha_{2}}{\alpha_{1} K_{2} I_{2}}\left(1+2 \sum_{n=1}^{\infty} e^{-\frac{n^{2} \pi^{2} \alpha_{2} l}{l_{2}^{2}}}\right)-\frac{l_{1} K_{1} \alpha_{2}}{\alpha_{1} K_{2}^{2}} \sum_{n=0}^{\infty} 4 n\left(1-\operatorname{Erf}\left(\frac{n l_{2}}{\sqrt{\alpha_{2} t}}\right)\right)\right)
\end{aligned}
$$




\section{APPENDIX C}

\section{DETAILED DERIVATIONS FOR THREE LAYER MODEL}

\section{A Notations}

In the following discussions, the multilayer structure is represented by a one dimensional model. Layer $n$ has thickness $I_{n}$, thermal diffusivity $\alpha_{n}$, thermal conductivity $K_{n}$ and is in perfect thermal contact with adjacent layers. Convection losses at the first and last layers are neglected. $t$ is the time, $p$ the Laplace variable and $q_{n}=\sqrt{p / \alpha_{n}}$.

\section{B Inversion of $\overline{\mathbf{v}}_{\mathbf{3}}$}

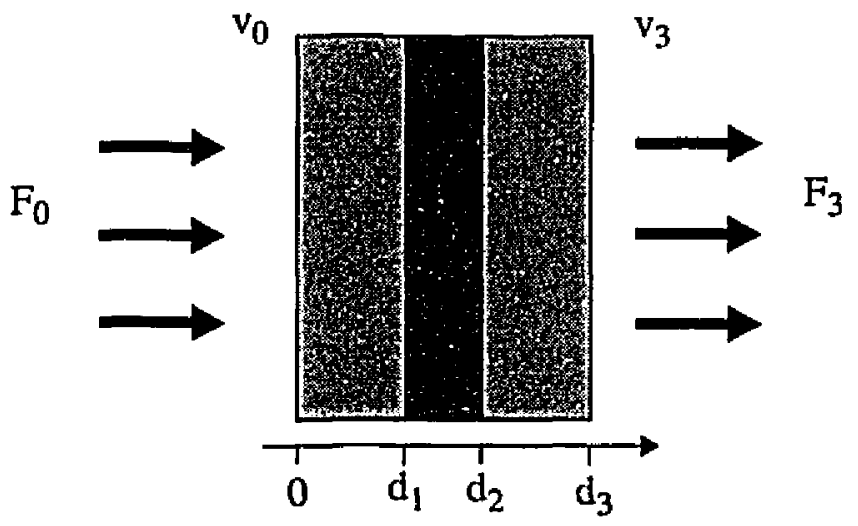

Fig. C.1 Model for a three layer structure.

For a three layer structure with the first and last layers identical, the full form for the 
transfer matrix $\left[\begin{array}{ll}A & B \\ C & D\end{array}\right]$ is:

$A=\left(K_{1} q_{1} \sinh \left(l_{1} q_{1}\right)\left(\frac{\cosh \left(l_{2} q_{2}\right) \sinh \left(l_{1} q_{1}\right)}{K_{1} q_{1}}+\frac{\cosh \left(l_{1} q_{1}\right) \sinh \left(l_{2} q_{2}\right)}{K_{2} q_{2}}\right)\right.$

$\left.+\cosh \left(l_{1} q_{1}\right)\left(\cosh \left(l_{1} q_{1}\right) \cosh \left(l_{2} q_{2}\right)+\frac{K_{2} q_{2} \sinh \left(l_{1} q_{1}\right) \sinh \left(l_{2} q_{2}\right)}{K_{1} q_{1}}\right)\right)$

$B=-\left(\cosh \left(l_{1} q_{1}\right)\left(\frac{\cosh \left(l_{2} q_{2}\right) \sinh \left(l_{1} q_{1}\right)}{K_{1} q_{1}}+\frac{\cosh \left(l_{1} q_{1}\right) \sinh \left(l_{2} q_{2}\right)}{K_{2} q_{2}}\right)\right.$ $\left.+\frac{\sinh \left(l_{1} q_{1}\right)\left(\cosh \left(l_{1} q_{1}\right) \cosh \left(l_{2} q_{2}\right)+\frac{K_{2} q_{2} \sinh \left(l_{1} q_{1}\right) \sinh \left(l_{2} q_{2}\right)}{K_{1} q_{1}}\right)}{K_{1} q_{1}}\right)$

$C=-\left(\cosh \left(l_{1} q_{1}\right)\left(K_{1} q_{1} \cosh \left(l_{2} q_{2}\right) \sinh \left(l_{1} q_{1}\right)+K_{2} q_{2} \cosh \left(l_{1} q_{1}\right) \sinh \left(l_{2} q_{2}\right)\right)\right.$

$\left.+K_{1} q_{1} \sinh \left(l_{1} q_{1}\right)\left(\cosh \left(l_{1} q_{1}\right) \cosh \left(l_{2} q_{2}\right)+\frac{K_{1} q_{1} \sinh \left(l_{1} q_{1}\right) \sinh \left(l_{2} q_{2}\right)}{K_{2} q_{2}}\right)\right)$

$D=\frac{\sinh \left(l_{1} q_{1}\right)\left(K_{1} q_{1} \cosh \left(l_{2} q_{2}\right) \sinh \left(l_{1} q_{1}\right)+K_{2} q_{2} \cosh \left(l_{1} q_{1}\right) \sinh \left(l_{2} q_{2}\right)\right)}{K_{1} q_{1}}$

$+\cosh \left(l_{1} q_{1}\right)\left(\cosh \left(l_{1} q_{1}\right) \cosh \left(l_{2} q_{2}\right)+\frac{K_{1} q_{1} \sinh \left(l_{1} q_{1}\right) \sinh \left(l_{2} q_{2}\right)}{K_{2} q_{2}}\right)$

where $\mathrm{l}_{2}=\mathrm{d}_{2}-\mathrm{d}_{1}$ and $\mathrm{l}_{1}=\mathrm{d}_{1}-0=\mathrm{d}_{3}-\mathrm{d}_{2}$. 
The Laplace transform of the temperature due to a short heat pulse with amplitude $F_{0}$ at $t=0$ with no flux at the back surface is evaluated by taking the ratio $B-(A D) / C$ :

$$
\begin{aligned}
& \bar{v}_{3}=\left(4 \mathrm{~F}_{0} \mathrm{~K}_{2} / \sqrt{\mathrm{p} \alpha_{2}}\right) /\left(2\left(\frac{\mathrm{K}_{2}^{2}}{\alpha_{2}}-\frac{\mathrm{K}_{1}^{2}}{\alpha_{1}}\right) \sinh \left(\mathrm{I}_{2} \sqrt{\frac{\mathrm{p}}{\alpha_{2}}}\right)\right. \\
& -\left(\frac{\mathrm{K}_{1}}{\sqrt{\alpha_{1}}}-\frac{\mathrm{K}_{2}}{\sqrt{\alpha_{2}}}\right)^{2} \sinh \left(\left(\frac{2 \mathrm{I}_{1}}{\sqrt{\alpha_{1}}}-\frac{\mathrm{l}_{2}}{\sqrt{\alpha_{2}}}\right) \sqrt{\mathrm{p}}\right) \\
& \left.+\left(\frac{\mathrm{K}_{1}}{\sqrt{\alpha_{1}}}+\frac{\mathrm{K}_{2}}{\sqrt{\alpha_{2}}}\right)^{2} \sinh \left(\left(\frac{21_{1}}{\sqrt{\alpha_{1}}}+\frac{\mathrm{l}_{2}}{\sqrt{\alpha_{2}}}\right) \sqrt{\mathrm{p}}\right)\right)
\end{aligned}
$$

In order to simplify the expression [C.2], reduced parameters are used:

$$
\mathrm{L}_{1}=\frac{2 \mathrm{l}_{1}}{\sqrt{\alpha_{1}}} \quad \mathrm{~L}_{2}=\frac{\mathrm{l}_{2}}{\sqrt{\alpha_{2}}} \quad \mathrm{k}_{1}=\frac{\mathrm{K}_{1}}{\sqrt{\alpha_{1}}} \quad \mathrm{k}_{2}=\frac{\mathrm{K}_{2}}{\sqrt{\alpha_{2}}}
$$

By replacing the hyperbolic functions by their exponential definitions and using the simplifications of Eq. (3), $\bar{v}_{3}$ becomes:

$$
\begin{aligned}
& \overline{v_{3}}=\left(\frac{8 F_{0} k_{2}}{\sqrt{p}}\right)\left(\left(2\left(k_{2}^{2}-k_{1}^{2}\right)\left(e^{L_{2} \sqrt{p}}-e^{-L_{2} \sqrt{p}}\right)+\left(k_{1}-k_{2}\right)^{2}\left(e^{-\left(L_{1}-L_{2}\right) \sqrt{p}}-e^{\left(L_{1}-L_{2}\right) \sqrt{p}}\right)\right.\right. \\
& \left.+\left(k_{1}+k_{2}\right)^{2}\left(e^{\left(L_{1}+L_{2}\right) \sqrt{p}}-e^{-\left(L_{1}+L_{2}\right) \sqrt{p}}\right)\right)
\end{aligned}
$$

Multiplying numerator and denominator by $\mathrm{e}^{-L_{2} \sqrt{\mathfrak{p}}}$ :

$$
\begin{aligned}
& \bar{v}_{3}=\left(\frac{8 F_{0} k_{2} e^{-L_{2} \sqrt{p}}}{\sqrt{p}}\right)\left(2\left(k_{2}^{2}-k_{1}^{2}\right)\left(1-e^{-2 L_{2} \sqrt{p}}\right)+\left(k_{1}-k_{2}\right)^{2}\left(e^{-L_{1} \sqrt{p}}-e^{\left(L_{1}-2 L_{2}\right) \sqrt{p}}\right)\right. \\
& \left.+\left(k_{1}+k_{2}\right)^{2}\left(e^{L_{1} \sqrt{p}}-e^{-\left(L_{1}+2 L_{2}\right) \sqrt{p}}\right)\right)
\end{aligned}
$$


Rearranging the denominator with powers of $\mathrm{e}^{-\mathrm{L}_{2} \sqrt{\mathrm{p}}}$ :

$$
\begin{aligned}
& \overline{v_{3}}=\left(\frac{8 F_{0} k_{2} e^{-L_{2} \sqrt{p}}}{\sqrt{p}}\right) /\left(\left(\left(k_{1}-k_{2}\right)^{2} e^{-L_{1} \sqrt{p}}+\left(k_{1}+k_{2}\right)^{2} e^{L_{1} \sqrt{p}}+2\left(k_{2}^{2}-k_{1}^{2}\right)\right)\right. \\
& \left.-\left(\left(k_{1}-k_{2}\right)^{2} e^{L_{1} \sqrt{p}}+\left(k_{1}+k_{2}\right)^{2} e^{-L_{1} \sqrt{p}}+2\left(k_{2}^{2}-k_{1}^{2}\right)\right) e^{-2 L_{2} \sqrt{p}}\right)
\end{aligned}
$$

The denominator can be expanded into an infinite series :

$$
\bar{v}_{3}=\left(\frac{8 F_{0} k_{2}}{\sqrt{p}}\right) \sum_{n=0}^{\infty} \frac{\left(\left(k_{1}-k_{2}\right)^{2} e^{L_{1} \sqrt{p}}+\left(k_{1}+k_{2}\right)^{2} e^{-L_{1} \sqrt{p}}+2\left(k_{2}^{2}-k_{1}^{2}\right)\right)^{n} e^{-(2 n+1) L_{2} \sqrt{p}}}{\left(\left(k_{1}-k_{2}\right)^{2} e^{-L_{1} \sqrt{p}}+\left(k_{1}+k_{2}\right)^{2} e^{L_{1} \sqrt{p}}+2\left(k_{2}^{2}-k_{1}^{2}\right)\right)^{n+1}}
$$

\section{Rearranging :}

$$
\overline{v_{3}}=
$$

$$
\left(\frac{8 F_{0} k_{2}}{\sqrt{p}}\right) \sum_{n=0}^{\infty}\left(\frac{e^{-(2 n+1) L_{2} \sqrt{p}}\left(\left(k_{1}-k_{2}\right)^{2}+\left(k_{1}+k_{2}\right)^{2} e^{-2 L_{1} \sqrt{p}}+2\left(k_{2}^{2}-k_{1}^{2}\right) e^{-L_{1} \sqrt{p}}\right)^{n} e^{n L_{1} \sqrt{p}}}{\left(\left(k_{1}-k_{2}\right)^{2} e^{-2 L_{1} \sqrt{p}}+\left(k_{1}+k_{2}\right)^{2}+2\left(k_{2}^{2}-k_{1}^{2}\right) e^{L_{1} \sqrt{p}}\right)^{n+1} e^{(n+1) L_{1} \sqrt{p}}}\right)
$$

Expression in Eq. (8) can be expanded:

$$
\begin{aligned}
& \overrightarrow{v_{3}}=\left(\frac{8 F_{0} k_{2}}{\sqrt{p}}\right)\left(\sum_{n=0}^{\infty}\left(e^{-\left(L_{1}+(2 n+1) L_{2}\right) \sqrt{p}}\right) \sum_{m=0 i=0}^{\infty} \sum_{j=0}^{m}\left(\left(\sum_{j=}^{m-i} \Psi_{1}\right)\left(\sum_{s=0}^{i} \Psi_{2}\right)\right) e^{-m L_{1} \sqrt{p}}\right)(9) \\
& \text { with } \quad \Psi_{1}=\left(\begin{array}{c}
n \\
j
\end{array}\right)\left(\begin{array}{c}
j \\
m-j-i
\end{array}\right)\left(k_{1}+k_{2}\right)^{2(m-i-j)}\left(k_{1}-k_{2}\right)^{2(n-j)}\left(2\left(k_{1}^{2}-k_{2}^{2}\right)\right)^{i+2 j-m} \\
& \text { and } \quad \Psi_{2}=\left(\begin{array}{c}
-1-n \\
s
\end{array}\right)\left(\begin{array}{c}
s \\
i-s
\end{array}\right)\left(k_{1}+k_{2}\right)^{2(-1-n-s)}\left(k_{1}-k_{2}\right)^{2(i-s)}\left(2\left(k_{1}^{2}-k_{2}^{2}\right)\right)^{-i+2 s}
\end{aligned}
$$


Finally, the expression to be inverted is:

$$
\overrightarrow{v_{3}}=\left(\frac{8 F_{0} k_{2}}{\sqrt{p}}\right) \sum_{n=0}^{\infty} \sum_{m=0}^{\infty}\left(\sum_{i=0}^{m}\left(\left(\sum_{j=0}^{m-i} \Psi_{1}\right)\left(\sum_{s=0}^{i} \Psi_{2}\right)\right) e^{-\left((m+1) L_{i}+(2 n+1) L_{2}\right) \sqrt{p}}\right)
$$

The solution can be obtained from standard tables of transforms. With $\Psi_{1}$ and $\Psi_{2}$ defined in Eq. (9), v3 is:

$$
v_{3}=\frac{8 F_{0} k_{2}}{\sqrt{\pi t}}\left(\sum_{n=0}^{\infty} \sum_{m=0}^{\infty}\left(\sum_{i=0}^{m}\left(\sum_{j=0}^{m-i} \Psi_{1}\right)\left(\sum_{s=0}^{i} \Psi_{2}\right)\right) e^{\left.-\frac{\left((2 n+1) L_{2}+(m+1) L_{1}\right)^{2}}{4 t}\right)}\right.
$$




\title{
APPENDIX D \\ DIFFUSIVITY MEASUREMENT USING QUASI PERIODIC TEMPERATURE RESPONSE MODELING
}

\author{
Pierre R. Emeric \\ Applied Science Program \\ The College of William \& Mary in Virginia \\ Williamsburg, VA 23187-8795 \\ William P. Winfree \\ MS 231 NASA Langley Research Center \\ Hampton, VA 23681-0001
}

\section{INTRODUCTION}

Periodic heat flow methods for measurement of the diffusivity of a material have proven to be highly effective methods dating back to the work of Angstrom. Typically, these measurements have involved a determination of the phase lag between a source applied to the surface and the temperature measured at the opposite surface. When the frequency of the periodic heating is well matched to specimen, it represents perhaps the most efficient thermal diffusivity measurement technique.

For accurate phase measurement, frequency components from the transients from the start of the heat application and long term variations such as sample heat up must be filtered 
out. For a point detector, this is simply done by passing the output of the detector through a band pass filter. For a scanned detector, where the desire is to measure the spacial variations in the diffusivity, it is difficult to pass the output of the scanned sensor through an analog band pass filter. One approach is to digitize a long time record and post process the data to remove relevant frequency components. This is undesirable because it requires a large amount of real time data storage, which is often expensive. A second technique is to allow the specimen to come to thermal equilibrium with the environment. This is practical for thin samples with high diffusivities where the frequency is high. However, for many structures, the frequencies required for heat to be able to penetrate the structure is on the order of 1 or less Hertz. The time required for the structure to come to equilibrium may be on the order of several minutes.

To remove some of the difficulties, the thermal diffusivity can be determined from analytical solution which incorporates the transient terms. This enables an effective use of the memory required for storage of the images while still taking advantage of the periodic heating. It also allows the measurements to be performed in a shorter time period.

The analytical solution is used in a non linear least square routine to estimate the diffusivity of several samples divided by their thickness squared. The technique is applied to samples with varying thicknesses and diffusivity. A comparison of the results from these measurements and other measurements is presented. 


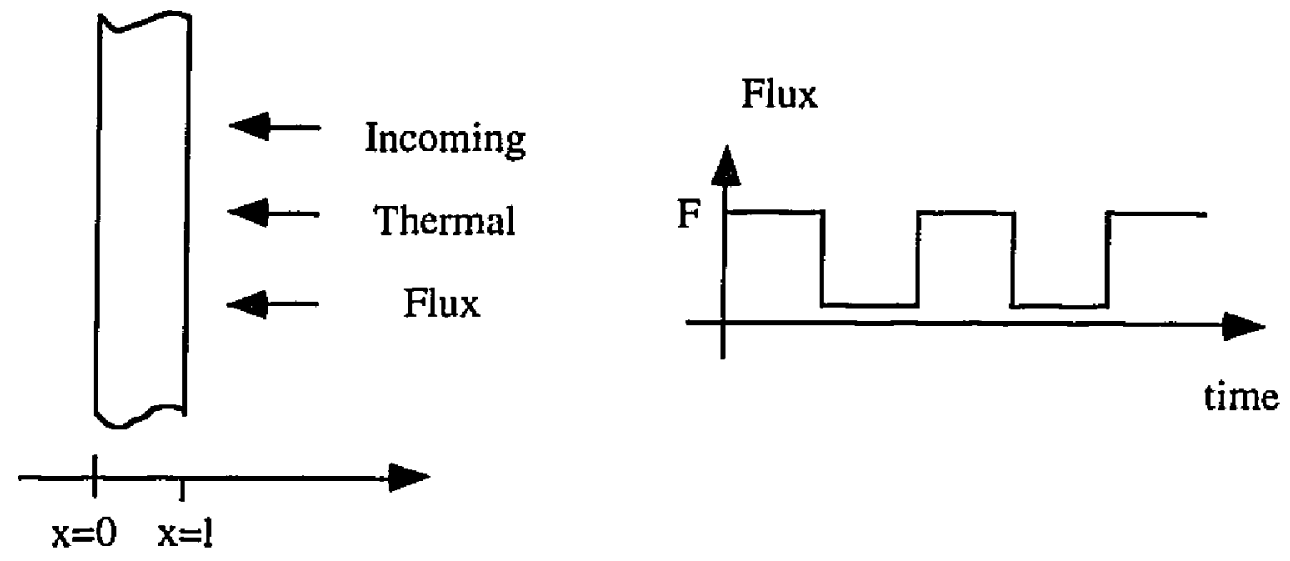

Fig. 1 Slab with incoming square pulse heat flux at the back surface

\section{THERMAL RESPONSE OF THIN PLATES}

The thermal response of the slab is modeled by assuming that it is a single homogeneous layer undergoing a square pulse irradiation on its back face (Fig. 1). Convection losses are neglected. For a flux input that is evenly applied over the surface of the sample and a thin plate structure where the lateral variations are small relative to its thickness, a one dimensional solution is appropriate.

As described in Fig 1, the front surface is at $\mathrm{x}=0$ and the back end at $\mathrm{x}=\mathrm{l}$. The 1-dimensional heat equation and the boundary conditions can be written down as : 


$$
\frac{\partial^{2} \theta}{\partial x^{2}}=\frac{1}{\alpha} \frac{\partial \theta}{\partial t} \quad \text { for } 0 \leq x \leq 1
$$

where $\theta$ is the temperature, $\alpha$ the thermal diffusivity, $t$ the elapsed time after the beginning of the experiment and $x$ the horizontal coordinate.

$$
\text { at } \begin{aligned}
x=1, \quad k \frac{\partial \theta}{\partial x} & =F & & \text { for } 2 n \tau<t<(2 n+1) \tau \\
& =0 & & \text { otherwise. }
\end{aligned}
$$

where $\mathrm{k}$ is the thermal conductivity, $\mathrm{F}$ the amplitude of the incoming flux, $\tau$ the length of the heat pulse and $\mathrm{n}$ the number of pulses.

$$
\text { at } x=0, \quad k \frac{\partial \theta}{\partial x}=0
$$

$$
\text { at } t=0, \theta(x, 0)=0 \quad \text { (the initial temperature is set at zero ). }
$$

A periodic square pulse signal can be represented as a sum of step functions shifted in time and changed in sign. The solution is found by superposing the solutions to the simpler problems with the incoming heat flux profile described as a step function (Ref. 1). The solutions are :

$$
\theta_{\text {step function }}(t)=0 \text { for } t \leq 0
$$

$\theta_{\text {step function }}(t)=\frac{2 F \sqrt{\alpha t}}{k} \sum_{n=0}^{\infty} 2 \operatorname{ierfc}\left(\frac{(2 n+1) 1}{2 \sqrt{\alpha t}}\right) \quad$ for $0 \leq t \leq \frac{\left.3.5\right|^{2}}{\alpha \pi^{2}}$ 
$\theta_{\text {step function }}(t)=\frac{F t}{\rho c l}+\frac{F l}{k}\left(\frac{1}{6}-\frac{2}{\pi^{2}} \sum_{n=1}^{\infty} \frac{(-1)^{n}}{n^{2}} e^{\left(\frac{-\alpha \ln 2 \pi^{2}}{1^{2}}\right)}\right)$ for $t \geq \frac{3.5 l^{2}}{\alpha \pi^{2}}$

where $\rho$ and $c$ are the density and specific heat of the material.

Thus, $\quad \theta(t)=\sum_{n=0}^{2 N-1}(-1)^{n} \theta_{\text {step function }}(t-n \tau)$

where $\mathrm{N}$ is the number of cycles, is the solution to the original problem.

As can be seen from equations [5] and [6], the diffusivity is most directly obtainable from the thermal response, rather, the thermal response depends on $\alpha / l^{2}$. If two parameters are varied, such as $\alpha / 1^{2}$ and $F / p c l$, the thermal response as represented in equation [7] can be varied to minimize the difference between $\theta(t)$ and the measured thermal response. The criterion for minimization chosen is the minimum of the square difference between $\theta(t)$ and the measured response. This gives an estimate for the parameter $\alpha / l^{2}$. If $\alpha$ is known, $l$ can then be calculated. If $l$ is known, it is possible to find $\alpha$. 


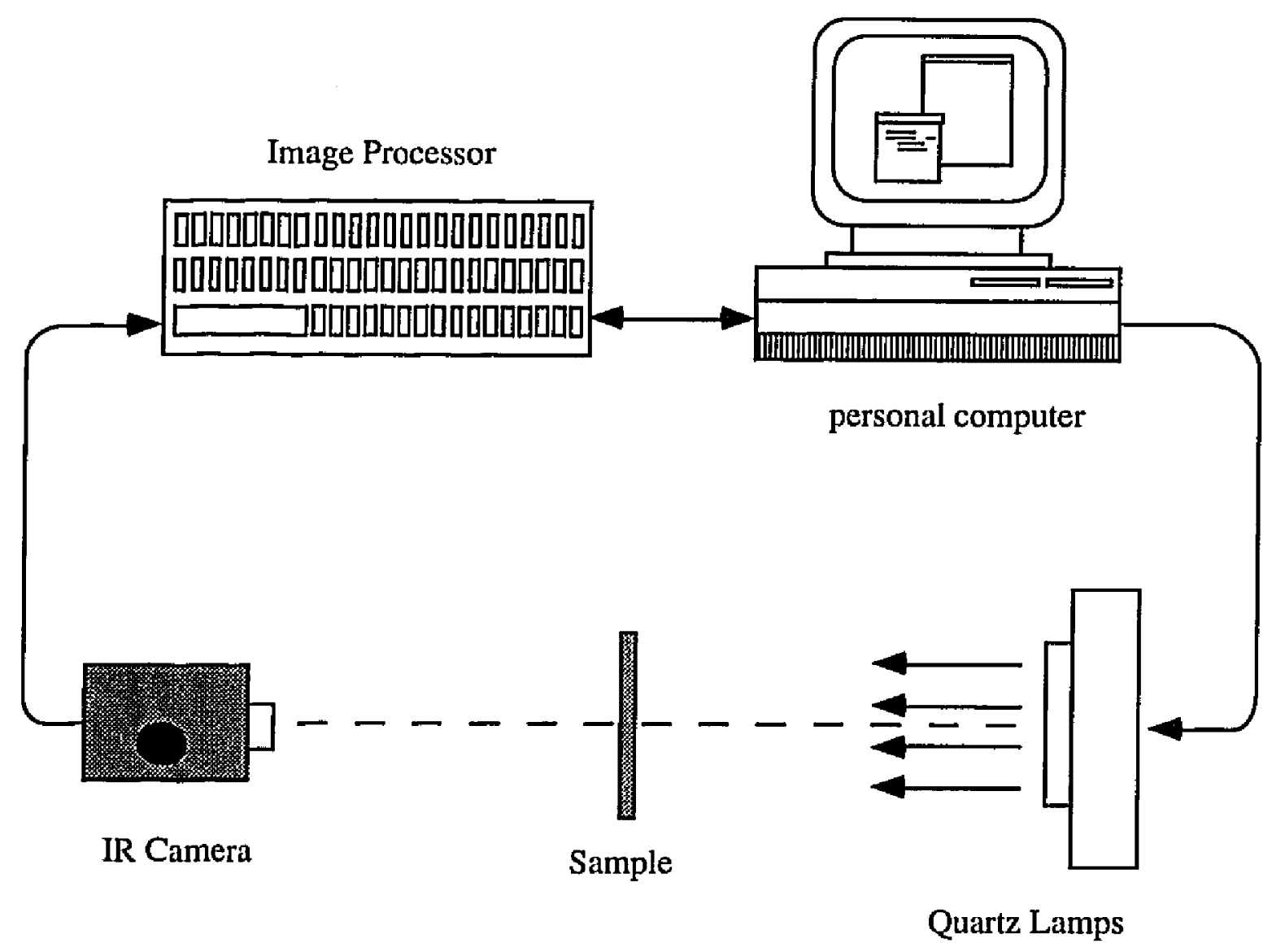

Fig. 2 Experimental setup for data acquisition.

\section{THERMAL DIFFUSIVITY MEASUREMENT SYSTEM}

A block diagram of the experiment setup is shown in Fig. 2. A commercial infrared camera radiometrically measured the change in surface temperature as a function of time. The output of the infrared camera was digitized by an image processor. The image processor averaged the digitized images and stored the data in memory. The thermal stimuli was provided by 4 quartz tubes. The application of heat was synchronized with the digitization of the infrared image by a computer controller. The computer controlled the 
data acquisition by keeping a given phase of the period heating averaging into a fixed portion of memory. This minimized the amount of memory required, while maintaining a high temporal resolution.

\section{RESULTS}

To demonstrate the technique, measurements were performed on an aluminium sample. The sample geometry is shown in Fig. 3 . Three square holes were milled out at the back surface of the plate to vary the thickness as a function of position. They are, from left to right, $5.715 \mathrm{~mm}, 4.445 \mathrm{~mm}$ and $3.175 \mathrm{~mm}$ deep. A compilation of the measurements at different frequencies is given in table 1 . The frequency corresponds to $1 / 2 \tau$. The measurements represent the diffusivity as measured for the regions where no holes were present. The average value of diffusivity computed from the experiments fits the literature values for aluminium within a $7 \%$ accuracy.

From the data, an image, shown in Fig. 4 , was obtained for $\alpha / /^{2}$ for the sample. Since, for this case, the diffusivity of the sample is constant, the image corresponds to $1 / 1^{2}$. For comparison, the image directly obtained from the IR imager is also shown in the figure. As can be seen, the processed image enables detection of even the shallowest hole which is not visible in the infrared image. 


\begin{tabular}{|l||ll|}
\hline Frequency $(\mathrm{Hz})$ & \multicolumn{2}{|c|}{ Diffusivity $\left(\frac{\mathrm{m}^{2}}{\mathrm{~s}}\right)$} \\
\hline 1.07 & 97.0 & $10^{-6}$ \\
1.60 & 96.1 & $10^{-6}$ \\
2.13 & 78.5 & $10^{-6}$ \\
& & \\
\hline
\end{tabular}

Table 1. Measurements for different frequencies. The average measured value for diffusivity is $90.510^{-6} \mathrm{~m}^{2} / \mathrm{s}$. Literature value for Aluminium averages $97.510^{-6} \mathrm{~m}^{2} / \mathrm{s}$.

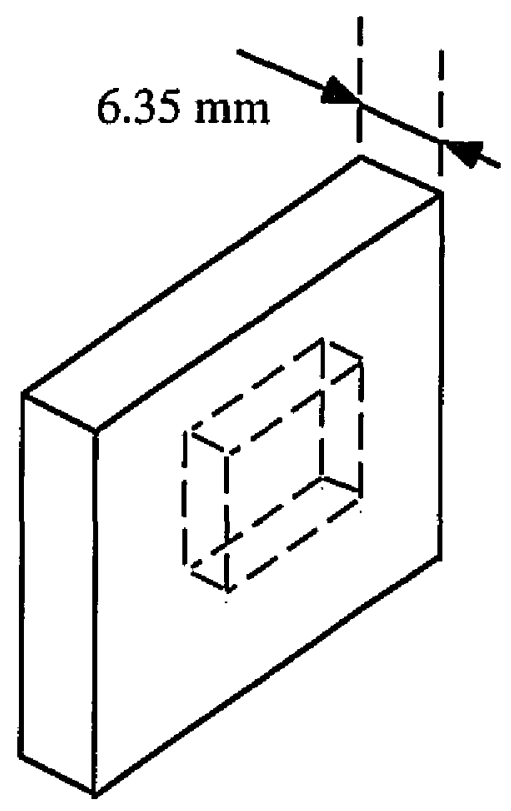

Fig. 3 Geometry of a portion of the Aluminium sample. 


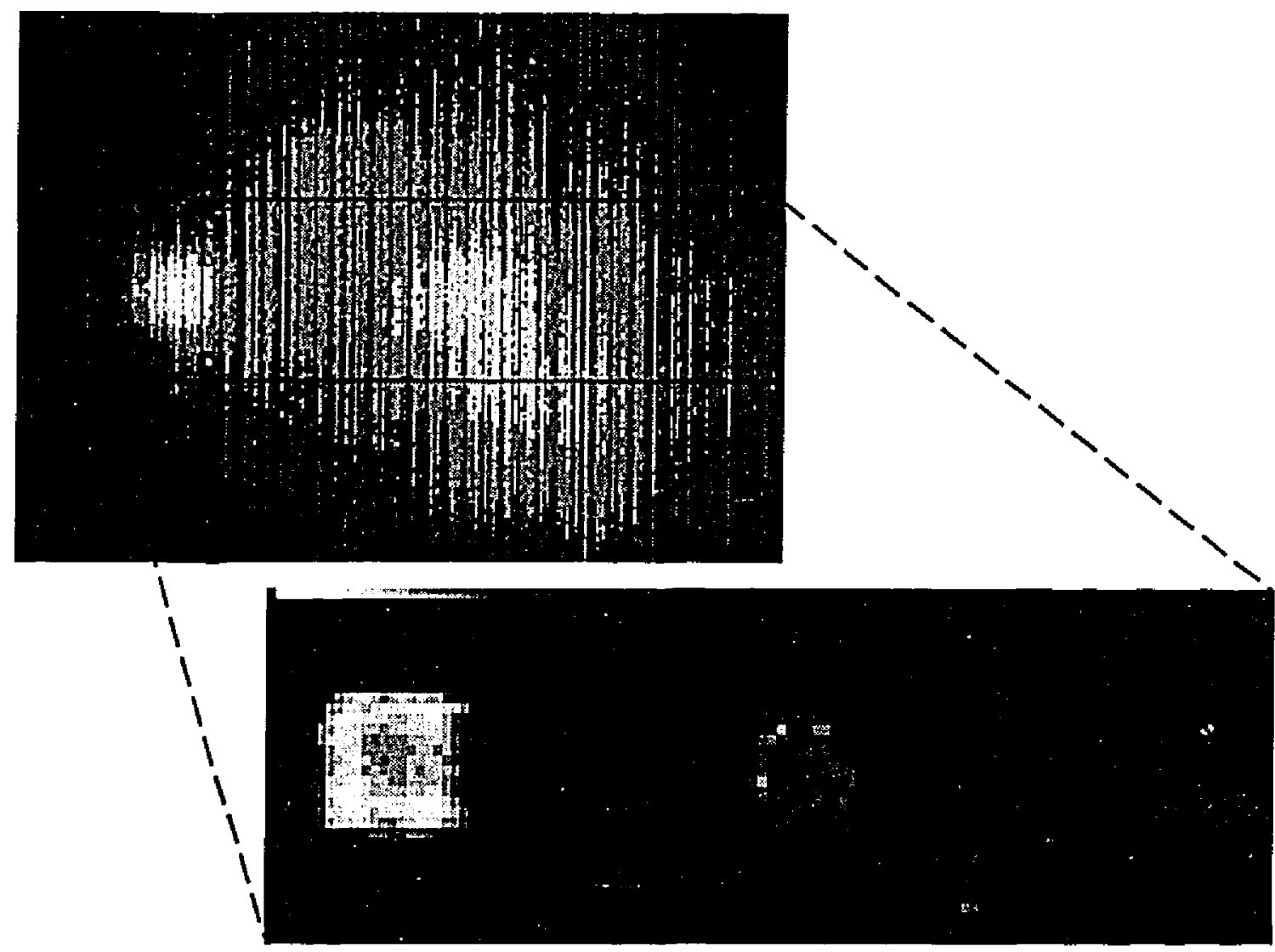

$5.715 \mathrm{~mm}$

$4.445 \mathrm{~mm}$

$3.175 \mathrm{~mm}$

Top view

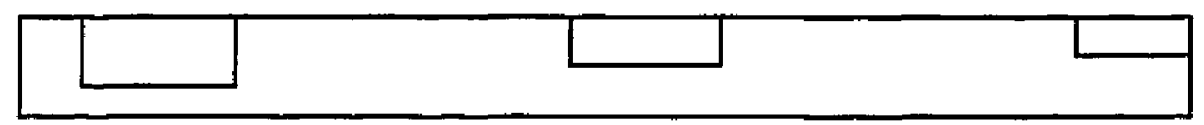

Fig. 4 Diffusivity image obtained from the thermograms of the Aluminium sample.

Measurements were also made on graphite epoxy composite samples with varying fiber volume fraction. The samples were a set of 6 simple panels (16 plies : [0/90] $4 \mathrm{~s}$ ) with fiber volume fraction $40 \%, 50 \%, 60 \%, 65 \%, 70 \%$ and $74 \%$. The results obtained using the quasiperiodic method are shown in Fig. 5. Also shown are the measurements made using Angstrom's method (Ref. 2). Both curves indicate that the diffusivity increases with the fiber volume fraction. The difference in absolute value is assumed to be 
due to no convection loss correction in either measurements. The high value of porosity in the samples where the fiber volume fraction is above $65 \%$ is an explanation for the plateau. Fig. 6 shows the diffusivity images obtained from the samples. The increase in spacial variation in the diffusivity images for a fiber volume fraction of $65 \%$ and above may correspond to the high porosity.

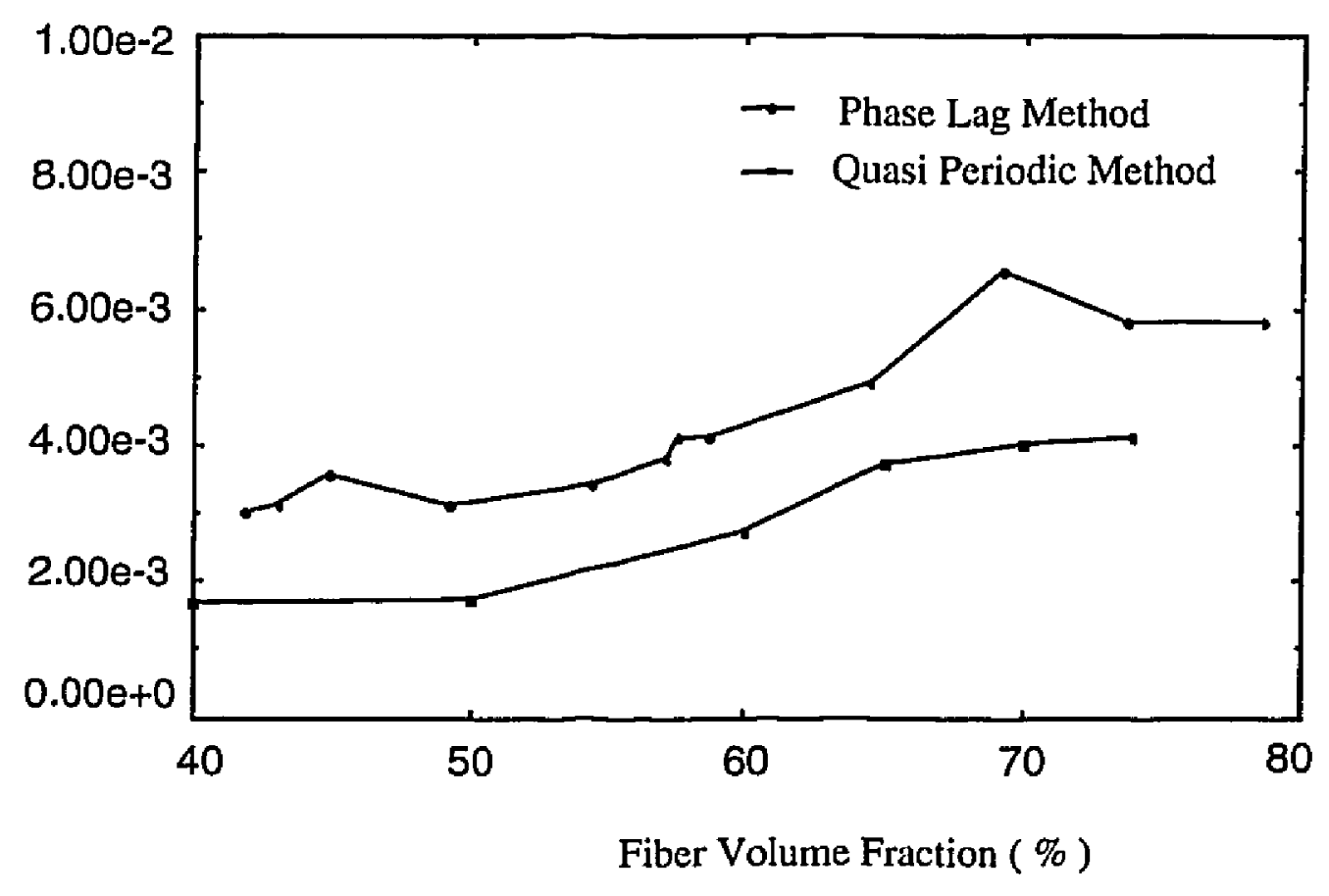

Fig. 5 Diffusivity measurements on Graphite Epoxy Composite samples as a function of the fiber volume fraction. Top curve corresponds to Angstrom method, bottom curve to Quasi periodic method. The values of diffusivity are given in $\mathrm{cm}^{2} / \mathrm{s}$ 

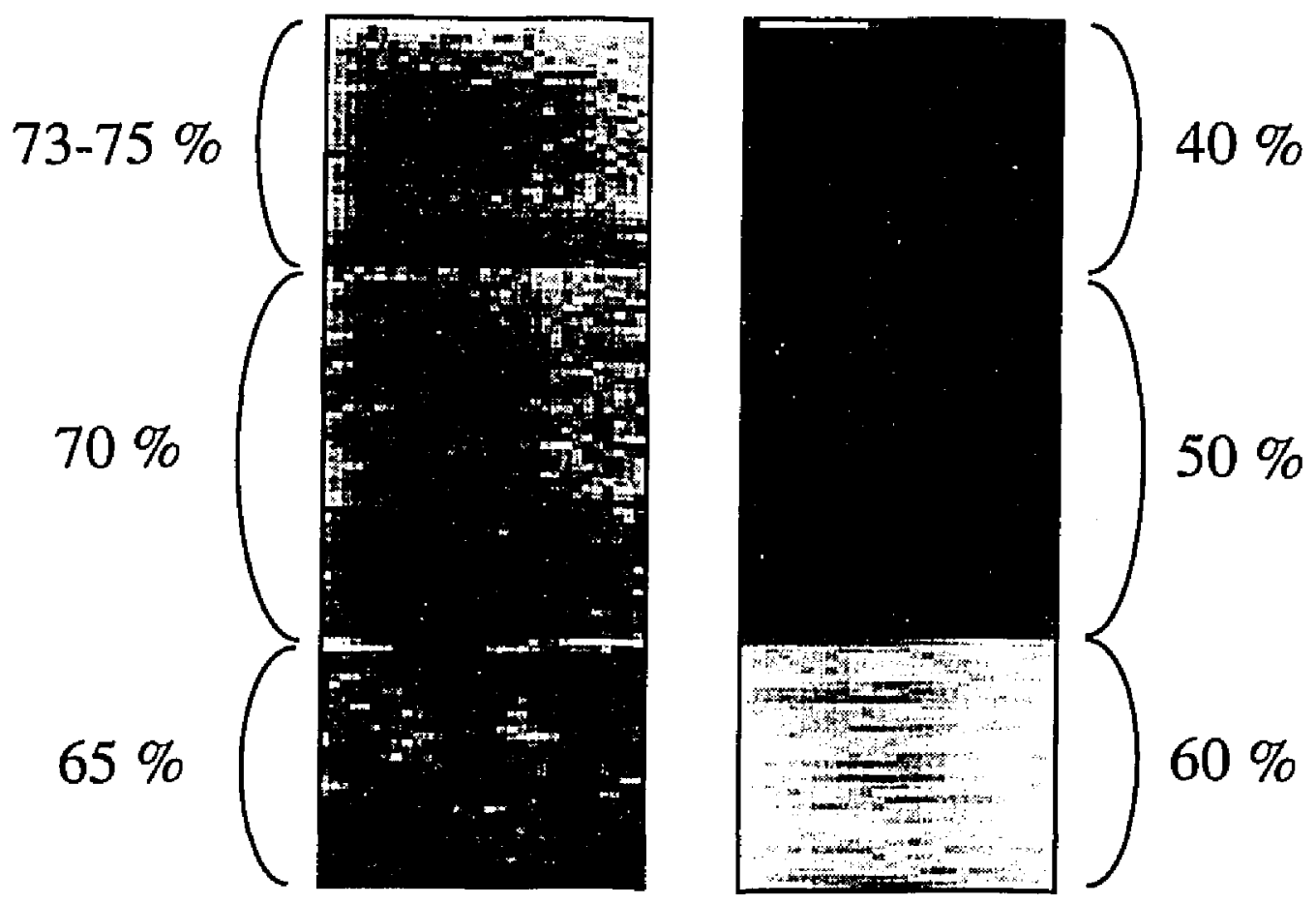

Fig.6 Diffusivity images of Graphite Epoxy Composite samples. The grey scale look up tables used in the two images are linear but different. Due to the small differences in diffusivity values for the last three samples ( $65 \%, 70 \%, 74 \%$ in fiber volume fraction ) the contrast has been linearly stretched for a better visualization.

Finally, diffusivity measurements using the quasi periodic method were made on a Carbon-Carbon composite panel with four stiffeners at the back surface. Fig. 7 shows the geometry of the stiffeners and indicates the thicknesses.

As in Fig. 4 , the image in Fig.8 shows the variations in thicknesses of the CarbonCarbon panel. The background grey level corresponds to a measured average diffusivity value of $0.119 \mathrm{~cm}^{\wedge} 2 / \mathrm{s}$. This demonstrates the ability of the technique to characterize complex geometries. 


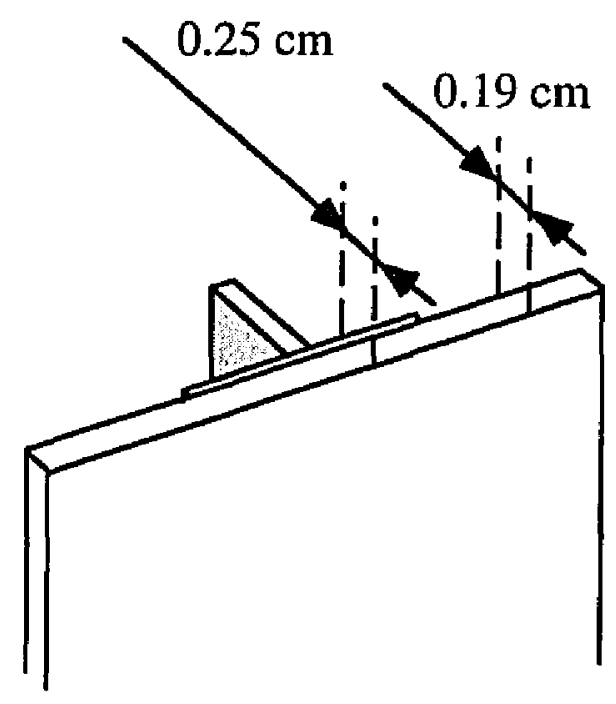

Fig.7 Detail of a stiffener on the Carbon-Carbon panel.

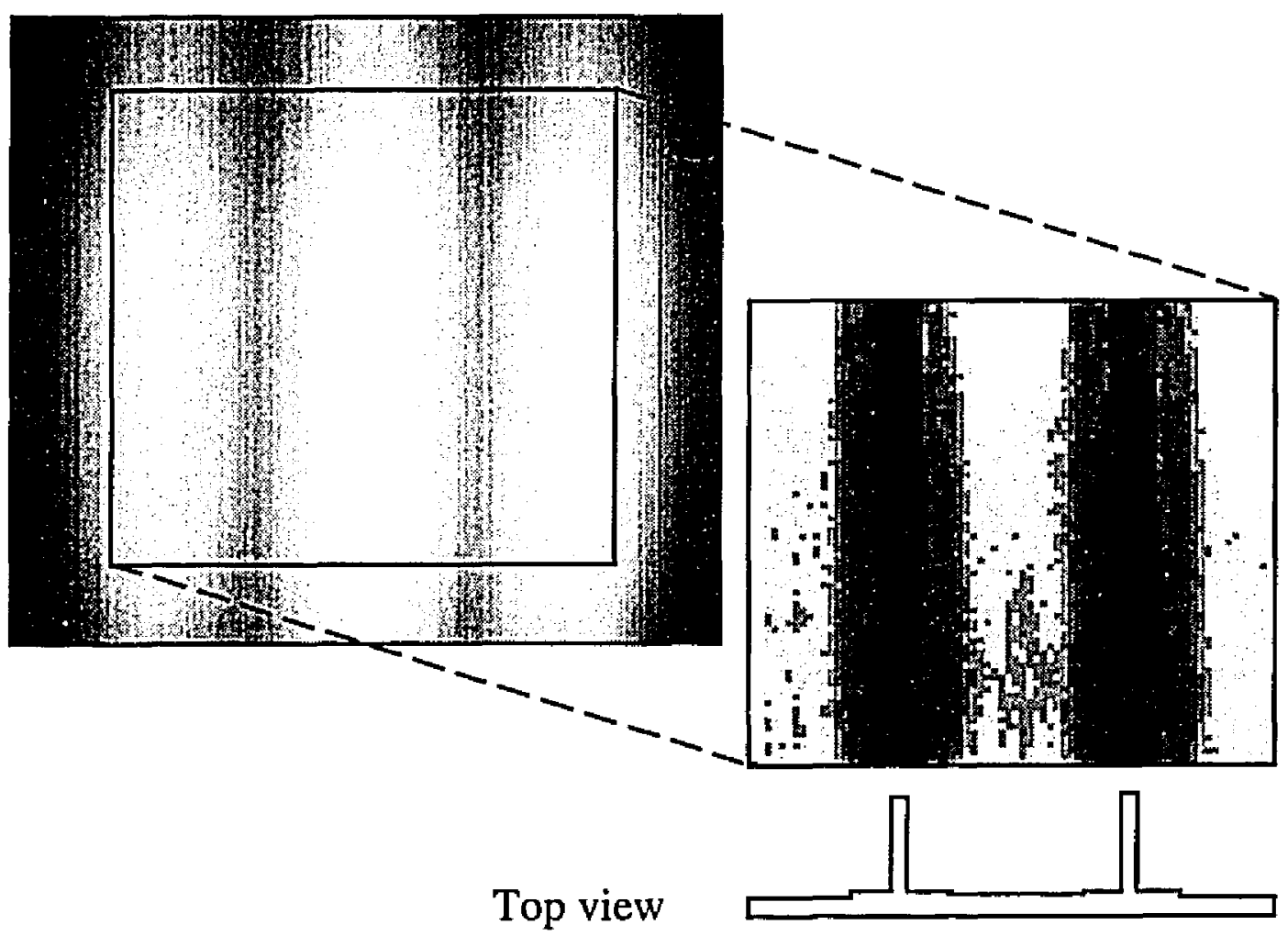

Fig. 8 Thermogram and diffusivity image of the Carbon-Carbon composite panel. 


\section{CONCLUSIONS}

A model for through-the-thickness diffusivity imaging was applied to Aluminium and composite materials. This model, by taking into account the transient terms in the temperature response, allows quick measurements. The results obtained using the quasi periodic method are found in good agreement with either the literature values or with the diffusivity values obtained by other experimental methods.

\section{REFERENCES}

1. H. S. Carslaw and J. C. Jaeger, Conduction of Heat in Solids, 2nd edition, Oxford Science Publications, p. 112 .

2. Quantitative Thermal Diffusivity Measurements on Composite Fiber Volume Fraction Samples, by Joe Zalameda and William P. Winfree, NASA Langley Research Center, MS 231, Hampton, VA 23681-0001, presented at QNDE 92, La Jolla, Ca. 


\section{NOTES}

1. H. S. Carslaw \& J. C. Jaeger, Conduction of heat in solids, 2nd edition, Clarendon Press, Oxford (1959), p. 136.

2. J.H. Becker, Several new methods to measure the thermal diffusivity of semi-conductors, Journal of Applied Physics, Vol. 31, No. 3, pp. 612-613, March 1960.

3. A. Hirschman, J. Dennis, W. Derksen, T. monahan, An optical method for measuring the thermal diffusivity of solids, International developments in heat transfer, ASME, Part IV, pp. 863-869.

4. L. Qian, P. Li, Photothermal radiometry measurement of thermal diffusivity journal of Applied Optics, Vol. 29, No. 28, pp. 4241-4243, Oct 1990.

5. W. B. Jackson, N. M. Amer, A. C. Boccara and D. Fournier, Photothermal deflection spectroscopy and detection, Journal of Applied Optics, Vol. 20, No. 8, pp. 1333-1344, April 1981.

6. L. C. Aamodt and J. C. Murphy, Thermal measurements using a localized excitation source, Journal of Applied Physics, Vol. 52, No. 8, pp.4903-4914, Aug. 1981.

7. P. K. Kuo, E. D. Sendler, L. D. Favro and R. L. Thomas, Mirage-effect measurement of thermal diffusivity. Part II: theory, Canadian Journal of Physics, Vol. 64, No. 9, pp. 1168-1171, Sept. 1986.

8. W.J. Parker, R.J. Jenkins, C.P. Butler, G.L. Abbot, flash method of determining thermal diffusivity, heat capacity, and thermal conductivity, Journal of Applied Physics, Vol. 32, No. 9, pp. 1679-1684, Sept. 1961.

9. R.C. Heckman, Finite pulse-time and heat loss effects in pulse thermal diffusivity measurements, Journal of Applied Physics, Vol. 44, No. 4, pp. 1455-1460, April 1973.

10. S. K. Lau, D. P. Almond and J. M. Milne, A quantitative analysis of pulsed video thermography, NDT \& E International,Vol. 24, No. 4, pp. 195-202, Aug. 1991. 
11. D. P. Almond and S. K. Lau, Edge effects and a method of defect sizing for transient thermography, Applied Physics Letters 62 (25), pp. 3369-3371, 21 June 1993.

12. L.C. Aamodt, J.W. M. Spicer, J.C. Murphy, Analysis of characteristic thermal transit times for Time-Resolved Infrared Radiometry Studies of multilaered coatings, Journal of Applied Physics, Vol. 68, No. 12, Dec 1990.

13. J.W.M. Spicer, W.D. Kerns, L.C. Aamodt, R. Osiander, J.C. Murphy, Time-Resolved Infrared Radiometry (TRIR) using a focalplane Array for characterization of hiddencorrosion, SPIE Vol. 1933 Thermosense XV (1993)

14. J. C. Maxwell, A treatise on electricity and magnetism, $3^{\text {rd }}$ edition. Dover Publications, N.Y., 1954.

15. S. Nomura and A. Haji-Sheikh, Analysis of transient heat conduction in composites, Proceedings of the $3^{\text {rd }}$ Japan-US conference on composite materials, June 23-25, 1986. Tokyo, Japan Society for Composite Materials, 1986, pp. 821-828.

16. S. Nomura and T. Chou, Heat conduction in composite materials due to oscillating temperature field, International Journal of Engineering Science, Vol. 24, No. 5, 1986, pp. 643-647.

17. H. Hatta and M. Taya, Equivalent inclusion method for steady-state heat conduction in composites, International Journal of Engineering Science, Vol. 24, No. 7, 1986, pp. 1159-1172.

18. Y. Benveniste, Effective thermal conductivity of composites with a thermal contact resistance between the constituents: Nondilute case, Journal of Applied Physics, Vol. $61(8), 15$ April 1987.

19. D. P. H. Hasselman and L. F. Johnson, Effective thermal conductivity of composites with interfacial thermal barrier resistance, Journal of Composite materials, Vol. 21, June 1987.

20. D. P. H. Hasselman, H. Bhatt and K. Y. Donaldson, Role of the Interfacial Thermal Barrier in the effective Thermal Diffusivity/Conductivity of SiC-Fiber-Reinforced Reaction-Bonded.Silicon Nitride, J. Am. Ceram. Soc., Vol. 73 (2), pp. 312-316, 1990.

21. P. McCluskey, R. K. Williams, R. S. Graves and T. N. Tiegs, Thermal Diffussivity/ Conductivity of Alumina-Silicon Carbide composites, J. Am. Ceram. Soc., Vol. 73 (2), pp. 461-464, 1990.

22. H. S. Carslaw \& J. C. Jaeger, Conduction of heat in solids, 2nd edition, Clarendon Press, Oxford (1959), p. 326. 
23. H. S. Carslaw \& J. C. Jaeger, Conduction of heat in solids, 2nd edition, Clarendon Press, Oxford (1959), p. 263.

24. H. Stehfest, Numerical Inversion of Laplace Transforms, Communications of the ACM, Vol. 13, No. 1, pp. 47-49, Jan 1970.

25. P. M. Morse and H. Feshbach, Methods of theoretical physics, Part II, McGraw-Hill book company, Inc., 1953, p. 1372.

26. H. S. Carslaw \& J. C. Jaeger, Conduction of heat in solids, 2nd edition, Clarendon Press, Oxford (1959), p. 69.

27. Thermal diffusivity V. 10, IFI/Plenum - New York - Washington (1973), p. 518.

28. Thermal diffusivity V. 10, IFI/Plenum - New York - Washington (1973), p. 589.

29. C. Harper, Handbook of plastics and elastomers, McGraw-Hill (1975), p. 3-57.

30. CRC Handbook of Chemistry and Physics, $51^{\text {st }}$ edition, CRC Press (1970-1971), p. E4.

31. CRC Handbook of Chemistry and Physics, $51^{\text {st }}$ edition, CRC Press (1970-1971, p. E2. 


\section{BIBLIOGRAPHY}

H. S. Carslaw and J. C. Jaeger, Conduction of heat in solids, $2^{\text {nd }}$ edition, Oxford science publications, 1959.

M. Necati Özisik, Boundary value problems of heat conduction, International textbook company, 1968.

C. Kittel, Introduction to solid state physics, $4^{\text {th }}$ edition, John Wiley \& Sons, Inc.

P. M. Morse and H. Feshbach, Methods of theoretical physics, Part II, McGraw-Hill Book Company, Inc., 1953.

J. Mathews and R. L. Walker, Mathematical methods of physics, $2^{\text {nd }}$ edition, W. A. Benjamin, Inc., New York, 1970.

W. H. Press, B. P. Flannery, S.A. Teukolsky and W. T. Vetterling, Numerical recipes in C, Cambridge University Press, 1988.

S. Wolfram, Mathematica, a system for doing mathematics by computer, Addison-Wesley Publishing Company, Inc., 1988.

M. Abramowitz and I. Stegum, Handbook of mathematical functions, Dover Publications, Inc., New York. 


\section{VITA}

\section{Pierre R, Emeric}

Born in Fort-de-France, Martinique, France, August 4, 1967. Graduated from the Lycée Albert $1^{\mathrm{er}}$ in Monaco, June 1985. Obtained a Mechanical Engineering degree from ENSAM, Paris, France, June 1990 and a Ph.D. in Applied Science from The College of William and Mary in Virginia, May 1995. 